\title{
A general fictitious domain method with immersed jumps and multilevel nested structured meshes
}

\author{
Isabelle Ramière ${ }^{\mathrm{a}, \mathrm{b}, *}$, Philippe Angot ${ }^{\mathrm{a}}$, Michel Belliard ${ }^{\mathrm{b}}$ \\ ${ }^{a}$ Université de Provence and LATP-CMI, Technopôle de Château-Gombert, 13453 \\ Marseille cedex 13, France. [angot,ramiere]@cmi.univ-mrs.fr \\ ${ }^{\mathrm{b}}$ CEA-Cadarache, DEN/DTN/SMTM/LMTR, 13108 St-Paul-Lez-Durance, \\ France.[michel.belliard,isabelle.ramiere]@cea.fr
}

\begin{abstract}
This study addresses a new fictitious domain method for elliptic problems in order to handle general and eventually mixed embedded boundary conditions (E.B.C.) : Robin, Neumann and Dirichlet conditions on an immersed interface. The main interest of this fictitious domain method is to use simple structured meshes, possibly uniform Cartesian nested grids, which do not generally fit the interface but define an approximate one. A cell-centered finite volume scheme with a non-conforming structured mesh is derived to solve the set of equations with additional algebraic transmission conditions linking both flux and solution jumps through the immersed approximate interface. Hence, a local correction is devised to take account of the relative surface ratios in each control volume for the Robin or Neumann boundary condition. Then, the numerical scheme conserves the first-order accuracy with respect to the mesh step. This opens the way to combine the E.B.C. method with a multilevel mesh refinement solver to increase the precision in the vicinity of the interface. Such a fictitious domain method is very efficient: the $L^{2}$ and $L^{\infty}$-norm errors vary like $\mathcal{O}\left(h_{l^{\star}}\right)$ where $h_{l^{\star}}$ is the grid step of the finest refinement level around the interface until the residual first-order discretization error of the non-refined zone is reached.

The numerical results reported here for convection-diffusion problems with Dirichlet, Robin and mixed (Dirichlet and Robin) boundary conditions confirm the expected accuracy as well as the performances of the present method.
\end{abstract}

Key words: Fictitious domain method, embedded boundary conditions, elliptic problems, cell-centered finite volume, non-conforming structured meshes, multilevel local mesh refinement.

* Corresponding author.

Email address: ramiere@cmi.univ-mrs.fr (Isabelle Ramière). 


\section{Introduction and motivation}

This paper is devoted to the numerical validation of a new fictitious domain method for general embedded boundary conditions (E.B.C.) associated with a multilevel local mesh refinement. To compute the solution $\tilde{u}$ of a given problem $(\tilde{\mathcal{P}})$ in a domain $\tilde{\Omega}$, a fictitious domain methodology $[1,2]$ generally consists in several steps.

i) The domain $\tilde{\Omega}$ is embedded inside an auxiliary fictitious domain $\Omega$, generally larger and simpler such that : $\Omega=\tilde{\Omega} \cup \Sigma \cup \Omega_{e}$, where $\Omega_{e}$ stands for an external domain and $\Sigma$ the common interface between the two sub-domains. The domain $\Omega$ is chosen simply shaped, geometrically more simple than $\tilde{\Omega}$, see Fig. 1 .

ii) A fictitious problem $(\mathcal{P})$ is designed and solved all over the fictitious domain $\Omega$, with appropriate transmission conditions on $\Sigma$ and data in $\Omega_{e}$ in order to handle the original boundary conditions of $(\tilde{\mathcal{P}})$ on the immersed interface $\Sigma$. The restriction of the problem $(\mathcal{P})$ over the domain $\tilde{\Omega}$ is then chosen of the same type of $(\tilde{\mathcal{P}})$.

iii) The solution $\tilde{u}$ of the original problem $(\tilde{\mathcal{P}})$ is finally found as $: \tilde{u}=\left.u\right|_{\tilde{\Omega}}$, the restriction of the fictitious solution $u$ of $(\mathcal{P})$ over $\tilde{\Omega}$, or at least $\left.u\right|_{\tilde{\Omega}} \approx \tilde{u}$.

The objective is to use a simple structured mesh in $\Omega$, e.g. a uniform Cartesian grid, independent of the shape of the immersed interface $\Sigma$. This significantly simplifies and reduces the cost of the mesh generation in comparison to the use of boundary conforming structured or even unstructured meshes, specially in 3-D or for a moving interface $\Sigma$. The efficient mesh generation for the latter situations remains actually an extremely challenging problem. Moreover, the solution process of the fictitious problem $(\mathcal{P})$ with such a regular structured grid on $\Omega$ can be carried out with a fast solver.

In this work, the fictitious domain method uses a recent fracture model proposed in [3], which links both flux and solution jumps on an immersed interface $\Sigma$. The fictitious domain formulation for general E.B.C. is analyzed in [4]. The addressed problems are general second-order elliptic problems or associated parabolic ones in $\tilde{\Omega}$ with usual boundary conditions on $\Sigma$ : Dirichlet, Neumann or Fourier-Robin. An interesting feature of our approach is to allow the treatment of mixed immersed boundary conditions on $\Sigma$, i.e. the immersed interface $\Sigma$ may be the union of some parts, each of them supporting a different boundary condition. Moreover, the embedding configurations can be very general (see Fig. 1). In particular, the original domain does not need to be totally immersed inside the fictitious domain.

In practical computations, the immersed interface $\Sigma$ (which is not generally aligned with the grid lines) is roughly approximated by some $\Sigma_{h}$ lying on sides of control volumes. For example, with a uniform Cartesian grid of mesh step 
$h, \Sigma_{h}$ is a set of stair steps, see Fig. 9 . This defines an approximation $\tilde{\Omega}_{h}$ of the original domain $\tilde{\Omega}$ such that we may have $:\left|\operatorname{meas}\left(\tilde{\Omega}_{h}\right)-\operatorname{meas}(\tilde{\Omega})\right|=\mathcal{O}(h)$. This method is fast and has all the advantages of using a regular structured grid; however, the expected accuracy is only of first-order in the $L^{2}$-norm.

An adaptive local mesh refinement in the vicinity of the immersed interface $\Sigma$ is then required to increase the precision of the solution. A multigrid [5] process enables the solutions of each level to be each others connected. A FIC-like solver [6,7] is implemented by using a sequence of local nested grids having finer and finer sizes, the solution at each level being corrected by a flux residual calculated with the next finer level. The expected accuracy now varies asymptotically like $\mathcal{O}\left(h_{l^{\star}}\right)$, where $h_{l^{\star}}$ is the mesh step of the local finest grids around $\Sigma$ (last level of refinement), until the residual first-order discretization error of the non-refined zone is reached. Such a fictitious domain method combined with an adaptive multilevel local mesh refinement solver proves to work efficiently and it yields a significant improvement in the ratio of the obtained precision over the resulting cost or CPU (central processing unit) time, see also $[8,7]$ and the references therein.

A lot of papers have been now dedicated to embedded Dirichlet boundary conditions with several approaches, e.g. $[9,10,11,12,13,8,14,15]$ and the references therein, among the methods which deal with a "sharp interface". However, only few studies are devoted to other embedded boundary conditions like Neumann $[16,17,18,19,20]$ or especially Robin (or Fourier) ones [21,22,12,23,24]. Moreover, the present method does not require the use of Lagrange multipliers and hence the solution of a saddle-point problem involving the inf-sup condition to be satisfied by the discrete spaces $[11,25,26]$. Compared to the Grid Embedded Method (e.g. $[27,14,28,20]$ ), the main advantage of our fictitious model is to conserve the same local stencil of discretization to evaluate the numerical fluxes, even near the immersed interface. As in the Immersed Interface Method (see [29] for an overview), appropriate coefficients are introduced in the scheme to take into account the immersed jumps. Moreover, the algebraic transmission conditions used in the present method for the immersed jumps are more general than for the IIM where the jumps are supposed to be given, and no additional unknown is introduced near the immersed interface.

Let us mention that some fictitious domain methods use a "diffuse interface" approach to handle immersed boundary conditions, e.g. [30,31,32] and the references therein. To the best of our knowledge, only [33,32] focus on Robin boundary conditions. An interesting feature of the approach described in [32] is to combine such a fictitious domain method with multilevel local mesh refinement, while the fictitious boundary method presented in [34,35] deals with multigrid iterative filtering and special grid deformation techniques.

The structure of this paper is as follows. In Section 2, we recall the fictitious domain model with immersed jumps for an elliptic problem and we detail how 
to impose each kind of general E.B.C. Section 3 will be dedicated to the finite volume scheme devoted to compute this fictitious domain method. Then, in Section 4, an adapted refinement algorithm based on a multilevel flux restriction is introduced to increase the precision of the solution. Finally, in Section 5 some numerical results focused either on diffusion or on convection-diffusion problems illustrate the performance of the method.

\section{Fictitious domain method with immersed jumps}

Our objective is to solve, with a fictitious domain method, the following problem originally defined in a bounded domain $\tilde{\Omega} \subset \mathbb{R}^{d}$ with either a Dirichlet, a Neumann or a Robin boundary condition (or mixed) on $\partial \tilde{\Omega}=\partial \tilde{\Omega}_{D} \cup \partial \tilde{\Omega}_{R}$ : For $\tilde{\mathbf{a}} \in L^{\infty}(\tilde{\Omega})^{d \times d}, \tilde{\mathbf{v}} \in L^{\infty}(\tilde{\Omega})^{d}, \tilde{b} \in L^{\infty}(\tilde{\Omega})$ and $\tilde{f} \in L^{2}(\tilde{\Omega})$, find $\tilde{u} \in H^{1}(\tilde{\Omega})$ such that

$$
(\tilde{\mathcal{P}})\left\{\begin{aligned}
\operatorname{div}(-\tilde{\mathbf{a}} \nabla \tilde{u}+\tilde{\mathbf{v}} \tilde{u})+\tilde{b} \tilde{u} & =\tilde{f} & & \text { in } \tilde{\Omega} \\
\tilde{u} & =u_{D} & & \text { on } \partial \tilde{\Omega}_{D} \\
-(\tilde{\mathbf{a}} \nabla \tilde{u}) \cdot \mathbf{n} & =\alpha_{R} \tilde{u}+g_{R} & & \text { on } \partial \tilde{\Omega}_{R}
\end{aligned}\right.
$$

where $\mathbf{n}$ is the outward unit normal vector on $\partial \tilde{\Omega}_{R}, u_{D} \in H^{1 / 2}\left(\partial \tilde{\Omega}_{D}\right)$, $0 \leqslant \alpha_{R} \in L^{\infty}\left(\partial \tilde{\Omega}_{R}\right)$ and $g_{R} \in L^{2}\left(\partial \tilde{\Omega}_{R}\right)$.

The tensor of diffusion $\tilde{\mathbf{a}} \equiv\left(\tilde{a}_{i j}\right)_{1 \leqslant i, j \leqslant d}$ and the reaction coefficient $\tilde{b}$ verify the classical ellipticity assumptions:

$$
\begin{aligned}
& \exists a_{0}>0, \forall \boldsymbol{\xi} \in \mathbb{R}^{d}, \tilde{\mathbf{a}}(x) \boldsymbol{\xi} . \boldsymbol{\xi} \geqslant a_{0}|\boldsymbol{\xi}|^{2} \quad \text { a.e. in } \tilde{\Omega}, \\
& \text { where }|.| \text { is the Euclidean norm in } \mathbb{R}^{d}, \\
& \exists b_{0} \geqslant 0, \tilde{b}(x) \geqslant b_{0} \quad \text { a.e. in } \tilde{\Omega} \text {. }
\end{aligned}
$$

In a fictitious domain approach, the original domain $\tilde{\Omega}$ is embedded inside an open bounded polygonal domain $\Omega \subset \mathbb{R}^{d}$ such that $\Omega=\tilde{\Omega} \cup \Sigma \cup \Omega_{e}$, where $\Omega_{e}$ is the external fictitious domain and $\Sigma$ the common interface between $\tilde{\Omega}$ and $\Omega_{e}$ (see Figure 1 and 8). This interface $\Sigma \subset \mathbb{R}^{d-1}$ is called immersed interface. The fictitious domain $\Omega$ is chosen to be geometrically simple (rectangular for example in 2-D). The boundary of $\tilde{\Omega}$ is defined by $\partial \tilde{\Omega}=\tilde{\Gamma} \cup \Sigma$, and the boundary of $\Omega$ by $\partial \Omega=\tilde{\Gamma} \cup \Gamma_{e}$ (see Fig. 1 ).

Let us first notice that writing the Robin boundary condition (3) on $\Sigma$ in a fictitious domain method with $\Omega \supset \tilde{\Omega}$ is not directly possible unless accepting flux or solution jumps on $\Sigma$ or both. This problem is thus "extended" in the following manner to the whole fictitious domain $\Omega$ by using the formulation for general embedded boundary conditions introduced in [4]. 


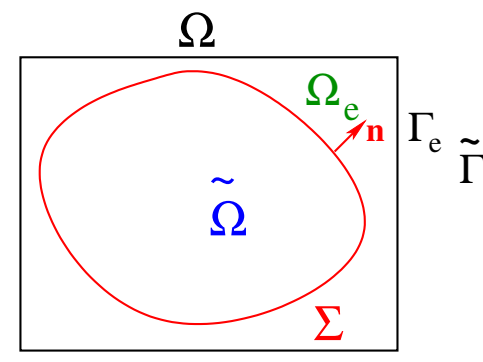

(a)

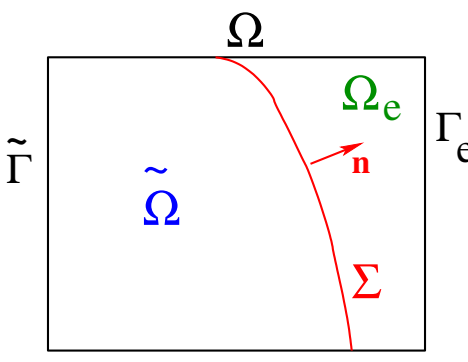

(b)

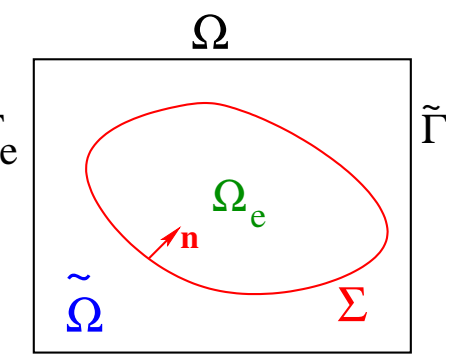

(c)

Figure 1. Embedding the original domain $\tilde{\Omega}$ inside a fictitious rectangular domain $\Omega$.

\subsection{Model with embedded jump transmission conditions on $\Sigma$}

Let $\mathbf{n}$ be either the outward unit normal vector on $\Gamma_{e}$, or the unit normal vector on $\Sigma$ oriented from $\tilde{\Omega}$ to $\Omega_{e}$. For a function $\psi$ in $H^{1}\left(\tilde{\Omega} \cup \Omega_{e}\right)$, let $\psi_{\Sigma}^{-}$and $\psi_{\Sigma}^{+}$ be the traces of $\left.\psi\right|_{\tilde{\Omega}}$ and $\left.\psi\right|_{\Omega_{e}}$ on each side of $\Sigma$ respectively, $\left.\bar{\psi}\right|_{\Sigma}=\left(\psi_{\Sigma}^{+}+\psi_{\Sigma}^{-}\right) / 2$ the arithmetic mean of traces of $\psi$, and $\llbracket \psi \rrbracket_{\Sigma}=\left(\psi_{\Sigma}^{+}-\psi_{\Sigma}^{-}\right)$the jump of traces of $\psi$ on $\Sigma$ oriented by $\mathbf{n}$.

For the data $f \in L^{2}(\Omega), g$ and $q$ given in $L^{2}(\Sigma)$, we consider the second-order elliptic problem for the real-valued function $u$ defined in $\Omega$ and including $i m$ mersed transmission conditions on $\Sigma$ which assume jumps of both the normal diffusive flux $\varphi(u) . \mathbf{n} \equiv-(\mathbf{a} \nabla u) . \mathbf{n}$ and the traces of the solution $u$ through the interface $\Sigma$ :

$(\mathcal{P})$

$$
\begin{aligned}
\operatorname{div}(-\mathbf{a} \nabla u+\mathbf{v} u)+b u=f & \text { in } \Omega, \\
\text { original B.C. } & \text { on } \tilde{\Gamma}, \\
\text { suitable B.C. } & \text { on } \Gamma_{e}, \\
\frac{\llbracket(\mathbf{a} \nabla u) \cdot \mathbf{n} \rrbracket_{\Sigma}=\left.\alpha \bar{u}\right|_{\Sigma}-q}{} & \text { on } \Sigma, \\
\left.(\mathbf{a} \nabla u) \cdot \mathbf{n}\right|_{\Sigma}=\beta \llbracket u \rrbracket_{\Sigma}-g & \text { on } \Sigma,
\end{aligned}
$$

where the uniformly positive definite tensor of diffusion $\mathbf{a} \equiv\left(a_{i j}\right)_{1 \leqslant i, j \leqslant d}$, the reaction coefficient $b \geqslant 0$, and the transfer coefficients $\alpha, \beta \geqslant 0$ on $\Sigma$ are measurable and bounded functions verifying classical ellipticity assumptions. Moreover, we choose

$$
\left.\mathbf{a}\right|_{\tilde{\Omega}}=\tilde{\mathbf{a}},\left.\quad \mathbf{v}\right|_{\tilde{\Omega}}=\tilde{\mathbf{v}},\left.\quad b\right|_{\tilde{\Omega}}=\tilde{b},\left.\quad f\right|_{\tilde{\Omega}}=\tilde{f}
$$

in order to recover the original problem (1-3) in $\tilde{\Omega}$. The B.C. (6) on $\Gamma_{e}$ is chosen to ensure the solvability of the above fictitious domain model. This problem is proved to be well-posed in $[3,4]$ and the solution $u$ belongs to $H^{1}\left(\tilde{\Omega} \cup \Omega_{e}\right)$. When $\alpha=g=q=0$ and $\beta \rightarrow \infty$, the perfect transmission problem is recov- 
ered with $u \in H^{1}(\Omega)$, see Theorem 3.2 in [12].

The data $\mathbf{a}, \mathbf{v}, b, f$ in $\Omega_{e}$ and $\alpha, \beta, g, q$ on $\Sigma$ will be defined further in order to get $\left.u\right|_{\tilde{\Omega}}=\tilde{u}$ or at least $\left.u_{\eta}\right|_{\tilde{\Omega}} \approx \tilde{u}$ when the model is penalized with a penalty parameter $\eta \rightarrow 0$.

\subsection{Treatment of the original E.B.C. on $\Sigma$}

\subsubsection{Embedded Robin or Neumann boundary conditions}

Let $\varphi_{\Sigma}^{-} \equiv-(\mathbf{a} \nabla u)^{-} .\left.\mathbf{n}\right|_{\Sigma}$ and $\varphi_{\Sigma}^{+} \equiv-(\mathbf{a} \nabla u)^{+} .\left.\mathbf{n}\right|_{\Sigma}$ be the traces of the diffusive normal flux on each side of $\Sigma$ respectively. The algebraic transmission conditions (7-8) write

$$
\begin{aligned}
\varphi_{\Sigma}^{-}-\varphi_{\Sigma}^{+} & =\frac{\alpha}{2}\left(u_{\Sigma}^{+}+u_{\Sigma}^{-}\right)-q, \\
-\frac{1}{2}\left(\varphi_{\Sigma}^{+}+\varphi_{\Sigma}^{-}\right) & =\beta\left(u_{\Sigma}^{+}-u_{\Sigma}^{-}\right)-g .
\end{aligned}
$$

The four unknown quantities $\varphi_{\Sigma}^{-}, \varphi_{\Sigma}^{+}, u_{\Sigma}^{-}$and $u_{\Sigma}^{+}$on $\Sigma$ are then linked with the algebraic transmission conditions (9-10). Hence, by eliminating one of the two exterior quantities, either $\varphi_{\Sigma}^{+}$or $u_{\Sigma}^{+}, \varphi_{\Sigma}^{-}$can be written in the two following equivalent ways, respectively :

$$
\begin{aligned}
& \varphi_{\Sigma}^{-}=\left(\beta+\frac{\alpha}{4}\right) u_{\Sigma}^{-}-\left(\beta-\frac{\alpha}{4}\right) u_{\Sigma}^{+}+g-\frac{q}{2}, \quad \text { on } \Sigma, \\
& \varphi_{\Sigma}^{-}=\frac{1}{\left(\beta+\frac{\alpha}{4}\right)}\left[\alpha \beta u_{\Sigma}^{-}+\left(\beta-\frac{\alpha}{4}\right) \varphi_{\Sigma}^{+}+\frac{\alpha}{2} g-\beta q\right], \quad \text { on } \Sigma \text {. }
\end{aligned}
$$

Note that Eq. 12 is a priori defined if $\alpha$ and $\beta$ are different from 0.

The particular choice $\beta=\alpha / 4$ requires no exterior control since both Eqs. (11) and (12) yield the same Robin boundary condition below, independently of $u_{\Sigma}^{+}$or $\varphi_{\Sigma}^{+}$:

$$
\varphi_{\Sigma}^{-} \equiv-\left.(\mathbf{a} \nabla u)^{-} \cdot \mathbf{n}\right|_{\Sigma}=\frac{\alpha}{2} u_{\Sigma}^{-}+g-\frac{q}{2}, \quad \text { on } \Sigma .
$$

When the Robin boundary condition in (3) is desired for the original problem (1-3) in $\tilde{\Omega}$, the following immersed boundary condition on $\Sigma$ must be satisfied by the solution of the fictitious domain problem in $\Omega$ :

$$
\varphi_{\Sigma}^{-} \equiv-\left.(\mathbf{a} \nabla u)^{-} \cdot \mathbf{n}\right|_{\Sigma}=\alpha_{R} u_{\Sigma}^{-}+g_{R} \quad \text { on } \Sigma \text {. }
$$

This gives the sufficient conditions for the data such that Eq. (13) satisfies (14), see (R) in the Table 1. 
The Neumann boundary condition is obtained as the particular Robin case where $\alpha_{R}=0$.

This Robin or Neumann E.B.C. method is proved to be consistent, i.e. $\left.u\right|_{\tilde{\Omega}}=\tilde{u}$, in [4]. Other variants are proposed there which require either $u_{\Sigma}^{+}$or $\varphi_{\Sigma}^{+}$to be "controlled" by $L^{2}$ or $H^{1}$ volume penalty methods $[12,13,36]$ performed with the parameters a, $b$ and $f$ in $\Omega_{e}$.

\subsubsection{Embedded Dirichlet boundary condition}

Let $0<\eta \ll 1$ be a real penalty parameter (called penalty coefficient) which is likely to tend to zero. When the Dirichlet condition $u_{\Sigma}^{-}=u_{D}$ is to be handled, the Robin condition in Eq. (3) or (14) can be penalized by a surface penalty on $\Sigma$ with $\alpha_{R}=\frac{1}{\eta}\left(\alpha_{R} \rightarrow+\infty\right)$ and $g_{R}=-\frac{1}{\eta} u_{D}$. The previous Robin E.B.C. method can be then penalized as in (D1) in the Table 1 . Hence for $g=0$ and $\beta \rightarrow+\infty$, the $(D 1)$ E.B.C method yields no solution jump $\llbracket u_{\eta} \rrbracket_{\Sigma} \rightarrow 0$ independently on $\alpha$. Thus, we can use for example the parameters : $\alpha=\beta=\frac{1}{\eta}$, $q=\frac{1}{\eta} u_{D}$ and $g=0$.

Another type of method consists in using a $L^{2}$ or $H^{1}$ volume penalty in the exterior domain $\Omega_{e}$ such that $\lim _{\eta \rightarrow 0} u_{\eta \Sigma}^{+}=u_{D}$ for the model with $\beta=\frac{1}{\eta} \rightarrow \infty$, $g=0$ and thus $\llbracket u_{\eta} \rrbracket_{\Sigma_{\eta \rightarrow 0} \rightarrow 0} 0$, as proposed in [12]. In that case, we consider an extension $u_{e} \in H^{1}\left(\Omega_{e}\right)$ of $u_{D}$ over $\Omega_{e}$ such that the trace $\left.\gamma_{0}\left(u_{e}\right) \equiv u_{e}\right|_{\Sigma}=u_{D}$, see (D2) in the Table 1.

The convergence of these methods is proved in [4] and for usual weak regularity assumptions the following error estimate : $\left\|\left.u_{\eta}\right|_{\tilde{\Omega}}-\tilde{u}\right\|_{H^{1}(\tilde{\Omega})}=\mathcal{O}(\sqrt{\eta})$ is at least reached. Better estimates are possible with stronger regularity.

Since the immersed boundary conditions are treated within the same unified formulation with appropriate data (see Table 1), it is straightforward to deal with mixed embedded boundary conditions on the immersed interface $\Sigma$. An example is given in Section 5.4. 


\begin{tabular}{|c|c|c|}
\hline E.B.C. method & Parameters in $\Omega_{e}$ & Parameters on $\Sigma$ \\
\hline \hline Robin (R) & $\left.\mathbf{a}\right|_{\Omega_{e}}=\mathbf{I d},\left.\mathbf{v}\right|_{\Omega_{e}}=\mathbf{0}$, & $\alpha=4 \beta=2 \alpha_{R}$, \\
No exterior control & $\left.b\right|_{\Omega_{e}}=\left.f\right|_{\Omega_{e}}=0$ & $g-\frac{q}{2}=g_{R}$ \\
\hline \hline Dirichlet (D1) & $\left.\mathbf{a}\right|_{\Omega_{e}}=\mathbf{I d},\left.\mathbf{v}\right|_{\Omega_{e}}=\mathbf{0}$, & $\alpha=4 \beta=\frac{2}{\eta}$, \\
Surface penalty & $\left.b\right|_{\Omega_{e}}=\left.f\right|_{\Omega_{e}}=0$ & $\frac{q}{2}-g=\frac{1}{\eta} u_{D}$ \\
\hline Dirichlet (D2) & $\left.\mathbf{a}\right|_{\Omega_{e}}=\left\{\begin{array}{c}\mathbf{I d} \quad L^{2} \text { penalty },\left.\mathbf{v}\right|_{\Omega_{e}}=\mathbf{0}, \\
\frac{1}{\eta} \mathbf{I d} H^{1} \text { penalty } \\
\left.b\right|_{\Omega_{e}}=\frac{1}{\eta},\left.f\right|_{\Omega_{e}}=\frac{1}{\eta} u_{e}\end{array}\right.$ & $\alpha=\frac{1}{\eta}$, \\
Volume penalty & \multicolumn{2}{|}{$\mid l=g=0$} \\
\hline
\end{tabular}

Table 1

Parameters in $\Omega_{e}$ and on $\Sigma$ for the E.B.C. method with immersed jumps.

\section{Finite volume (FV) discretization}

\subsection{Notations}

The polygonal domain $\Omega$ is meshed with a family $\mathcal{T}_{h}=\{K\}$ of disjointed finite volumes $K \subset \Omega$ (called "control volumes") which are open convex polygons such that $\bar{\Omega}=\underset{K \in \mathcal{T}_{h}}{\cup} \bar{K}$ with usual assumptions, e.g. [37]. The mesh size is defined by $h=\sup \left\{\operatorname{diam}(K), K \in \mathcal{T}_{h}\right\}$.

Let $\mathcal{E}$ be the family of sides (also called edges in 2-D) of the control volumes. For any $(K, L) \in \mathcal{T}_{h}^{2}$ with $K \neq L$, either the $(d-1)$-dimensional Lebesgue measure of $\bar{K} \cap \bar{L}$ is 0 or $\bar{K} \cap \bar{L}=\bar{\sigma}$ for some $\sigma \in \mathcal{E}$, which will then be denoted by $\sigma \equiv K \mid L$. The set of neighbors of $K$ is denoted by $\mathcal{N}(K)$, that is $\mathcal{N}(K)=\left\{L \in \mathcal{T}_{h} ; \exists \sigma \in \mathcal{E}, \bar{\sigma}=\bar{K} \cap \bar{L}\right\}$. For each $K \in \mathcal{T}_{h}$, let $\mathcal{E}_{K}$ be the subset of $\mathcal{E}$ such that $\partial K=\bar{K} \backslash K=\underset{\sigma \in \mathcal{E}_{K}}{\cup} \bar{\sigma}$. Furthermore, $\mathcal{E}=\underset{K \in \mathcal{T}_{h}}{\cup} \mathcal{E}_{K}$. The set of interior (resp. boundary) sides is denoting by $\mathcal{E}_{\text {int }}$ (resp. $\mathcal{E}_{\text {ext }}$ ), that is $\mathcal{E}_{\text {int }}=\{\sigma \in \mathcal{E} ; \sigma \not \subset \partial \Omega\}$ (resp. $\mathcal{E}_{\text {ext }}=\{\sigma \in \mathcal{E} ; \sigma \subset \partial \Omega\}$ ). One of the most interest of the E.B.C. method is to use a simple non-conforming and structured mesh. Therefore, the sides of the mesh $\mathcal{T}_{h}$ do not generally match the immersed interface $\Sigma$ (e.g. with a uniform Cartesian mesh like in Section 5). An approximate interface $\Sigma_{h}$ is thus defined by the mesh (see Section 5). This interface separates the approximate original domain $\tilde{\Omega}_{h}$ from the approximate external domain $\Omega_{e, h}$. Let $\mathcal{E}_{\Sigma}=\left\{\sigma \in \mathcal{E} ; \sigma \subset \Sigma_{h}\right\}$ be the set of sides lying on $\Sigma_{h}$. For each $K \in \mathcal{T}_{h}$, a "cell-centered" discretization point $x_{K} \in K$ is chosen such that for each $\sigma \in \mathcal{E}_{K} ; \sigma=K \mid L$, the segment $\left[x_{K}, x_{L}\right]$ is orthogonal to $\sigma$ at the point $x_{\sigma} \in \sigma$. For each $K \in \mathcal{T}_{h}$, let $d_{K, \sigma}=\left|x_{K}-x_{\sigma}\right|>0$ be the distance from $x_{K}$ to $\sigma$ and $\mathbf{n}_{K, \sigma}$ be the unit outward normal vector on $\sigma$. For $\sigma=K \mid L$, we then denote by $d_{\sigma}$ the Euclidean distance between $x_{K}$ 
and $x_{L}$, hence we have $d_{\sigma}=d_{K, \sigma}+d_{L, \sigma}$.

For each $K \in \mathcal{T}_{h}$ or $\sigma \in \mathcal{E}, m(K)=\int_{K} \mathrm{~d} x$ and $m(\sigma)=\int_{\sigma} \mathrm{d} s$ denote the measure of $K$ or $\sigma$. For a given quantity $\psi$, the discrete values $\psi_{K}$ and $\psi_{\sigma}$ are defined by the mean values of $\psi$ over $K$ or $\sigma$, respectively:

$$
\psi_{K}=\frac{1}{m(K)} \int_{K} \psi(x) \mathrm{d} x, \quad \text { and } \quad \psi_{\sigma}=\frac{1}{m(\sigma)} \int_{\sigma} \psi(s) \mathrm{d} s .
$$

\subsection{A numerical scheme for the immersed jumps model}

For sake of simplicity, the numerical scheme is now written for an isotropic diffusion tensor $\mathbf{a}=a(x) \mathbf{I d}$. For each $K \in \mathcal{T}_{h}$ the main discrete unknown denoted by $u_{K}$ is an approximation of the value $u\left(x_{K}\right)$ of the exact solution $u$ at the nodal point $x_{K}$ of the mesh. Two auxiliary unknowns $\left(u_{K, \sigma}, u_{L, \sigma}\right)$ are introduced on $\sigma=K \mid L \in \mathcal{E}_{\text {int }}$ which are approximations of respectively $\left.u\left(x_{\sigma}\right)\right|_{K}$ and $\left.u\left(x_{\sigma}\right)\right|_{L}$.

The cell-centered FV numerical scheme uses the scheme proposed in [3] for diffusion-reaction problems. The numerical convective flux is obtained with a monotone upwind scheme. This scheme is of first order which is in agreement with the expected accuracy of our method. This FV scheme allows jumps of diffusive and convective fluxes as well as jumps of the solution on each side $\sigma \in \mathcal{E}_{\text {int }}$ and it reads in the following synthetic form:

$$
\sum_{\sigma \in \mathcal{E}_{K}} m(\sigma)\left(F_{K, \sigma}+v_{K, \sigma} u_{K, \sigma}^{U p}\right)+m(K) b_{K} u_{K}=m(K) f_{K}, \quad \forall K \in \mathcal{T}_{h}
$$

where the numerical diffusive flux $F_{K, \sigma}$ reads for all $K \in \mathcal{T}_{h}$ :

$$
F_{K, \sigma}= \begin{cases}-a_{K} \frac{u_{K, \sigma}-u_{K}}{d_{K, \sigma}} & \text { if } \sigma=K \mid L \in \mathcal{E}_{\text {int }} \\ -a_{K} \frac{u_{\sigma}-u_{K}}{d_{K, \sigma}} & \text { if } \sigma \in \mathcal{E}_{\text {ext }} \text { with a Dirichlet B.C. on } \sigma: u=g_{D} \\ \frac{a_{K}}{a_{K}+\kappa_{\sigma} d_{K, \sigma}}\left(\kappa_{\sigma} u_{K}+\phi_{\sigma}\right) & \text { if } \sigma \in \mathcal{E}_{\text {ext }} \text { with a Robin B.C. on } \sigma:-(\mathbf{a} \cdot \nabla u) \cdot \mathbf{n}=\kappa u+\phi\end{cases}
$$

and the numerical convective flux $V_{K, \sigma}=v_{K, \sigma} u_{K, \sigma}^{U p}$ is obtained with an upstream scheme by:

$$
v_{K, \sigma}=\frac{1}{m(\sigma)} \int_{\sigma} \mathbf{v}_{K, \sigma}^{-} \cdot \mathbf{n}_{K, \sigma} \mathrm{ds}
$$


and

$$
u_{K, \sigma}^{U p}= \begin{cases}u_{K} & \text { if } v_{K, \sigma} \geqslant 0 \\ u_{\sigma} & \text { if } v_{K, \sigma}<0 \text { and } \sigma \in \mathcal{E}_{\text {ext }} \\ u_{K, \sigma} & \text { if } v_{K, \sigma}<0 \text { and } \sigma \in \mathcal{E}_{\Sigma} \cap \mathcal{E}_{\text {int }} \\ u_{L} & \text { if } v_{K, \sigma}<0 \text { and } \sigma \notin\left(\mathcal{E}_{\Sigma} \cup \mathcal{E}_{\text {ext }}\right)\end{cases}
$$

The key point of this scheme is to use transmissions conditions on all $\sigma \in \mathcal{E}_{\text {int }}$ in order to have the same generic numerical scheme for all the mesh. Thus the discretization of Eqs. (7-8) for all $\sigma=K \mid L \in \mathcal{E}_{\text {int }}$ yields respectively:

$$
\begin{aligned}
F_{K, \sigma}+F_{L, \sigma} & =\alpha_{\sigma} \frac{u_{K, \sigma}+u_{L, \sigma}}{2}-q_{\sigma} \\
\frac{1}{2}\left(F_{L, \sigma}-F_{K, \sigma}\right) & =\beta_{\sigma}\left(u_{L, \sigma}-u_{K, \sigma}\right)-\mathbf{n} . \mathbf{n}_{K, \sigma} g_{\sigma}
\end{aligned}
$$

where $\mathbf{n}$ now stands for the unit normal on $\Sigma_{h}$.

Hence, for all $\sigma=K \mid L \in \mathcal{E}_{\text {int }}$, Eqs. (16) and (17-18) enable the interface unknown $u_{K, \sigma}$ to be expressed with respect to the main discrete unknowns $\left(u_{K}\right)_{K \in \mathcal{T}_{h}}$ :

$$
\begin{aligned}
u_{K, \sigma}= & \frac{\hat{a}_{\sigma} d_{L, \sigma}}{a_{L} d_{\sigma}}\left(1+\frac{\alpha_{\sigma}}{4 \beta_{\sigma}}\right) u_{K}+\frac{\hat{a}_{\sigma} d_{K, \sigma}}{a_{K} d_{\sigma}}\left(1-\frac{\alpha_{\sigma}}{4 \beta_{\sigma}}\right) u_{L}+\frac{\hat{a}_{\sigma}}{d_{\sigma} \beta_{\sigma}} u_{K} \\
& +\frac{\hat{a}_{\sigma} d_{K, \sigma} q_{\sigma}}{a_{K} d_{\sigma}}\left(\frac{d_{L, \sigma}}{a_{L}}+\frac{1}{2 \beta_{\sigma}}\right)-\mathbf{n} \cdot \mathbf{n}_{K, \sigma} \frac{\hat{a}_{\sigma} d_{K, \sigma} g_{\sigma}}{a_{K} d_{\sigma} \beta_{\sigma}}\left(1+\frac{\alpha_{\sigma} d_{L, \sigma}}{2 a_{L}}\right)
\end{aligned}
$$

where

$$
\begin{aligned}
\hat{a}_{\sigma} & =\frac{a_{\sigma}}{1+\frac{a_{\sigma}}{\beta_{\sigma} d_{\sigma}}+\alpha_{\sigma}\left(\frac{1}{4 \beta_{\sigma}}+\lambda_{\sigma}\right)}, \quad a_{\sigma}=\frac{d_{\sigma} a_{K} a_{L}}{d_{L, \sigma} a_{K}+d_{K, \sigma} a_{L}}, \\
\lambda_{\sigma} & =\frac{d_{K, \sigma} d_{L, \sigma}}{d_{L, \sigma} a_{K}+d_{K, \sigma} a_{L}} .
\end{aligned}
$$

Therefore, the interface unknowns $u_{K, \sigma}, u_{L, \sigma}$ can also be eliminated in the diffusive flux expression as reported in [3] and we have:

$$
\begin{aligned}
F_{K, \sigma}= & -\hat{a}_{\sigma}\left(\frac{u_{L}-u_{K}}{d_{\sigma}}\right)+\frac{\alpha_{\sigma} \hat{a}_{\sigma}}{2 \beta_{\sigma} d_{\sigma}}\left(\frac{u_{K}+u_{L}}{2}\right)+\frac{\alpha_{\sigma} \hat{a}_{\sigma} d_{L, \sigma}}{a_{L} d_{\sigma}} u_{K} \\
& -\frac{\hat{a}_{\sigma} q_{\sigma}}{d_{\sigma}}\left(\frac{d_{L, \sigma}}{a_{L}}+\frac{1}{2 \beta_{\sigma}}\right)+\mathbf{n} . \mathbf{n}_{K, \sigma} \frac{\hat{a}_{\sigma} g_{\sigma}}{d_{\sigma} \beta_{\sigma}}\left(1+\frac{\alpha_{\sigma} d_{L, \sigma}}{2 a_{L}}\right),
\end{aligned}
$$


The cell-centered FV scheme (15) then requires the standard $(2 \mathrm{~d}+1)$-points stencil only. This numerical scheme is as cheap as the standard scheme without any jump. The convergence properties of the present scheme are also presented in [3].

On the sides $\sigma \in \mathcal{E}_{\text {int }} ; \sigma \notin \mathcal{E}_{\Sigma}$, we set the discrete transfer coefficients of Eqs. (17-18) in order to respect the local conservativity and the solution continuity properties :

$$
F_{K, \sigma}=-F_{L, \sigma}, \quad \text { and } \quad u_{K, \sigma}=u_{L, \sigma}, \quad \text { if } \sigma=K \mid L \in \mathcal{E}_{\text {int }} \backslash \mathcal{E}_{\Sigma}
$$

Hence,

$$
\alpha_{\sigma}=q_{\sigma}=g_{\sigma}=0 \quad \text { and } \quad \beta_{\sigma}=\frac{1}{\eta} \rightarrow \infty \quad \forall \sigma \in \mathcal{E}_{\text {int }} \backslash \mathcal{E}_{\Sigma}
$$

Otherwise, $\forall \sigma \in \mathcal{E}_{\Sigma} \cap \mathcal{E}_{\text {int }}$, the discrete transfer coefficients are equal to the mean value on $\sigma$ of the transfer coefficients of the fictitious problem $(\mathcal{P})$ lying on $\Sigma_{h}$ (see Table 1).

REMARK 1. $\forall \sigma \in \mathcal{E}_{\text {int }} ; \sigma \notin \mathcal{E}_{\Sigma}$, the local conservativity of the convective flux is also ensured

$$
V_{K, \sigma}=-V_{L, \sigma}
$$

REMARK 2. In the following numerical results, a Cartesian mesh is used. In this case, if the diffusion tensor is diagonal, the above FV scheme can be applied in each mesh direction. The non-trivial anisotropic case requires some important modifications of the FV scheme, which are beyond the scope of this paper.

\subsection{Geometric correction for a Robin E.B.C.}

When the interface $\Sigma$ is roughly approximated by the mesh into $\Sigma_{h}$, a surface correction is then required for a Robin (or a non homogeneous Neumann) embedded boundary condition. Let $\omega_{h, \Sigma}=\left\{\cup K, K \in \mathcal{T}_{h}, K \cap \Sigma \neq \emptyset\right\}$ be the union of the finite volumes crossed by $\Sigma$. Around each finite volume $K \subset \omega_{h, \Sigma}$, the local conservativity between the embedded flux integral and the approximate flux integral is ensured by

$$
\int_{\Sigma \cap \hat{K}} \varphi_{\Sigma}^{-} \mathrm{d} s=\int_{\Sigma_{h} \cap \hat{K}} \frac{1}{\epsilon_{K}} \varphi_{\Sigma_{h}}^{-} \mathrm{d} s
$$

where $\varphi_{\Sigma_{h}}^{-}=\alpha_{R} u_{\Sigma_{h}}^{-}+g_{R}, \hat{K}$ is a union of control volumes around $K$ precised below and $\epsilon_{K}$ is the local correction parameter to determine.

For all $K \subset \omega_{h, \Sigma}$, we denote by $\hat{\mathcal{E}}_{K}=\left\{\sigma \in \mathcal{E} ; \sigma \in \mathcal{E}_{K} \cap \mathcal{E}_{\Sigma}\right.$, $\sigma \in \mathcal{E}_{\text {ext }}$ or $\sigma=K \mid L$ with either $L \subset \Omega_{e, h}$ or $\left.L \not \subset \omega_{h, \Sigma}\right\}$ the set of sides lying on $\Sigma_{h}$ associated to $K$. Otherwise, for $K \not \subset \omega_{h, \Sigma}, \hat{\mathcal{E}}_{K}=\emptyset$. 
Hence, for all $\sigma \subset \Sigma_{h}, \sigma \in \hat{\mathcal{E}}_{K}$ the discrete value $\varphi_{\Sigma_{h}}^{-}$of $\varphi_{\Sigma}^{-}$and thus the discrete data $\alpha_{R}$ and $g_{R}$ on $\sigma$ are divided by the characteristic parameter $\epsilon_{K}$.

Let us now detail the calculation of $\epsilon_{K}$. This correction takes account of the relative surfaces considered in each cell $K \subset \omega_{h, \Sigma}$. For sake of simplicity, a piecewise linear approximation $\Sigma_{l}$ (see Fig. 2) of $\Sigma$ composed by a segment $\Sigma_{l, K}$ in each control volume $K \subset \omega_{h, \Sigma}$ is defined.

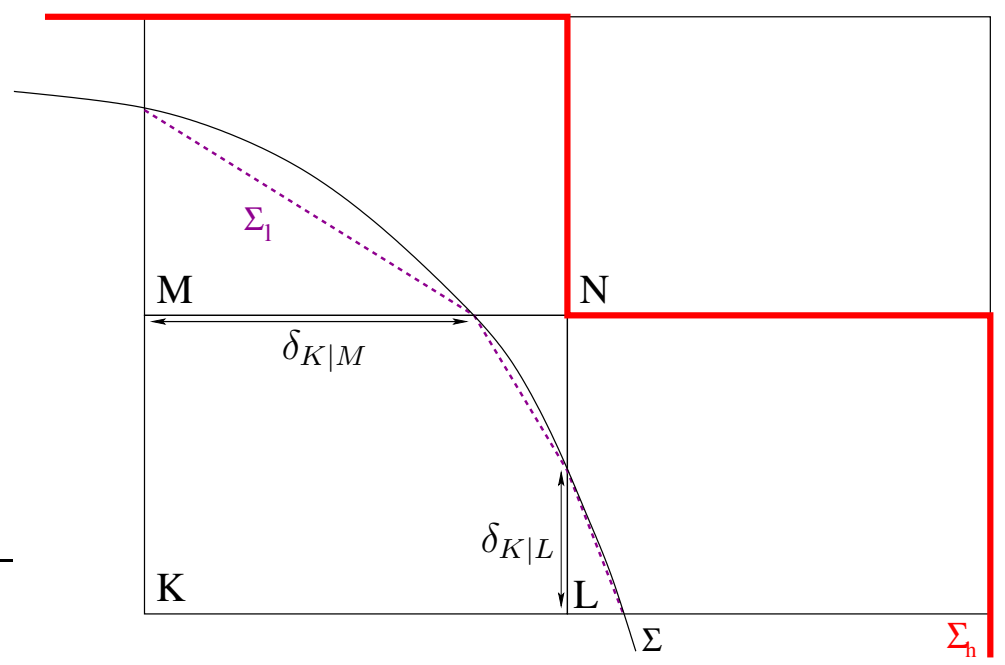

Figure 2. Local parameters in a control volume $K \subset \omega_{h, \Sigma}$.

So, a natural way to evaluate the correction parameter $\epsilon_{K}$ in each $K \subset \omega_{h, \Sigma}$ would be

$$
\begin{gathered}
\sum_{\epsilon_{K}} m(\sigma) \\
\forall \sigma \subset \hat{\mathcal{E}}_{K}\left(\Sigma_{l, K}\right) \\
\operatorname{meas}\left(\Sigma_{h}, \sigma \in \hat{\mathcal{E}}_{K}, \quad \epsilon_{\sigma}=\epsilon_{K}\right.
\end{gathered}
$$

However, for some cells $K \subset \omega_{h, \Sigma}$, we may have $\hat{\mathcal{E}}_{K}=\emptyset$, for example $K \subset \omega_{h, \Sigma}$ without any $\sigma \in \mathcal{E}_{\Sigma}$ (see Fig. 2) or $K \subset\left(\omega_{h, \Sigma} \cap \Omega_{e, h}\right)$ with $\sigma=K \mid L \in \mathcal{E}_{\Sigma}$ where $L \subset \omega_{h, \Sigma}$. For such a cell, since $\epsilon_{K}=0$, the approximate measure of the segment $\Sigma_{l, K}$ is never taken into account. Then,

$$
\begin{aligned}
\sum_{\sigma \in \mathcal{E}_{\Sigma}} \int_{\sigma} \frac{1}{\epsilon_{\sigma}} & =\sum_{\sigma \in \mathcal{E}_{\Sigma}} \frac{m(\sigma)}{\epsilon_{\sigma}} \\
& =\sum_{K \subset \omega_{h, \Sigma} ; \hat{\mathcal{E}}_{K} \neq \emptyset} \frac{1}{\epsilon_{K}} \sum_{\sigma \in \hat{\mathcal{E}}_{K}} m(\sigma) \\
& =\sum_{K \subset \omega_{h, \Sigma} ; \hat{\mathcal{E}}_{K} \neq \emptyset} \operatorname{meas}\left(\Sigma_{l, K}\right)<\operatorname{meas}\left(\Sigma_{l}\right) \approx \operatorname{meas}(\Sigma),
\end{aligned}
$$

and the conservativity of the fluxes will not be ensured. 
In order to take account of the entire measure of the immersed interface $\Sigma$, for the cells $K \subset \omega_{h, \Sigma}$ such that $\hat{\mathcal{E}}_{K}=\emptyset$, the measure of $\Sigma_{l, K}$ is added to the measure of $\Sigma_{l, K^{\prime}}$ of one of the neighbor $K^{\prime} \in \mathcal{N}(K)$ with $\hat{\mathcal{E}}_{K^{\prime}} \neq \emptyset$. The choice of the neighbor $K^{\prime}$ (called in the sequel "chosen" neighbor) can be made by different ways, for example arbitrarily among all the neighbors $L \in \mathcal{N}(K)$ such that $\hat{\mathcal{E}}_{L} \neq \emptyset$. Another choice is detailed hereafter. Let us underline that for each $K \subset \omega_{h, \Sigma}$ such that $\hat{\mathcal{E}}_{K}=\emptyset$, the "chosen" neighbor is defined uniquely in order to take account of the measure of $\Sigma_{l, K}$ only once.

For all $K \subset \omega_{h, \Sigma}$ such that $\hat{\mathcal{E}}_{K} \neq \emptyset$, let $\mathcal{C}(K)=\left\{L \in \mathcal{T}_{h} ; L \in \mathcal{N}(K), L \subset \omega_{h, \Sigma}\right.$, $\hat{\mathcal{E}}_{L}=\emptyset, K$ is the "chosen" neighbor of $\left.L\right\}$ be the set of cells of which $K$ is the "chosen" neighbor. Then, we define $\hat{\Sigma}_{l, K}$ such that:

$$
\hat{\Sigma}_{l, K}=\Sigma_{l, K}+\sum_{L \in \mathcal{C}(K)} \Sigma_{l, L}
$$

and we have

$$
\hat{K}=K \cup(\underset{L \in \mathcal{C}(K)}{\cup} L) .
$$

Thus, $\forall K \subset \omega_{h, \Sigma}$ such that $\hat{\mathcal{E}}_{K} \neq \emptyset$, the local correction parameter $\epsilon_{K}$ becomes

$$
\epsilon_{K}=\frac{\sum_{\sigma \in \hat{\mathcal{E}}_{K}} m(\sigma)}{\operatorname{meas}\left(\hat{\Sigma}_{l, K}\right)}
$$

And

$$
\forall \sigma \subset \Sigma_{h}, \sigma \in \hat{\mathcal{E}}_{K}, \quad \epsilon_{\sigma}=\epsilon_{K}
$$

In order to determine the "chosen" neighbor, we favour the neighbor cell having the most important intersection with the original domain $\tilde{\Omega}$. To do this, in each $K \subset \omega_{h, \Sigma}$ with $\hat{\mathcal{E}}_{K}=\emptyset$, and for each side $\sigma=K \mid L \in \mathcal{E}_{K} \cap \mathcal{E}_{\text {int }}$ straddling the original domain $\tilde{\Omega}$ and the exterior domain $\Omega_{e}$, we denote by $\delta_{K \mid L}$ the proportion of $\sigma$ lying in the original domain $\tilde{\Omega}$ (see Fig. 2). Among the neighbors $L \in \mathcal{N}(K)$ such that $\hat{\mathcal{E}}_{L} \neq \emptyset$, the "chosen" neighbour $K^{\prime}$ is taken such that $\delta_{K \mid K^{\prime}}=\max _{L} \delta_{K \mid L}$.

REMARK 1. The local surface ratio $\epsilon_{K}$ (see Eq.(26)) is defined within an $\mathcal{O}\left(h_{K}\right)$ error, where $h_{K}$ is the diameter of the cell $K$. For example, an approximation of $\epsilon_{K}$ when $\Sigma$ is a circle, as in the following numerical tests, can be found in [23].

REMARK 2. A global correction can be also considered with a rough estimation of $\epsilon_{K}$ by a constant value $\epsilon_{h}$ over the whole interface $\Sigma_{h}$. In this case, it is the ratio between the surfaces of $\Sigma_{h}$ and $\Sigma$ :

$$
\epsilon_{h}=\frac{\operatorname{meas}\left(\Sigma_{h}\right)}{\operatorname{meas}(\Sigma)}
$$


However, this global correction is not accurate enough to ensure the convergence of the fictitious domain algorithm with respect to the mesh step $h$ in the general case (see the numerical results in Section 5).

\section{REMARK 3. Theoretical error estimates}

We are interesting in error estimates of the discrete fictitious solution $u_{h}$ over the approximate original domain $\tilde{\Omega}_{h}$. Indeed the aim of our fictitious domain approach is to find an approximation of the exact solution $\tilde{u}$ defined in $\tilde{\Omega}$ using a simple, possibly uniform, Cartesian mesh.

In [38], a convergence study of the error is carried out for a $Q_{1}$-finite element method with Cartesian non-boundary fitted meshes for both Dirichlet and Robin boundary conditions. This analysis can be considered as a subproblem of the convergence study of the previous fictitious domain methods. In this case, the immersed boundary conditions are supposed to be perfectly imposed on the approximate interface or at least the modelling error in order to impose the immersed boundary conditions is assumed to be negligible compared to the approximation error of the immersed interface.

For a regular mesh, if the exact solution $\tilde{u}$ belongs to $H^{2}(\tilde{\Omega})$, the following estimates hold in the approximate domain $\tilde{\Omega}_{h}$ :

$$
\begin{aligned}
\left\|\tilde{u}-u_{h}\right\|_{H^{1}\left(\tilde{\Omega}_{h}\right)} & =\mathcal{O}\left(h^{1 / 2}\right), \\
\left\|\tilde{u}-u_{h}\right\|_{L^{2}\left(\tilde{\Omega}_{h}\right)} & =\mathcal{O}(h) .
\end{aligned}
$$

These estimates only depend on appropriate norms of the original data and the $H^{2}$-norm of the exact solution $\tilde{u}$ in the original domain $\tilde{\Omega}$.

For a cell-centered FV scheme as introduced in the previous section, it is natural to obtain similar error estimates. The numerical results reported in Section 5 confirm that the error of the discrete solution $u_{h}$ restricted to $\tilde{\Omega}_{h}$ is of order $h$ in the $L^{2}$ norm. Hence the estimates in the approximate domain $\tilde{\Omega}_{h}$ seem not to depend on the regularity of the fictitious problem solution over $\Omega$. Indeed, the solution of the whole fictitious problem $(\mathcal{P})$ with immersed jumps does not generally belong to $H^{1}(\Omega)$ (when $\llbracket u \rrbracket_{\Sigma} \neq 0$ ), but the convergence remains of first-order for the $L^{2}$-norm. For the Lagrange multiplier approach, in [39] the same conclusion is drawn looking to the numerical interior error (see page 501: in the interior of $\tilde{\Omega}, u_{h}$ probably satisfies local error estimates that involve only the values of $\tilde{u}$ in $\tilde{\Omega}$ ).

\section{Adaptive mesh refinement (AMR) solver}

The E.B.C. method proposed in this paper uses a structured (most often Cartesian) uniform mesh. As explained in the previous section, this mesh defines an approximate interface $\Sigma_{h}$ on which the E.B.C. are imposed. In order 
to improve the accuracy of the solution, the present fictitious domain method is combined with a local multilevel mesh refinement solver in the vicinity of the immersed interface $\Sigma$.

\subsection{Multigrid method}

A multigrid method [5] is based on a recursive process that consists in generating local subgrids $G_{l}, 0 \leqslant l$, having finer and finer discretization steps $h_{l}$ until reaching a given level $l^{\star}$. The differential problem under study is solved on each computational grid. The solutions between each level are connected through the following interactions:

- the boundary conditions on the fine grids $G_{l}, 0<l \leqslant l^{\star}$, are interpolated from the solution to the next coarser grid $G_{l-1}$ via a prolongation operator $P_{l}^{l-1}$,

- in the problem solved on the coarse grids $G_{l}, 0 \leqslant l<l^{\star}$, some corrective terms evaluated from the solution to next finer grid $G_{l+1}$, are added to the right-hand side by a restriction operator $R_{l}^{l+1}$.

Iterations of a multigrid process can be represented for example by $\mathrm{V}$-cycles (see Fig. 3).

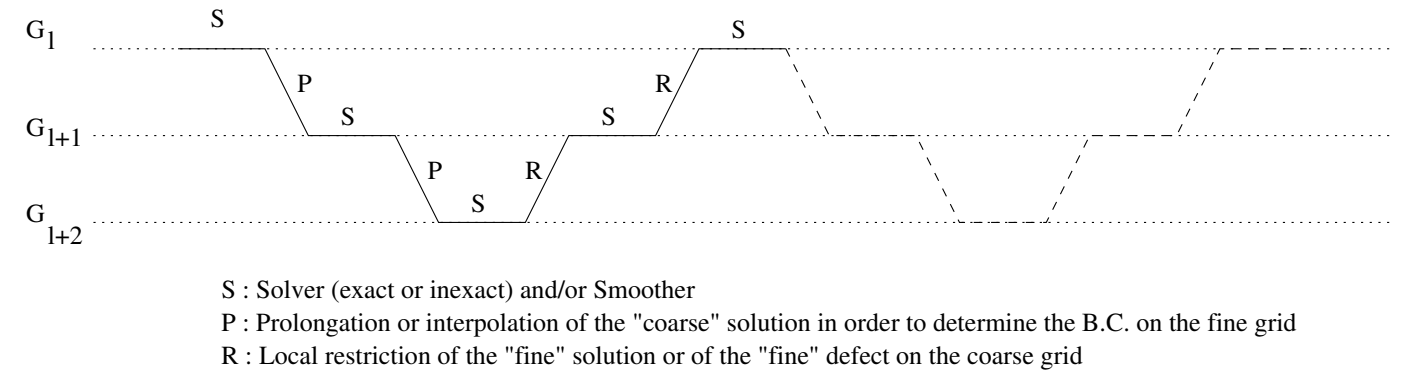

Figure 3. Multigrid process

In our approach, each level of refinement $l$ consists of a set of local patches $G_{l}$. Each patch $G_{l}$ is a nested subgrid around a single control volume at the next coarser level $l-1$.

\subsection{FIC method}

The principle of the Flux Interface Correction (FIC) $[40,41,6,7]$ method is based on the respect of a flux weak continuity relation between two grids that are non-concordant. A conservative adjustment between the nested subgrids is then ensured.

In the sequel, we use the same notations than those introduced in Section 3.1. 
Moreover the subscript $l$ refers to the level of refinement under consideration. For $0 \leqslant l<l^{\star}$, we denote by $\mathcal{A}_{l}$ the union of control volumes at the level $l$ that are included in the refinement area. The restriction operator $R_{l}^{l+1}$ is a flux restriction operator which takes part into the evaluation of a flux residual on each side of the control volume $K_{l} \subset \mathcal{A}_{l}$.

Let us consider the resolution of the following problem:

$$
(\mathcal{P})\left\{\begin{array}{l}
L u=f \text { in } \Omega \\
B . C . \quad \text { on } \Gamma=\partial \Omega
\end{array}\right.
$$

At each iteration $k$ of the FIC algorithm, the following discrete problem is solved on the subdomain $\Omega_{l}$ associated to the grid $G_{l}$ :

$$
\left(\mathcal{P}_{l}^{k}\right) \begin{cases}L_{l}^{k} u_{l}^{k}=f_{l}^{k} & \text { in } \Omega_{l} \\ \text { B.C. } & \text { on } \Gamma_{l}=\partial \Omega_{l}\end{cases}
$$

Let us assume that the operator $L$ can be divided into a conservative part (with a divergence term) involving the flux $\mathcal{F}(u)$ and a non-conservative part $\mathcal{G}(u)$ :

$$
L u=\operatorname{div}(\mathcal{F}(u))+\mathcal{G}(u)=f
$$

For each $K_{l} \subset \mathcal{A}_{l}$ on a coarse level $l, 0 \leqslant l<l^{\star}$, a local flux correction residual $r_{l}(\mathcal{F})$ is defined by

$$
r_{l}(\mathcal{F})\left(K_{l}\right)=\frac{1}{\operatorname{meas}\left(K_{l}\right)}\left\{R_{l}^{l+1}(\mathcal{F}(u))\left(K_{l}\right)-\sum_{\sigma_{l} \subset \partial K_{l}} \int_{\sigma_{l}} \mathcal{F}_{l}(u) \cdot \mathbf{n}_{K_{l}, \sigma_{l}}\right\}
$$

where $R_{l}^{l+1}$ is the flux restriction operator

$$
R_{l}^{l+1}(\mathcal{F}(u))\left(K_{l}\right)=\sum_{\left\{\sigma_{l+1} \subset \partial K_{l} ; K_{l+1} \subset K_{l}\right\}} \int_{\sigma_{l+1}} \mathcal{F}_{l+1}(u) \cdot \mathbf{n}_{K_{l+1}, \sigma_{l+1}}
$$

At each iteration $k$, a correction term is added to the right-hand side of the equation of the problem $\left(\mathcal{P}_{l}^{k}\right)$ :

$$
\left\{\begin{array}{l}
f_{l}^{0}=\left.f\right|_{\Omega_{l}} \\
f_{l}^{k}=f_{l}^{k-1}+\sum_{K_{l} \subset \mathcal{A}_{l}} \chi_{K_{l}} r_{l}^{k}(\mathcal{F})\left(K_{l}\right)
\end{array}\right.
$$

where $r_{l}^{k}(\mathcal{F})\left(K_{l}\right)$ is the flux residual (see Eq. (29)) evaluated at the iteration $k$ for $u=u^{k}$ and $\chi$ is the characteristic function. 


\subsection{A FIC-like solver for the E.B.C. method}

The FIC method is well adapted to a conservative discretization of the equations on multilevel nested meshes. As the FV scheme introduced in Section 3 is locally conservative, a FIC solver can be used with the present E.B.C. method.

\subsubsection{Rectangular local patches}

At each level $l$, a set of local nested subgrids $G_{l}$ with the same grid step $h_{l}$ are obtained from the following process:

- The original coarse grid $G_{0}$ corresponds to the mesh $\mathcal{T}_{h}$ of the whole domain $\Omega$. The mesh size $h=h_{0}$.

- At each level $l, 0 \leqslant l \leqslant l^{\star}$, the approximate interface $\Sigma_{h_{l}}$, the approximate original domain $\tilde{\Omega}_{h_{l}}$, the approximate external domain $\Omega_{e, h_{l}}$ as well as $\omega_{h_{l}, \Sigma}$ are detected (see Fig. 4(a)).

- For $0 \leqslant l<l^{\star}$, each control volume $K_{l} \subset \omega_{h_{l}, \Sigma}$ or such that $\mathcal{E}_{K_{l}} \cap\left(\mathcal{E}_{\Sigma}\right)_{l} \neq \emptyset$ generates a subgrid or patch at the level $l+1$ with the grid step $h_{l+1}<h_{l}$. The domain of this refinement patch is composed of the "parent" cell $K_{l}$ enlarged of $h_{l+1}$ on all sides (see Fig. 4(b)). By this way, the interpolated B.C. on each subgrid $G_{l+1}$ are not directly imposed on the sides of the parent cell. Naturally, if one of the sides of $K_{l}$ lies on the boundary $\partial \Omega$, there is no enlargement in this direction. In the sequel, we will denote by $G_{l}^{\text {int }}$ the set of control volumes $K_{l} \in G_{l}$ which are not in the enlargement zone of $G_{l}$. Then, let $\mathcal{A}_{l}=\left\{\cup K_{l} ; K_{l} \in G_{l}^{i n t}, K_{l} \subset \omega_{h_{l}, \Sigma}\right.$ or $\left.\mathcal{E}_{K_{l}} \cap\left(\mathcal{E}_{\Sigma}\right)_{l} \neq \emptyset\right\}$ be the refinement area on each level $l, 0 \leqslant l<l^{\star}$. As a fact, a control volume lying in the enlargement zone of $G_{l}$ does not generate a refinement patch nested in $G_{l}$, but may induce a refinement patch connected to a neighbor patch of $G_{l}$.

Since all the nested subgrids are simply shaped (rectangular in 2-D), the E.B.C. solver (see Section 2) can be easily implemented on each patch in a recursive way.

\subsubsection{A FIC-like solver}

For sake of clarity, we suppose now that $h_{l+1}=\frac{h_{l}}{2}$ in each direction. Hence, each patch $G_{l}$ has $4^{d}$ cells at most, i.e. 16 cells in 2-D and 64 cells in 3-D. 


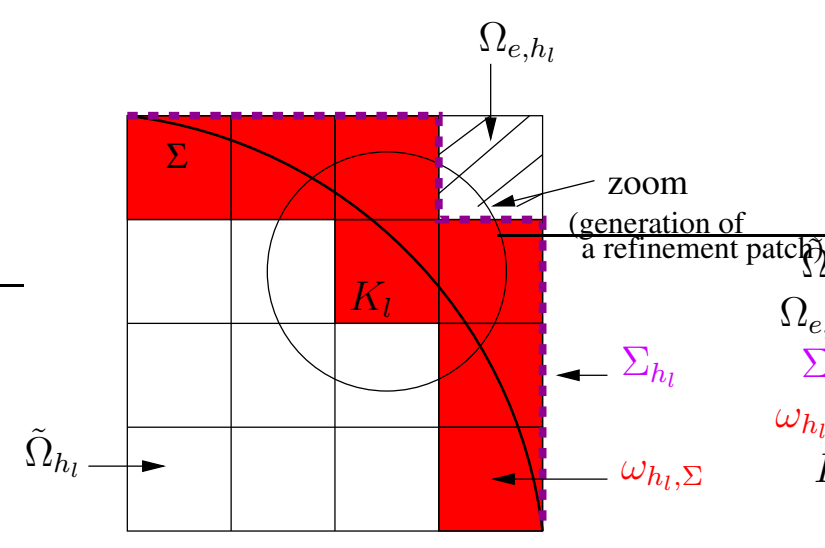

(a) Level $l$

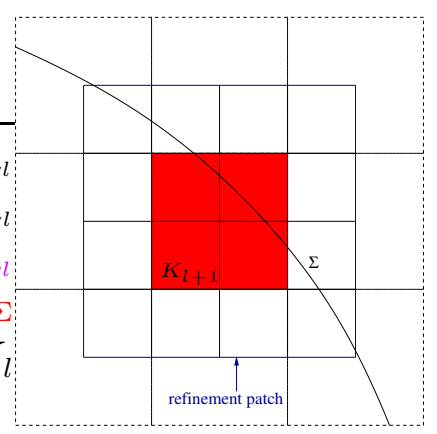

(b) Patch $G_{l+1}$ around $K_{l}$

Figure 4. Generation of the refinement patches

\subsubsection{The prolongation operator $P_{l}^{l-1}$}

The boundary conditions on $\Gamma_{l}=\partial \Omega_{l}$ are divided into two subsets:

- On $\Gamma_{l} \cap \Gamma$, the B.C. of the original problem are imposed,

- On $\Gamma_{l} \backslash\left(\Gamma_{l} \cap \Gamma\right)$, Dirichlet B.C. are imposed by a prolongation operator $P_{l}^{l-1}$.

The E.B.C. method introduced here allows jumps of flux and solution. The prolongation operator $P_{l}^{l-1}$ is then an interpolation operator which takes account of the solution jumps. As the FV scheme (see Section 3) enables $u_{K_{l-1}, \sigma_{l-1}}$ to be evaluated (see Eq. (19)), a linear interpolation is implemented from the values $\left(u_{K_{l-1}}, u_{K_{l-1}, \sigma_{l-1}}\right)_{K_{l-1} \in G_{l-1}}$.

We use the following linear interpolation formula

$$
f(a)=\frac{f(a-\delta)+f(a+\delta)}{2}
$$

along the segment $[a-\delta, a+\delta]$.

In our case, $f(a)$ represents $u_{K_{l}, \sigma_{l}}$. The interpolation nodes $\{a-\delta, a+\delta\}$ depend on the dimension $d$ of the problem. For 2-D problems, $f(a-\delta)$ and $f(a+\delta)$ represent either $u_{K_{l-1}}$ or $u_{K_{l-1}, \sigma_{l-1}}$, while for 3-D problems $f(a-\delta)$ and $f(a+\delta)$ are both side values $u_{K_{l-1}, \sigma_{l-1}}$ (with different $\sigma_{l-1} \in \mathcal{E}_{K_{l-1}}$ ), see Fig. 5 and Fig. 6.

REMARK: if $h_{l+1} \neq \frac{h_{l}}{2}$, the linear interpolation formula becomes a bilinear interpolation formula where the interpolation nodes are four of the values $u_{K_{l-1}, \sigma_{l-1}}$ of the cell $K_{l-1}$. 


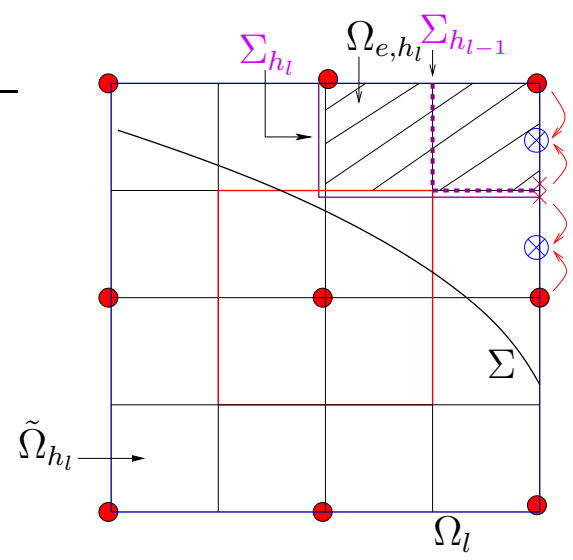

$$
\begin{aligned}
& u_{K_{l-1}} \\
\times & u_{K_{l-1}, \sigma_{l-1}} \\
\otimes & u_{K_{l}, \sigma_{l}}
\end{aligned}
$$

Figure 5. 2-D case : Nodes of interpolation for the prolongation operator $P_{l}^{l-1}$.

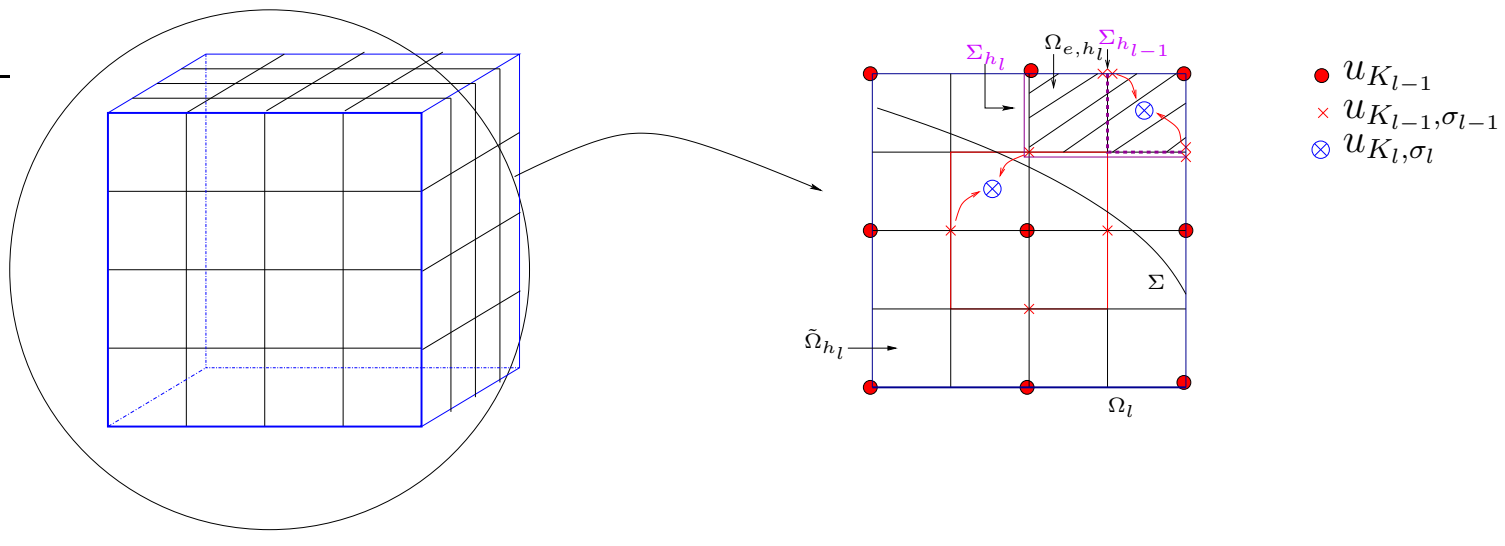

Figure 6. 3-D case : Nodes of interpolation for the prolongation operator $P_{l}^{l-1}$.

\subsubsection{The restriction operator $R_{l}^{l+1}$}

The restriction operator $R_{l}^{l+1}$ is based on the FIC restriction operator (see Eq. (30)). Then, $r_{l}(\mathcal{F})$ will take into account the flux residual between the level $l$ and the level $l+1$. With the E.B.C. method, since the approximate interface $\Sigma_{h_{l}}$ is defined on each level $l$, a control volume $K_{l} \subset \tilde{\Omega}_{h_{l}}$ in the approximate original domain can contain control volumes $K_{l+1} \subset \Omega_{e, h_{l+1}}$ in the approximated external domain (see Fig. 7). As the approximate original domain $\tilde{\Omega}_{h}$ and the external one $\Omega_{e, h}$ are uncoupled, the flux residual has to be evaluated on sides of control volumes in the approximate original domain only.

Moreover, on the sides $\sigma_{l} \in\left(\mathcal{E}_{\Sigma}\right)_{l}$, the immersed boundary conditions are imposed. These sides are then boundary sides of the approximate original domain $\tilde{\Omega}_{h_{l}}$. So, the flux correction residual $r_{l}(\mathcal{F})$ is obtained by the difference between the fluxes $\mathcal{F}_{l}(u)$ and $\mathcal{F}_{l+1}(u)$ on strictly interior sides of the approximate original domains only. Hence, at level $l$, the interior flux will be corrected on the approximate original domain. If we denote by $\left(\mathcal{E}_{r e f}\right)_{l}=\left\{\sigma_{l} \in \mathcal{E}_{l} ; \sigma_{l} \in\right.$ $\left.\left(\mathcal{E}_{\text {int }}\right)_{l} \backslash\left(\mathcal{E}_{\Sigma}\right)_{l} ; \sigma_{l} \subset \tilde{\Omega}_{h_{l}}\right\}$ the set of sides at the level $l$ which are strictly interior 


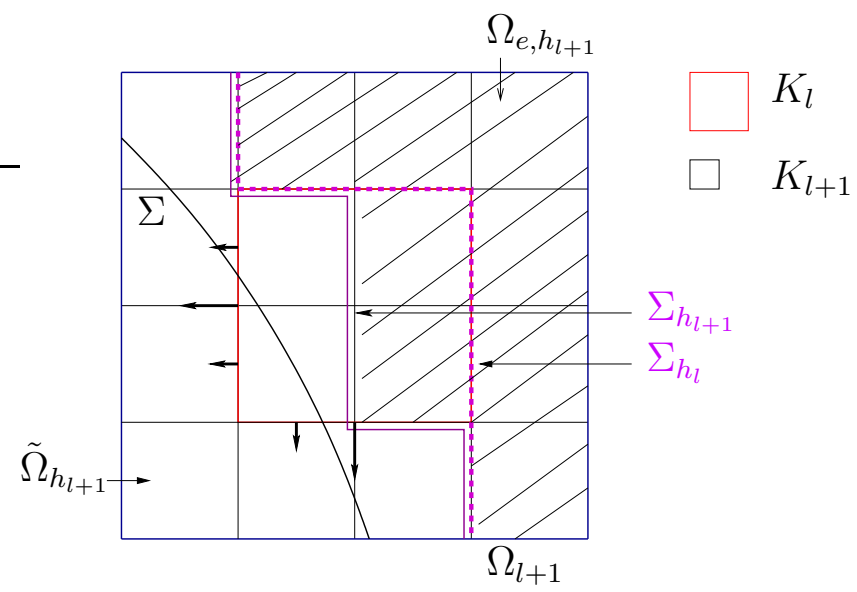

Figure 7 . Interior control volume $K_{l}$ with exterior control volumes $K_{l+1}$ to the approximate original domain $\tilde{\Omega}_{h_{l}}$, then $\forall K_{l} \subset \mathcal{A}_{l}$,

$$
r_{l}(\mathcal{F})\left(K_{l}\right)=\frac{1}{\operatorname{meas}\left(K_{l}\right)}\left\{R_{l}^{l+1}(\mathcal{F}(u))\left(K_{l}\right)-\sum_{\left\{\sigma_{l} \subset \partial K_{l} ;\right.} \int_{\sigma_{l}} \mathcal{F}_{l}(u) \cdot \mathbf{n}_{K_{l}, \sigma_{l}}\right\}
$$

with the restriction operator $R_{l}^{l+1}$ defined by

$$
\begin{aligned}
R_{l}^{l+1}(\mathcal{F}(u))\left(K_{l}\right)= & \sum_{\substack{\left\{\sigma_{l+1} \subset \partial K_{l} ; K_{l+1} \subset K_{l}\\
\\
\right.}} \int_{\sigma_{l+1} \in\left(\mathcal{E}_{r e f}\right)_{l+1}} \mathcal{F}_{l+1}(u) \cdot \mathbf{n}_{K_{l+1}, \sigma_{l+1}} \\
& \left.\sigma_{l+1} \subset \sigma_{l} \in\left(\mathcal{E}_{r e f}\right)_{l}\right\}
\end{aligned}
$$

\subsubsection{Domain decomposition method (DDM)}

Due to the patches enlargement (see Section 4.3.1), there is an overlapping between some patches of the same level. Hence, on each level $0<l \leqslant l^{\star}$ (at descent and ascent phases of the $\mathrm{V}$-cycles), we perform a small number of domain decomposition iterations (typically 3 ) via a standard multiplicative Schwarz procedure (see e.g. [42]) acting as a smoother. Moreover, at the ascent phase, in order to obtain the same solution on a control volume belonging to different patches, the same equation has to be solved on this control volume. The righthand side corresponding to a restricted control volume $K_{l} \subset \mathcal{A}_{l}, K_{l} \in G_{l}^{\text {int }}$, is then imposed as right-hand side of all the equations solved on this control volume.

For some kind of approximations of the immersed interface (see for example the "cut" interface of the Section 5), control volumes $K_{l} \subset \mathcal{A}_{l}$ belonging to the approximate external domain $\Omega_{e, h_{l}}$ at the level $l$, may contain control volumes $K_{l+1}$ included in the approximate original domain $\tilde{\Omega}_{h_{l+1}}$ at the level $l+1$. In this case, at the level $l+1$, the DDM smoothing iterations (Schwarz iterations and right-hand side correction) are really necessary to have a better approxi- 
mation of the flux $\mathcal{F}_{l+1}(u)$ on the neighbors patches and then to obtain a good evaluation of the restriction operator $R_{l}^{l+1}$.

\subsubsection{The FIC-EBC algorithm}

The multilevel FIC-EBC algorithm writes for V-cycles:

Initialization: Computation of $u_{0}^{0}$, the solution to the original problem $\left(\mathcal{P}_{0}^{0}\right)$ on the grid $G_{0}$.

Iterations: Computation of $u_{l}^{k}$.

for $k=1$ to $n b V$ cycles do

$u_{0}^{k}=u_{0}^{k-1}$.

Resolution on the fine grids $G_{l}$ :

for $l=1$ to $l^{\star}$ do

(i) Compute B.C. on $\Gamma_{l} \backslash\left(\Gamma_{l} \cap \Gamma\right)$ by interpolation of $u_{l-1}^{k}$ :

$$
u_{l}^{k}=P_{l}^{l-1} u_{l-1}^{k} \text { on } \Gamma_{l} \backslash\left(\Gamma_{l} \cap \Gamma\right)
$$

where $P_{l}^{l-1}$ is the prolongation operator.

(ii) Computation of $u_{l}^{k}$ by solving $\left(\mathcal{P}_{l}^{k}\right)$ with $f_{l}^{k}=f_{l}^{k-1}$.

(iii) DDM iterations (Schwarz iterations).

\section{endfor}

Correction on the coarse grids $G_{l}$ :

for $l=l^{\star}-1$ to 0 do

(i) Evaluation of the flux residual $r_{l}^{k}(\mathcal{F})$ defined for $\forall K_{l} \subset \mathcal{A}_{l}$ by

$$
\begin{aligned}
r_{l}^{k}(\mathcal{F})\left(K_{l}\right)=\frac{1}{\operatorname{meas}\left(K_{l}\right)}\{ & R_{l}^{l+1}\left(\mathcal{F}\left(u^{k}\right)\right)\left(K_{l}\right) \\
- & \left.\sum_{\substack{\left\{\sigma_{l} \subset \partial K_{l} ; \\
\sigma_{l} \in\left(\mathcal{E}_{\text {ref }}\right)_{l}\right\}}} \int_{\sigma_{l}} \mathcal{F}_{l}\left(u^{k}\right) \cdot \mathbf{n}_{K_{l}, \sigma_{l}}\right\}
\end{aligned}
$$

where $R_{l}^{l+1}$ is the restriction operator.

(ii) Computation of the corrected solution $u_{l}^{k}$ : resolution of the problem $\left(\mathcal{P}_{l}^{k}\right)$ with $\left\{\begin{array}{l}f_{l}^{0}=\left.f\right|_{\Omega_{l}} \\ f_{l}^{k}=f_{l}^{k-1}+\sum_{K_{l} \subset \mathcal{A}_{l}} \chi_{K_{l}} r_{l}^{k}(\mathcal{F})\left(K_{l}\right)\end{array}\right.$.

(iii) DDM iterations (Schwarz iterations and correction of the right-hand side in the enlargement zone) endfor endfor 
In the discrete case, with the notations of Section 3.1, the flux integrals are approximated by the following quadrature formula, which is in agreement with the FV discretization

$$
\forall l, 0 \leqslant l \leqslant l^{\star}, \quad \int_{\sigma_{l}} \mathcal{F}_{l}^{k}(u) \cdot \mathbf{n}_{K_{l}, \sigma_{l}}=m\left(\sigma_{l}\right)\left(\mathcal{F}_{l}^{k}(u) \cdot \mathbf{n}_{K_{l}, \sigma_{l}}\right)\left(x_{\sigma_{l}}\right)
$$

The stopping criterium for the V-cycles is chosen as $\frac{\left\|u_{0}^{k+1}-u_{0}^{k}\right\|}{\left\|u_{0}^{k}\right\|} \leqslant 10^{-4}$ in the following numerical results. The steepest refinement level tested here is $l^{\star}=3$ (i.e. 4 levels including the initial coarse level). This leads to a convergence of the algorithm within about $10 \mathrm{~V}$-cycles.

\section{$5 \quad$ Numerical results}

In what follows, we focus on 2-D problems, even though the simulation of 3-D problems is straightforward.

\subsection{Solvers}

In the computations, if we consider an isotropic diffusion tensor $\mathbf{a}=a(x) \mathbf{I d}$, the discretization of a diffusion problem leads to a symmetric positive definite linear system. A conjugate gradient iterative algorithm [43] is used to solve the linear system. However, for a convection-diffusion problem discretized with an upstream scheme for the convective flux, the linear system is no more symmetric. A Bi-GCSTAB [44] algorithm is then implemented. For all the resulting linear systems, a diagonal preconditioner is used to improve the ill-conditioning due to the penalization coefficients. Looking for a better preconditioning will be an interesting study for further works. However, the choice of a better precontionner is not so crucial since the multilevel solver involves for the local patches the resolution of linear systems with a small number of unknowns. The stopping criterium of the Krylov gradient methods is $\left\|r_{k}\right\| \leqslant 10^{-9}$, where $r_{k}$ is the residual vector at the $k^{t h}$ iteration of the algorithm. Hence, the diagonal preconditioned gradient methods converge within about 10 iterations on the local patches. 


\subsection{Discrete norms}

The errors between the numerical and analytic solutions to the following test problems can be appreciate by the calculation of either the discrete $L^{2}$-norm or the $L^{\infty}$-norm (max norm) in $\tilde{\Omega}_{h}$.

For the approximate solution $u_{h}$, the discrete $L^{2}$-norm in the approximate original domain $\tilde{\Omega}_{h}$ is defined by

$$
\left\|u_{h}\right\|_{L^{2}\left(\tilde{\Omega}_{h}\right)}=\left(\sum_{K \subset \tilde{\Omega}_{h}} \operatorname{meas}(K)\left|u_{K}\right|^{2}\right)^{1 / 2}
$$

and for the analytic solution $\tilde{u}$ to the original test problems

$$
\|\tilde{u}\|_{L^{2}\left(\tilde{\Omega}_{h}\right)}=\left(\sum_{K \subset \tilde{\Omega}_{h}} \operatorname{meas}(K)\left|\tilde{u}\left(x_{K}\right)\right|^{2}\right)^{1 / 2} .
$$

Then a relative discrete $L^{2}$-norm of the error is evaluated by

$$
e_{r}\left(u_{h}\right)=\frac{\left\|\tilde{u}-u_{h}\right\|_{L^{2}\left(\tilde{\Omega}_{h}\right)}}{\|\tilde{u}\|_{L^{2}\left(\tilde{\Omega}_{h}\right)}} .
$$

By definition, the discrete $L^{\infty}$-norm is:

$$
\left\|\tilde{u}-u_{h}\right\|_{L^{\infty}\left(\tilde{\Omega}_{h}\right)}=\max _{K \subset \tilde{\Omega}_{h}}\left|\tilde{u}\left(x_{K}\right)-u_{K}\right| .
$$

\subsection{First test problem: a quarter disk domain}

We solve a 2-D boundary value problem either for convection-diffusion or diffusion alone in a quarter of the unit disk $\tilde{\Omega}$ with symmetry conditions on the axis $\tilde{\Gamma}$ (see Fig. 8(a)). The fictitious domain is the unit square $\Omega=] 0,1[\times] 0,1$, see Fig. 8(b).

The domain $\Omega$ is meshed by uniform square cells $K$ with a grid step varying from $h=\frac{1}{4}$ to $h=\frac{1}{512}$. This defines the exterior approximate interface $\Sigma_{h}^{e x t}$ such that $\tilde{\Omega} \subset \tilde{\Omega}_{h}$ and the cut approximate interface $\Sigma_{h}^{c u t}$ which may cross the physical immersed interface $\Sigma$, see Fig. 9. The resulting approximate physical domains $\tilde{\Omega}_{h}$ are such that: $\mid$ meas $\left(\tilde{\Omega}_{h}\right)-$ meas $(\tilde{\Omega}) \mid=\mathcal{O}(h)$, or more precisely, $\operatorname{meas}\left(\left(\tilde{\Omega} \cup \tilde{\Omega}_{h}\right) \backslash\left(\tilde{\Omega} \cap \tilde{\Omega}_{h}\right)\right)=\mathcal{O}(h)$. 


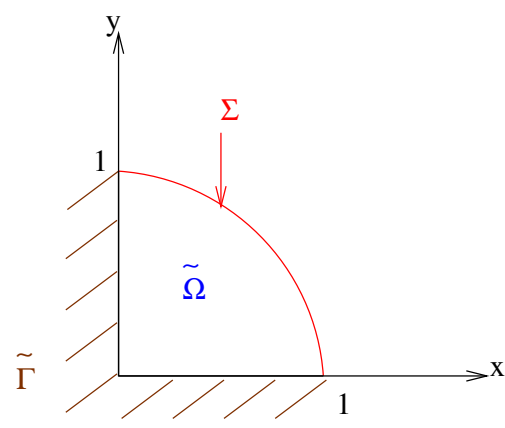

(a) Original domain $\tilde{\Omega}$.

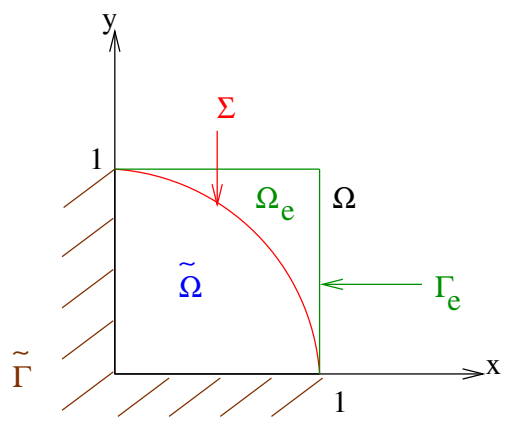

(b) Fictitious domain $\Omega$.

Figure 8. Immersion of the unit disk into the unit square.

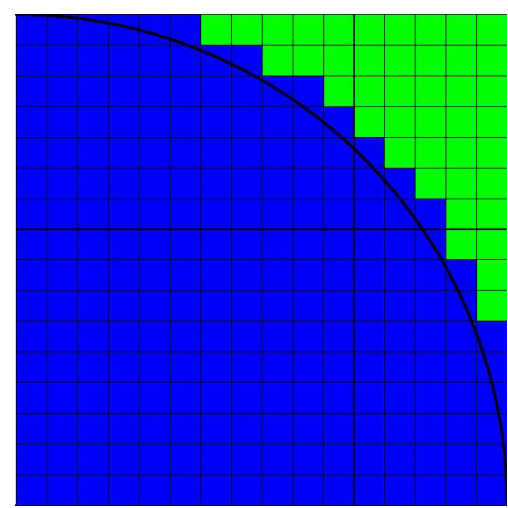

(a) Exterior approximate interface $\Sigma_{h}^{e x t}$.

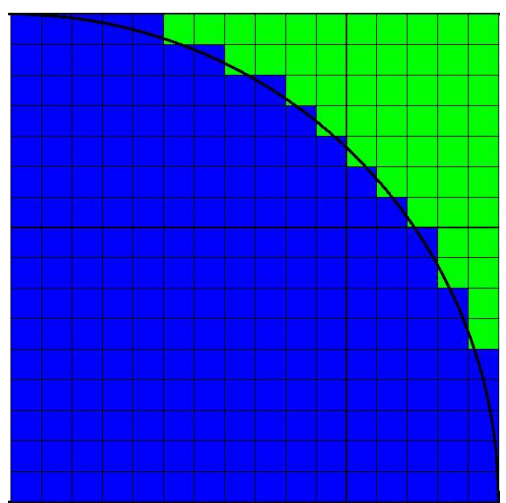

(b) Cut approximate interface $\Sigma_{h}^{c u t}$.

Figure 9. Non-conforming mesh, approximate interfaces $\Sigma_{h}$ and approximate domains $\tilde{\Omega}_{h}$.

\subsubsection{Diffusion problems}

\subsubsection{Dirichlet problem}

First, we consider the following homogeneous Dirichlet problem

$$
(\tilde{\mathcal{P}})\left\{\begin{aligned}
-\triangle \tilde{u} & =4 \text { in } \tilde{\Omega} \\
\frac{\partial \tilde{u}}{\partial n} & =0 \text { on } \tilde{\Gamma}, \\
\tilde{u} & =0 \text { on } \Sigma\left(u_{D}=0\right),
\end{aligned}\right.
$$

which has the analytic solution

$$
\tilde{u}=1-r^{2} \quad \text { in } \tilde{\Omega} \quad \text { where } \quad r=\sqrt{x^{2}+y^{2}} \text { denotes the radius in } \tilde{\Omega} \text {. }
$$


The fictitious domain problem is solved in $\Omega$ with the E.B.C. method (D1), using only the surface penalty on $\Sigma_{h}$ without exterior control, and (D2), using both a surface penalty on $\Sigma_{h}$ and a volume $H^{1}$ penalty in $\Omega_{e, h}$, described in the Table 1 of Section 2 with $u_{e} \equiv 0$.

In Fig. 10, we observe that the $L^{2}$-norm of the modelling error varies with respect to the penalization parameter $\eta$ better than $\mathcal{O}\left(\eta^{\frac{1}{2}}\right)$ for $(D 1)$, and $\mathcal{O}\left(\eta^{\frac{3}{4}}\right)$ for $(D 2)$. These results are in agreement with the $H^{1}$ estimation of the modelling error in [4]. The stagnation plateau shown in this figure corresponds to the value of the space discretization error. The following computations are performed with $\eta=10^{-12}$ to get the modelling error negligible compared to the discretization error.

\section{Relative $\mathrm{L}^{2}$ error norms versus eta}

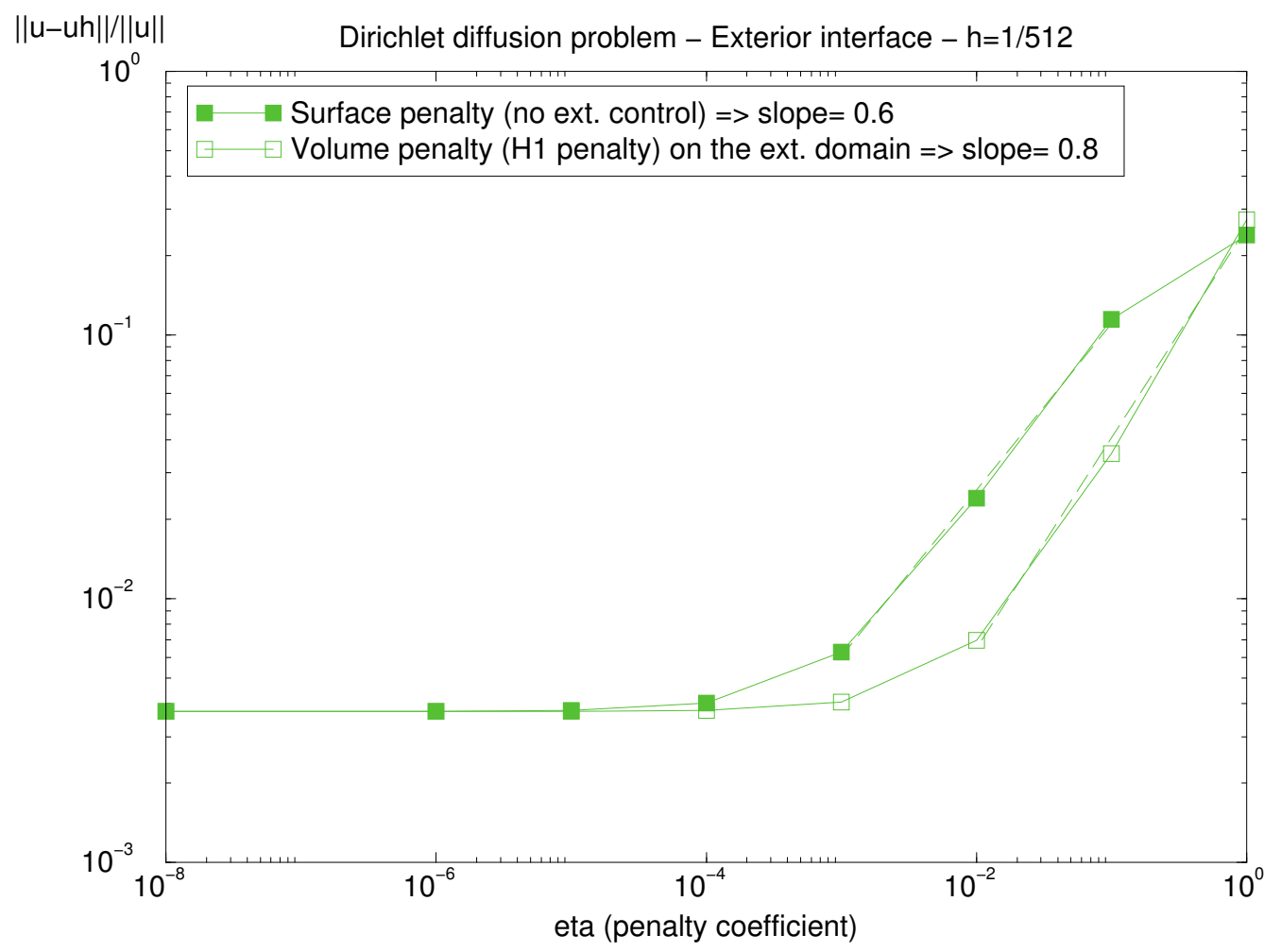

Figure 10. Convergence for the $L^{2}$-norm of the modelling error with $\eta$ in the Dirichlet case for $(D 1)$ and $(D 2)$ E.B.C. methods with $\Sigma_{h}^{e x t}$ and $h=\frac{1}{512}$.

The Fig. 11 shows the convergence of the numerical error with respect to the discretization step $h$. Both E.B.C. methods $(D 1)$ and $(D 2)$ exhibit a first-order convergence for the $L^{2}$-norm, as expected since $\left|\operatorname{meas}\left(\tilde{\Omega}_{h}\right)-\operatorname{meas}(\tilde{\Omega})\right|=\mathcal{O}(h)$ only. We can also observe that for a given discretization $\Sigma_{h}$ (either exterior or cut) of the immersed interface $\Sigma$, the two variants (D1) and (D2) lead to 
the same errors. Indeed, performing a $H^{1}$ penalty method in $\Omega_{e, h}$, the solution and its gradient are penalized. So $u_{\eta} \simeq u_{D}$ on the exterior domain $\Omega_{e, h}$ until the approximated interface $\Sigma_{h}$. As no solution jump on $\Sigma_{h}$ is also imposed by penalization, the $(D 2)$ method leads to $u_{\eta} \simeq u_{D}$ on $\Sigma_{h}$ as for the surface penalty method $(D 1)$.

Moreover, the methods which use the cut approximate interface are more accurate since $\Sigma_{h}^{c u t}$ approximates $\Sigma$ more precisely than $\Sigma_{h}^{e x t}$.

In Fig. 12(a), the error distribution $\left|\tilde{u}\left(x_{K}\right)-u_{K}\right| \forall K \subset \tilde{\Omega}_{h}$, illustrates that the main differences between the approximate solution and the analytic one are located on the grid cells crossed by the immersed interface.

Then the (D1) E.B.C. method is combined with the FIC-EBC solver (see Section 4.3), the refinement zone of which is located around the immersed interface. The two-grid algorithm converges within about $10 \mathrm{~V}$-cycles. The number of $\mathrm{V}$-cycles for the convergence almost does not increase with the number of refinement levels. The first conclusion to draw from Fig. 12(b), is that the error values are reduced when the FIC-EBC solver is applied. We can also remark some error spikes. These spikes are localized around the immersed interface, on the cells $K \subset \omega_{h, \Sigma}=\left\{\cup K, K \in \mathcal{T}_{h}, K \cap \Sigma \neq \emptyset\right\}$. Indeed, on these cells, the embedded Dirichlet boundary condition is still imposed on the sides $\sigma \subset \Sigma_{h}$, while the interior fluxes on $\sigma \subset \tilde{\Omega}_{h}$ and hence the interior solution are corrected by the FIC-EBC solver. For each cell $K \subset \omega_{h, \Sigma}$, the most important is the distance between the approximate immersed interface $\Sigma_{h}$ and the original interface $\Sigma$ (see Fig. 9), the most important is the error. The $L^{2}$ error norms obtained for several levels of refinement are reported in Fig. 13.

As shown in Fig. 13 the combination of the E.B.C. method with the FIC-EBC solver conserves the first order accuracy for the $L^{2}$-norm. Moreover, the error on the original coarse grid is similar to the error obtained without refinement on a mesh with a discretization step equal to the local finest grid's discretization step. The numerical results enable us to conclude that for the $L^{2}$-norm, the accuracy of the method varies like $\mathcal{O}\left(h_{l^{\star}}\right)$ where $h_{l^{\star}}$ is the mesh step of the local finest refinement grid (last level of refinement). However, since the error of the whole coarse mesh depends also on the error of the non-refined zone, the method varies like $\mathcal{O}\left(h_{l^{\star}}\right)$ for $l^{\star}$ less than a maximum number of refinement levels (see details in Section 5.5).

This multi-grid technique is low cost since each level of refinement contains a small number of degrees of freedom. Table 2 and Fig. 14 show that in term of CPU (central processing unit), the combination of the (D1) E.B.C. method with the FIC-EBC solver becomes interesting from a relative error lower than $5.10^{-2}$. For example, the CPU time is divided by 2 between the error obtained with $h_{0}=\frac{1}{512}$ and $l^{\star}=0$ (without refinement -1 level) and the same error obtained with $h_{0}=\frac{1}{128}$ and $l^{\star}=2$ (3 levels). Moreover, for a given error, the memory storage required is always smaller with the FIC-EBC solver. 


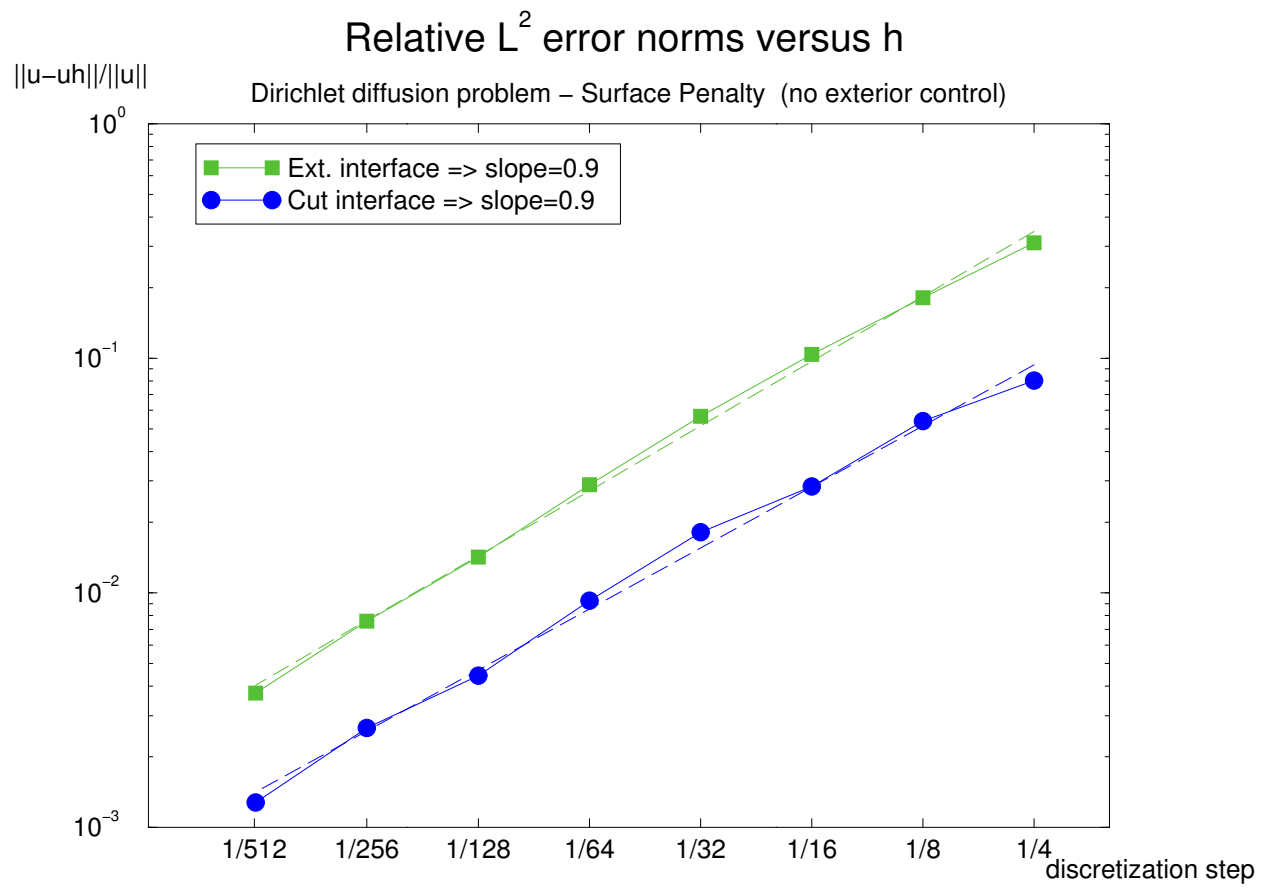

(a) (D1) E.B.C. method.

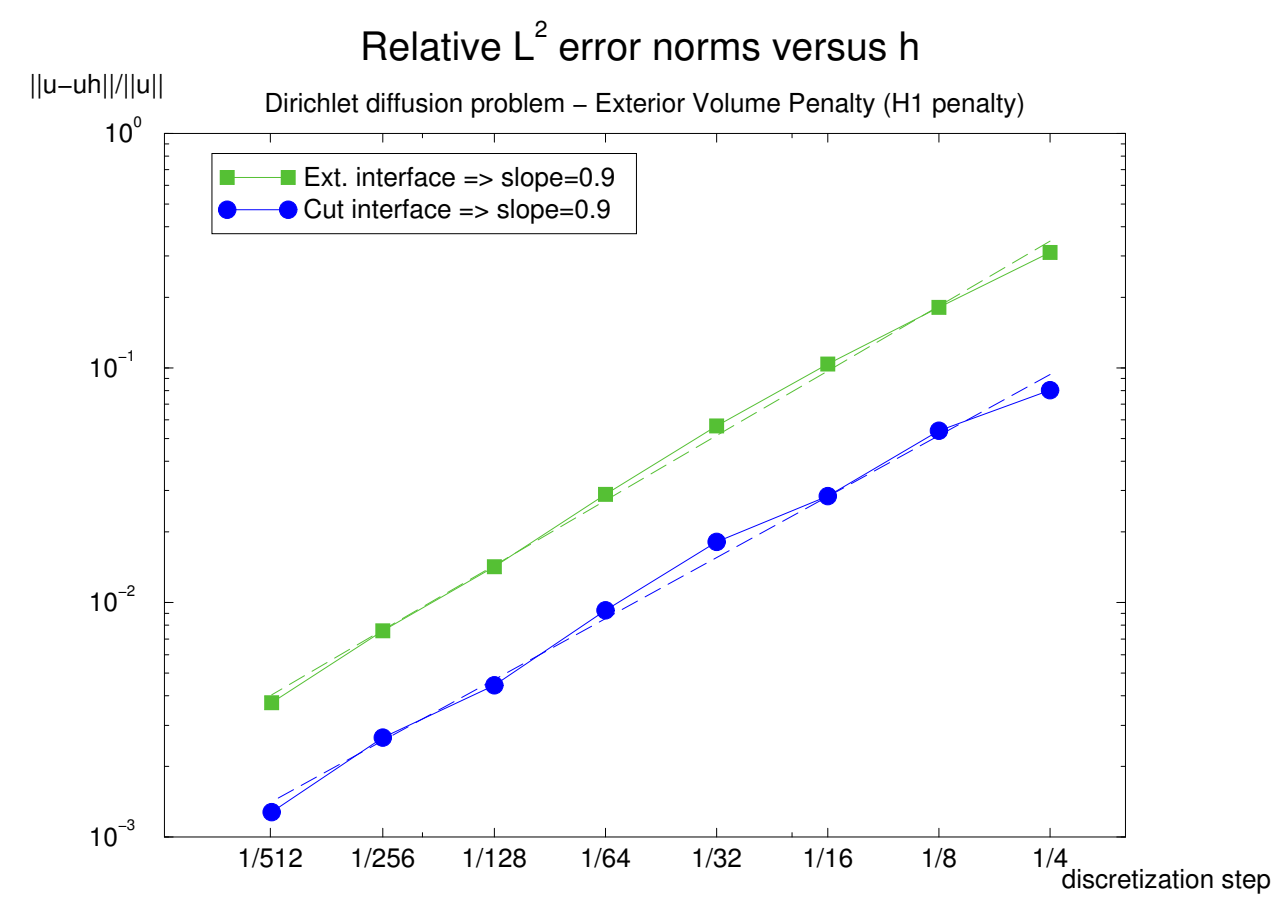

(b) (D2) E.B.C. method.

Figure 11. Convergence for the $L^{2}$-norm of the discretization error with $h$ for the Dirichlet diffusion problem with $\Sigma_{h}^{e x t}$ or $\Sigma_{h}^{c u t}$ and $\eta=10^{-12}$. 


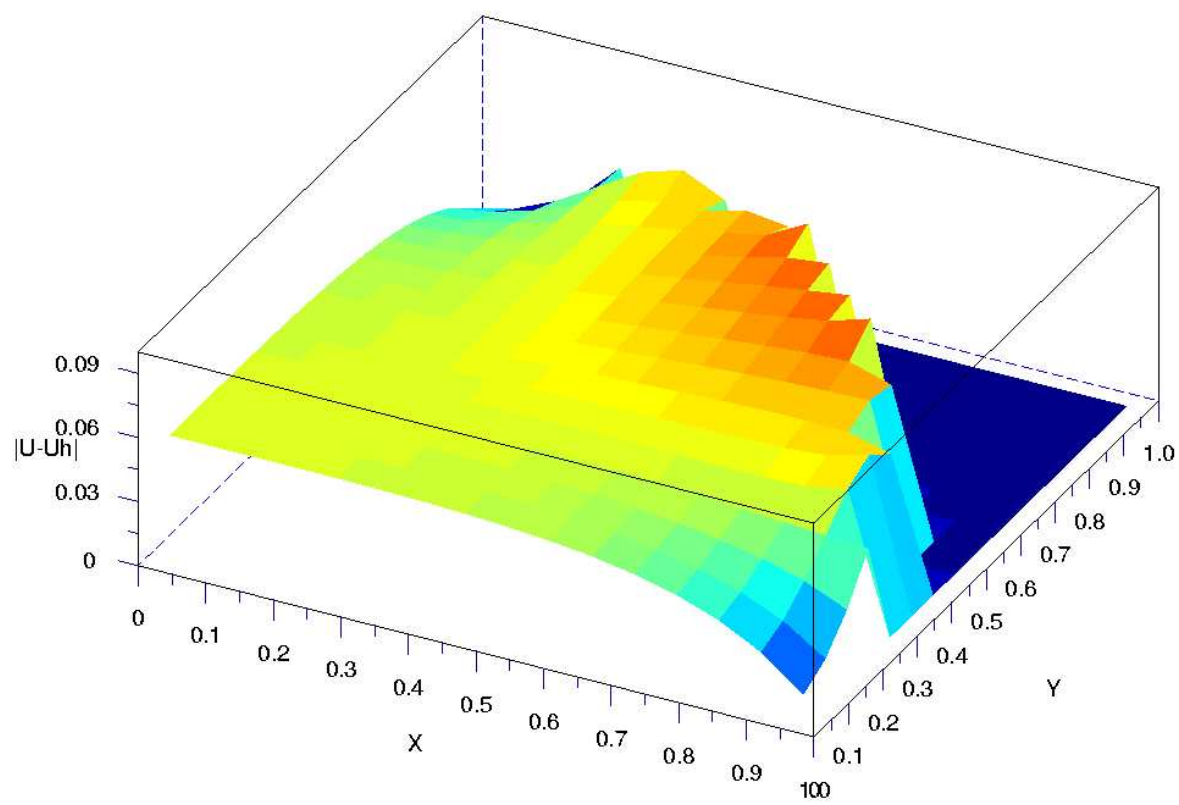

(a) Without refinement $\left(l^{\star}=0\right), \max _{K \subset \tilde{\Omega}_{h}}\left|\tilde{u}\left(x_{K}\right)-u_{K}\right|=7.910^{-2}$.

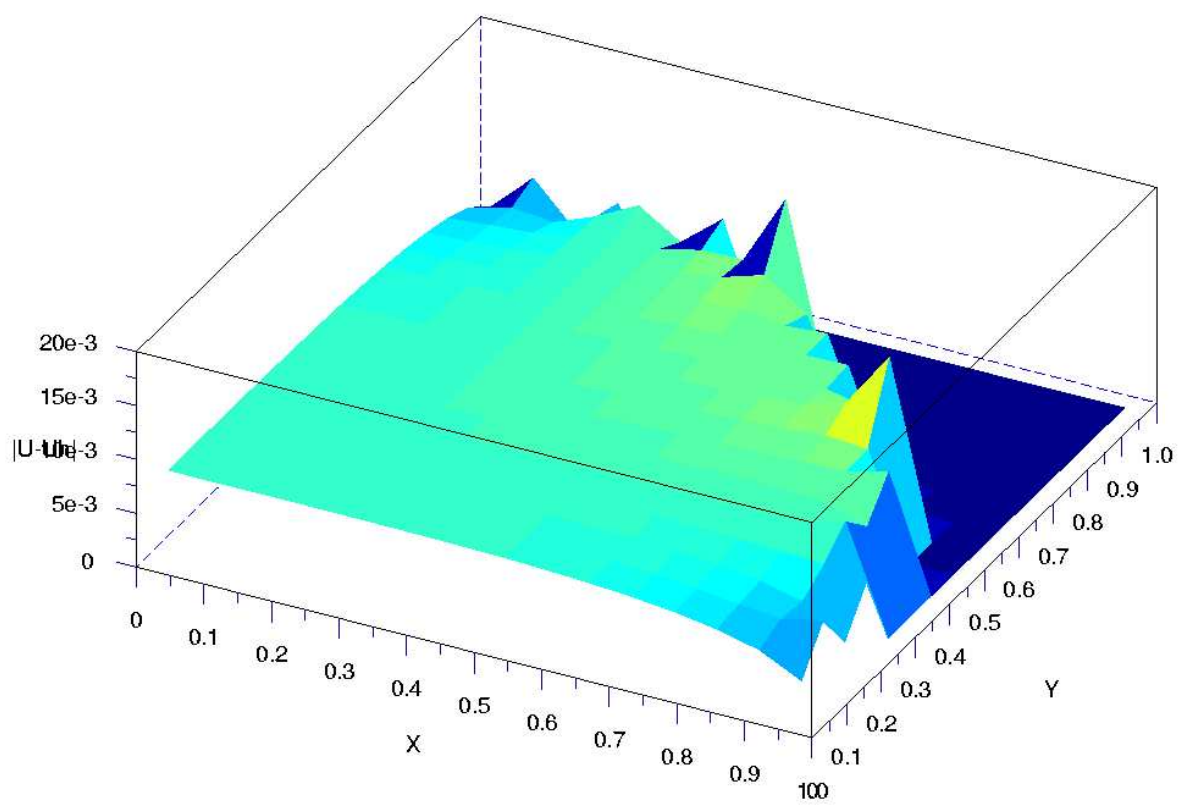

(b) With refinement: $l^{\star}=3, \max _{K \subset \tilde{\Omega}_{h}}\left|\tilde{u}\left(x_{K}\right)-u_{K}\right|=1.110^{-2}$.

Figure 12. Error distribution with a $16 \times 16$ mesh for the $(D 1)$ E.B.C. method with $\Sigma_{h}^{e x t}$ before and after the combination with the FIC-EBC solver (with DDM iterations) - Dirichlet diffusion problem. 
(D1) E.BC. method + FIC-EBC solver + DDM

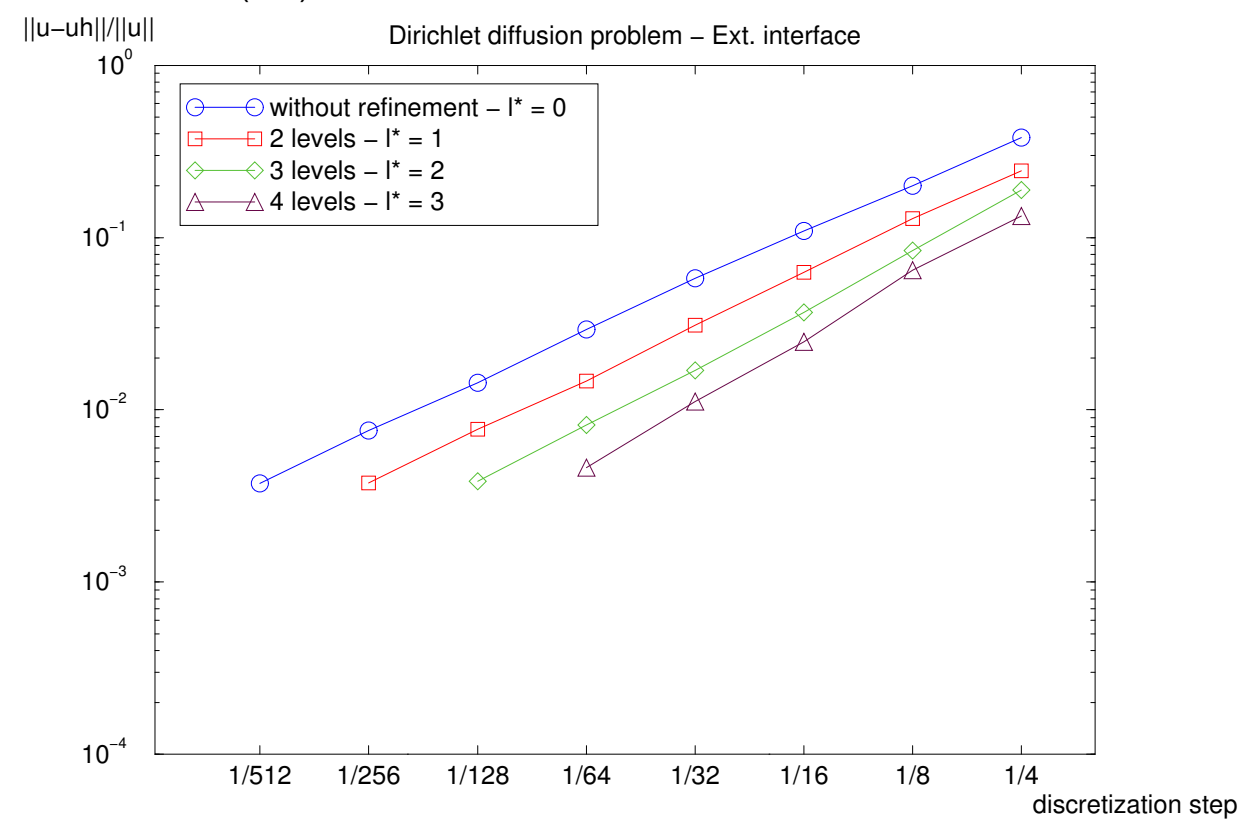

(a) Exterior interface $\Sigma_{h}^{e x t}$.

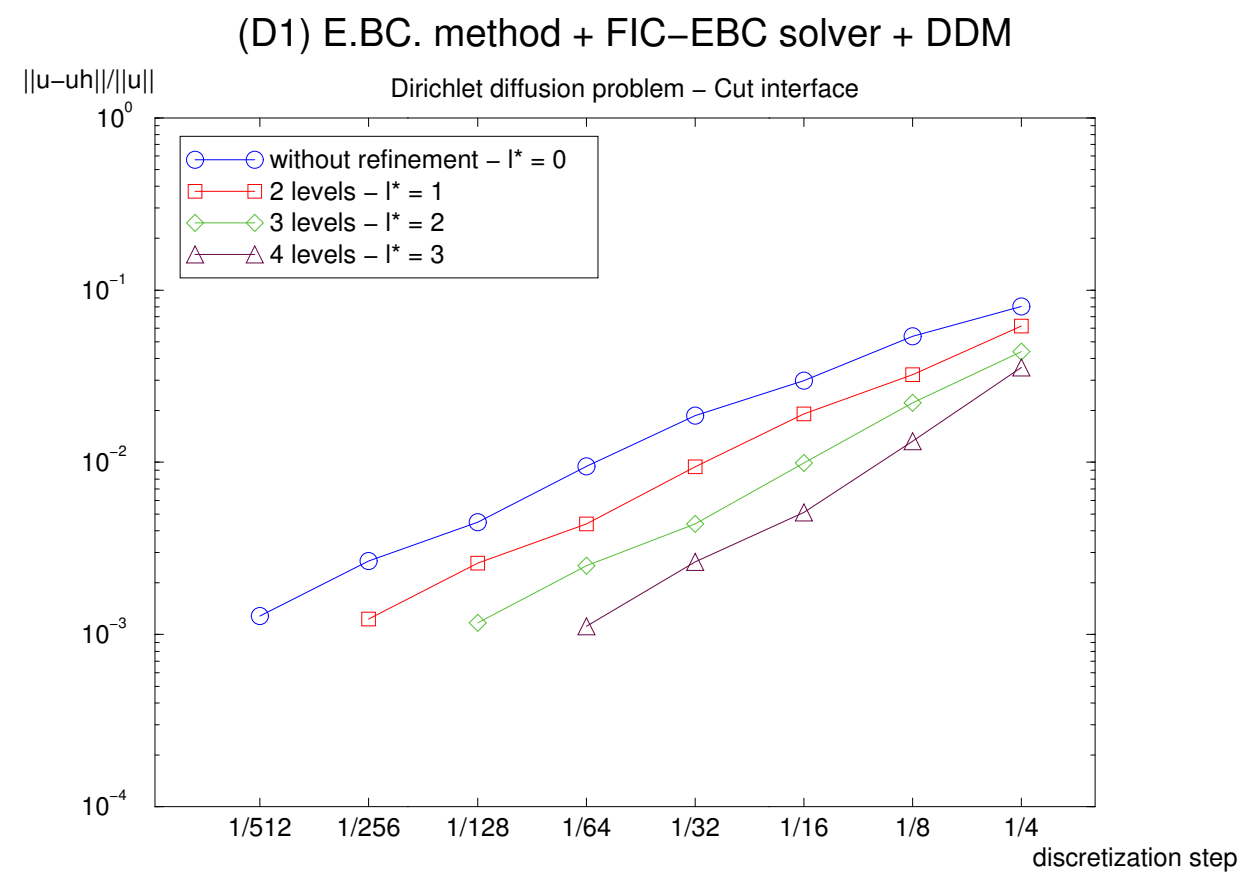

(b) Cut interface $\Sigma_{h}^{c u t}$.

Figure 13. Convergence for the $L^{2}$-norm of the discretization error with $h_{0}$ for the combination of the $(D 1)$ E.B.C. method with the FIC-EBC solver for the Dirichlet diffusion problem. 


\begin{tabular}{|c|c|c|c|c|c|c|c|c|}
\hline \multicolumn{9}{|c|}{ Exterior interface $\Sigma_{h}^{e x t}$} \\
\hline$l^{\star} h_{0}$ & $1 / 4$ & $1 / 8$ & $1 / 16$ & $1 / 32$ & $1 / 64$ & $1 / 128$ & $1 / 256$ & $1 / 512$ \\
\hline 0 & $1.9510^{-3}$ & $1.9510^{-3}$ & $3.9010^{-3}$ & $3.3210^{-2}$ & $2.3810^{-1}$ & $6.2610^{0}$ & $6.0210^{1}$ & $4.8710^{2}$ \\
\hline 1 & $1.7910^{-1}$ & $3.9010^{-1}$ & $1.0310^{0}$ & $3.0510^{0}$ & $1.1610^{1}$ & $8.8810^{1}$ & $4.7810^{2}$ & \\
\hline 2 & $9.4110^{-1}$ & $2.3110^{0}$ & $5.9910^{0}$ & $1.8010^{1}$ & $5.9810^{1}$ & $2.4410^{2}$ & & \\
\hline 3 & $3.3510^{0}$ & $8.4410^{0}$ & $2.4110^{1}$ & $7.3510^{1}$ & $2.2410^{2}$ & & & \\
\hline \multicolumn{9}{|c|}{ Cut interface $\Sigma_{h}^{\text {cut }}$} \\
\hline$l^{\star} h_{0}$ & $1 / 4$ & $1 / 8$ & $1 / 16$ & $1 / 32$ & $1 / 64$ & $1 / 128$ & $1 / 256$ & $1 / 512$ \\
\hline 0 & $1.9510^{-3}$ & $1.9510^{-3}$ & $5.8610^{-3}$ & $3.1210^{-2}$ & $2.3410^{-1}$ & $5.7610^{0}$ & $5.5310^{1}$ & $4.9710^{2}$ \\
\hline 1 & $1.4210^{-1}$ & $3.5510^{-1}$ & $8.7310^{-1}$ & $2.7610^{0}$ & $1.0110^{1}$ & $7.4610^{1}$ & $4.1710^{2}$ & \\
\hline 2 & $7.9010^{-1}$ & $1.9910^{0}$ & $5.2010^{0}$ & $1.5210^{1}$ & $4.6210^{1}$ & $1.9610^{2}$ & & \\
\hline 3 & $2.8610^{0}$ & $7.2810^{0}$ & $1.9610^{1}$ & $5.8910^{1}$ & $1.8410^{2}$ & & & \\
\hline
\end{tabular}

Table 2

CPU time (in seconds) with respect to $h_{0}$ and $l^{\star}$ for the (D1) E.B.C. method combined with the FIC-EBC solver for the Dirichlet diffusion problem.

As we explained in Section 4.3.2.3, the DDM iterations are necessary to recover the good solution with a cut interface approximation $\Sigma_{h}^{c u t}$ of $\Sigma$. On the other hand, for the exterior interface $\Sigma_{h}^{e x t}$, at each level $l+1$, all the control volumes $K_{l+1} \in G_{l+1}^{i n t}, K_{l+1} \subset \tilde{\Omega}_{h_{l+1}}$ have their parent cell $K_{l} \subset \tilde{\Omega}_{h_{l}}$. Fig. 15(a) shows that, even without DDM iterations, the convergence of the E.B.C. method combined with the FIC-EBC solver remains in $\mathcal{O}\left(h_{l^{\star}}\right)$ for $\Sigma_{h}^{e x t}$. In this case the CPU time is reduced (see Table 3 and Fig. 15(b)). Indeed, to obtain the error of $h_{0}=\frac{1}{512}$ and $l^{\star}=0$ using the FIC-EBC solver with $h_{0}=\frac{1}{128}$ and $l^{\star}=2$, the CPU time is divided by a factor 9 .

Since the method with the exterior interface $\Sigma_{h}^{e x t}$ does not require DDM iterations, the ratio of the precision of the solution over the CPU time is better for this method than for the method with a cut interface $\Sigma_{h}^{c u t}$. To avoid the DDM smoothing iterations for the cut interface approach, some patches (of the same level of refinement) have to be gathered. 
(D1) E.B.C. method + FIC-EBC solver + DDM

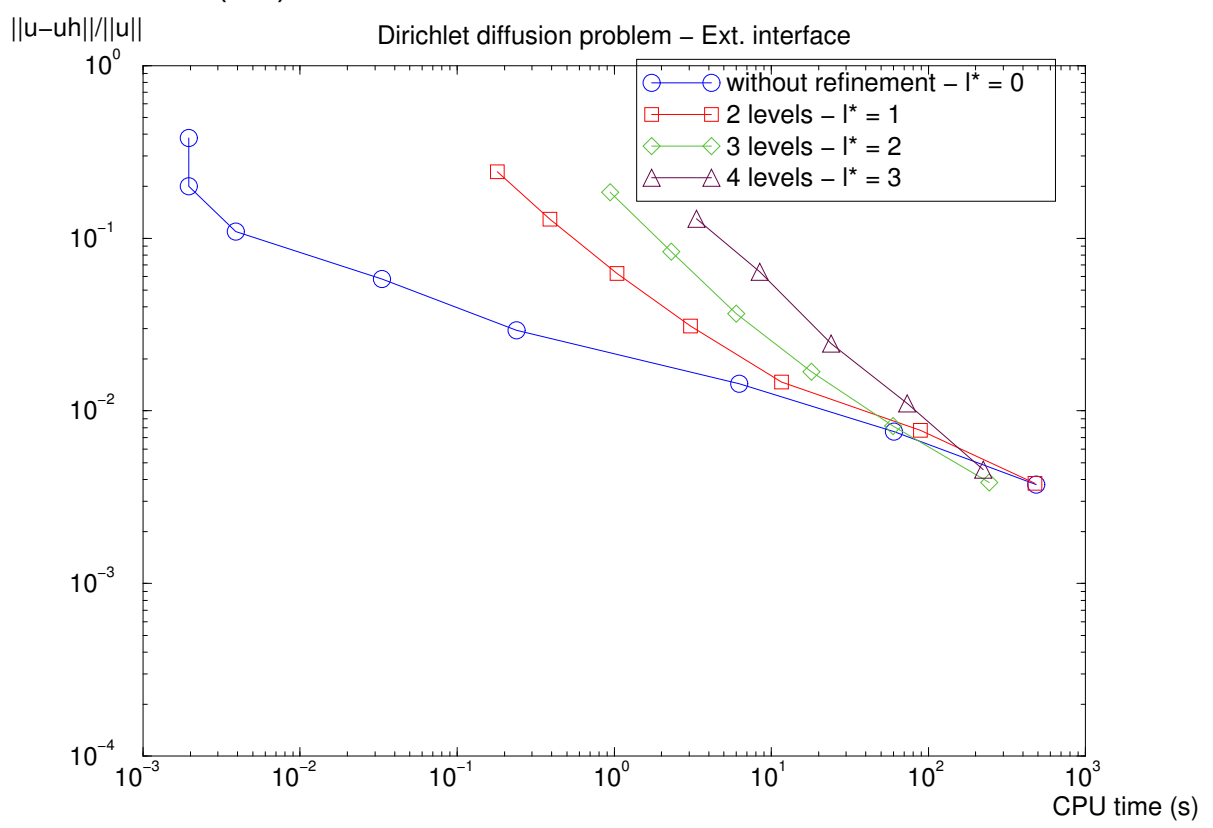

(a) Exterior interface $\Sigma_{h}^{e x t}$.

(D1) E.B.C. method + FIC-EBC solver + DDM

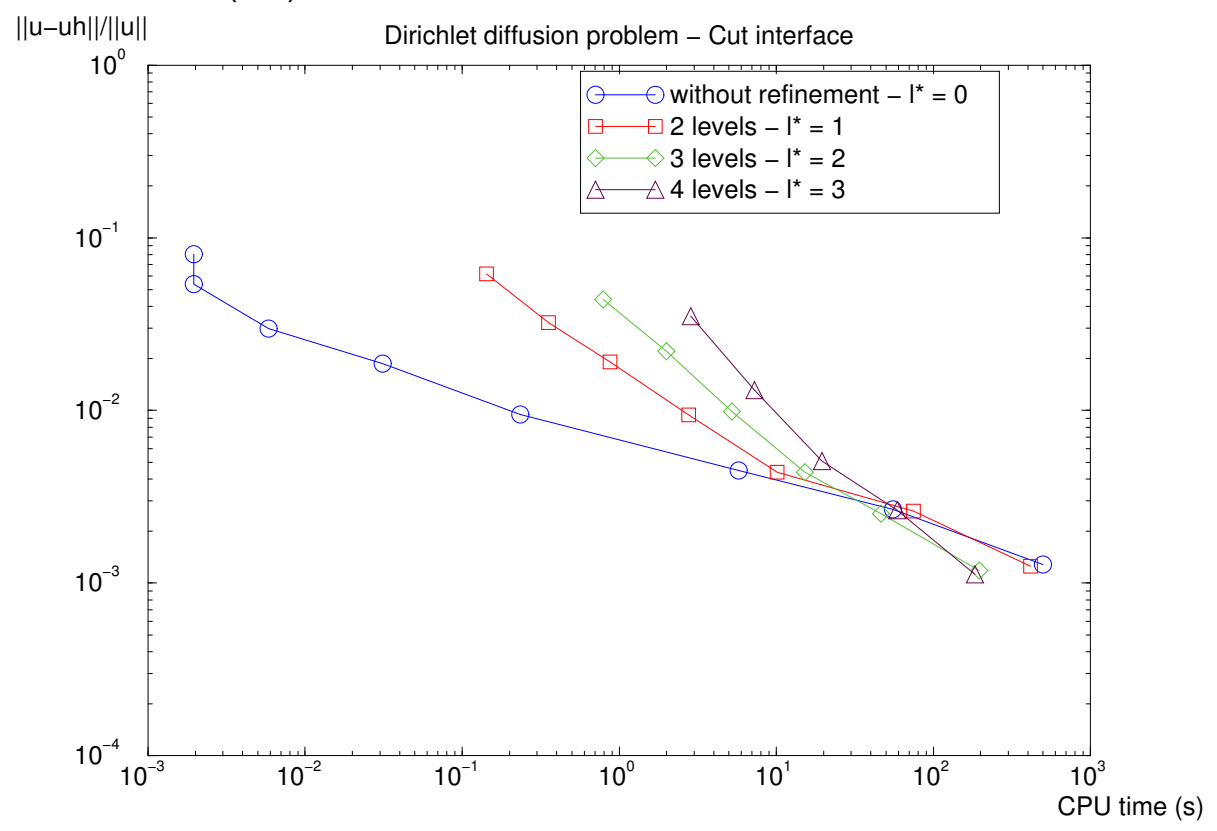

(b) Cut interface $\Sigma_{h}^{c u t}$.

Figure 14. Convergence for the $L^{2}$-norm of the discretization error with the CPU time for the combination of the (D1) E.B.C. method with the FIC-EBC solver for the Dirichlet diffusion problem. 
(D1) E.B.C method + FIC-EBC solver

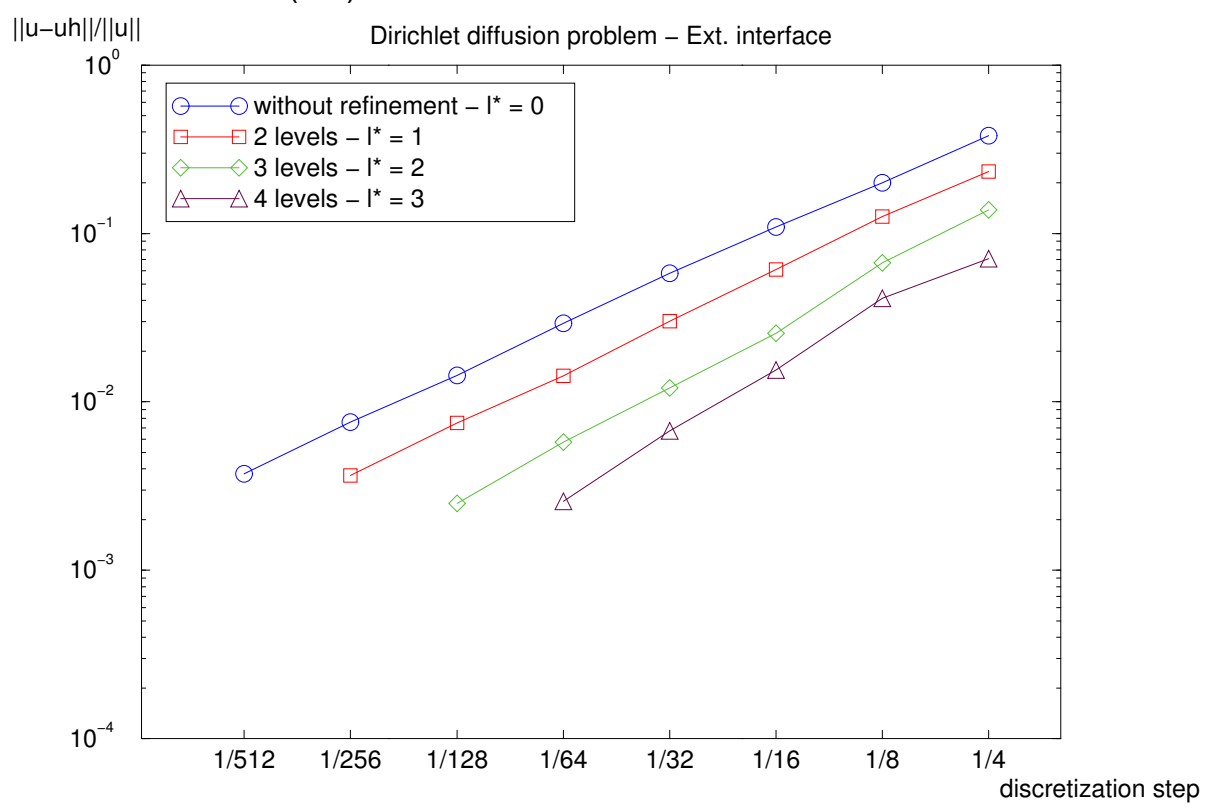

(a) Error versus $h_{0}$.

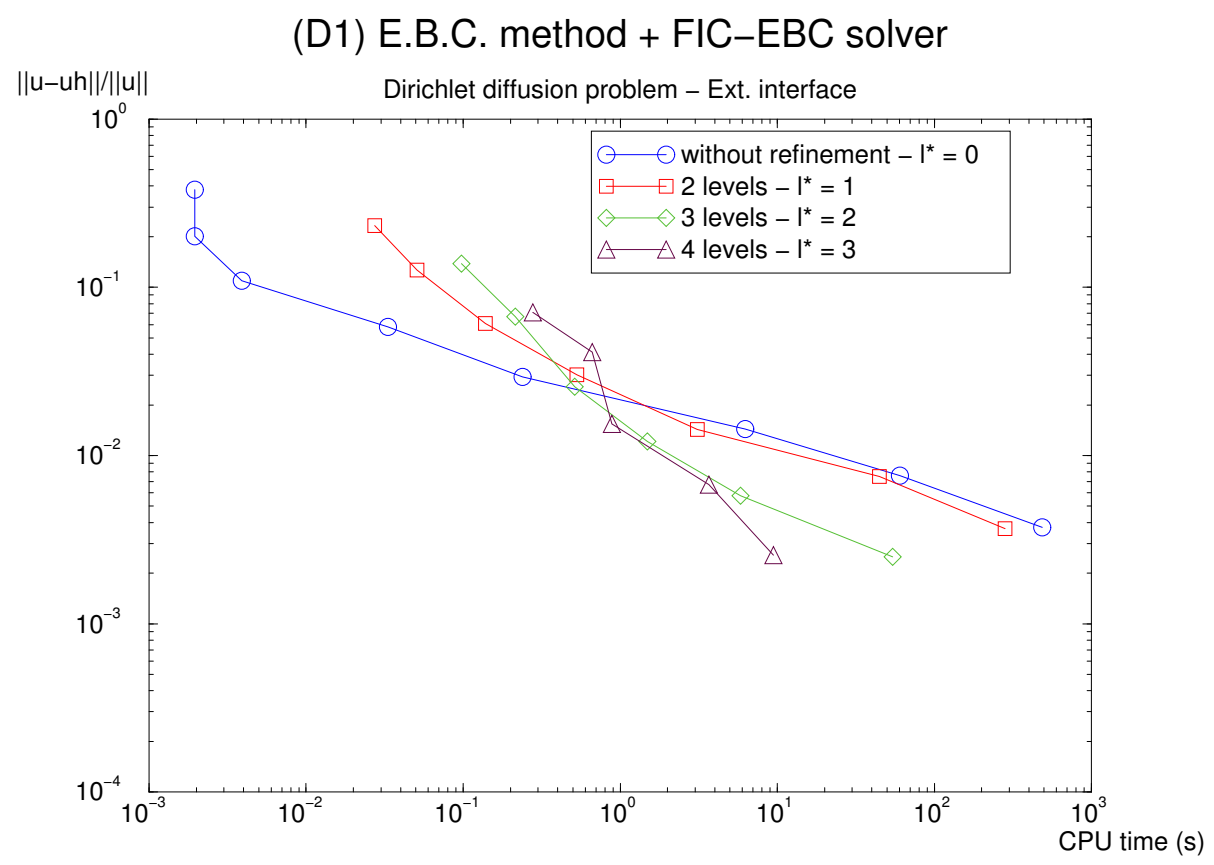

(b) Error versus CPU time.

Figure 15. Convergence for the $L^{2}$-norm of the discretization error for the combination of the $(D 1)$ E.B.C. method with the FIC-EBC solver without DDM iterations for the Dirichlet diffusion problem with $\Sigma_{h}^{e x t}$. 


\begin{tabular}{|c|l|l|l|l|l|l|l|l|}
\hline \multicolumn{8}{|c|}{ Exterior interface $\Sigma_{h}^{e x t}($ FIC-EBC solver without DDM) } \\
\hline$l^{\star} h_{0}$ & $1 / 4$ & $1 / 8$ & $1 / 16$ & $1 / 32$ & $1 / 64$ & $1 / 128$ & $1 / 256$ & $1 / 512$ \\
\hline 0 & $1.9510^{-3}$ & $1.9510^{-3}$ & $3.9010^{-3}$ & $3.3210^{-2}$ & $2.3810^{-1}$ & $6.2610^{0}$ & $6.0210^{1}$ & $4.8710^{2}$ \\
\hline 1 & $2.7310^{-2}$ & $5.0810^{-2}$ & $1.3910^{-1}$ & $5.2910^{-1}$ & $3.0910^{0}$ & $4.4610^{1}$ & $2.8310^{2}$ & \\
\hline 2 & $9.7710^{-2}$ & $2.1510^{-1}$ & $5.1210^{-1}$ & $1.4910^{0}$ & $5.8510^{0}$ & $5.4310^{1}$ & & \\
\hline 3 & $2.7710^{-1}$ & $6.6210^{-1}$ & $8.8710^{-1}$ & $3.6810^{0}$ & $9.4610^{0}$ & & & \\
\hline
\end{tabular}

Table 3

CPU time (in seconds) with respect to $h_{0}$ and $l^{\star}$ for the (D1) E.B.C. method combined with the FIC-EBC solver without DDM iterations for the Dirichlet diffusion problem with $\Sigma_{h}^{e x t}$.

In order to validate the $(D 1)$ E.B.C. method for any Dirichlet boundary conditions, we now study a nonhomogeneous Dirichlet problem

$$
(\tilde{\mathcal{P}})\left\{\begin{aligned}
-\triangle \tilde{u} & =-\left(2+4 x^{2}\right) \exp \left(x^{2}\right) \quad & & \text { in } \tilde{\Omega}, \\
\frac{\partial \tilde{u}}{\partial n} & =0 & & \text { on } \tilde{\Gamma}, \\
\tilde{u}=u_{D} & =3+\exp \left(\cos ^{2} \theta\right) & & \text { on } \Sigma\left(\text { where } \theta=\arctan \left(\frac{y}{x}\right)\right),
\end{aligned}\right.
$$

which has the analytic solution

$$
\tilde{u}=3+\exp \left(x^{2}\right) \quad \text { in } \tilde{\Omega} .
$$

The fictitious problem over $\Omega$ is solved using the (D1) E.B.C. method. As Figure 16(a) shows, the first-order accuracy is reached for the $L^{2}$-norm for both approximate immersed interfaces. Since the method involving the cut interface is more accurate, the FIC-EBC solver is combined with the (D1) E.B.C. method for the cut interface. The results reported in Figure 16(b) exhibit a first-order convergence of the method with respect to the local finest grid's discretization step $h_{l^{\star}}$. This confirms that the (D1) E.B.C. method enables to take account for any Dirichlet embedded boundary condition and that the combination with the multilevel algorithm leads to an $\mathcal{O}\left(h_{l^{\star}}\right)$ asymptotic convergence for the $L^{2}$-norm. 


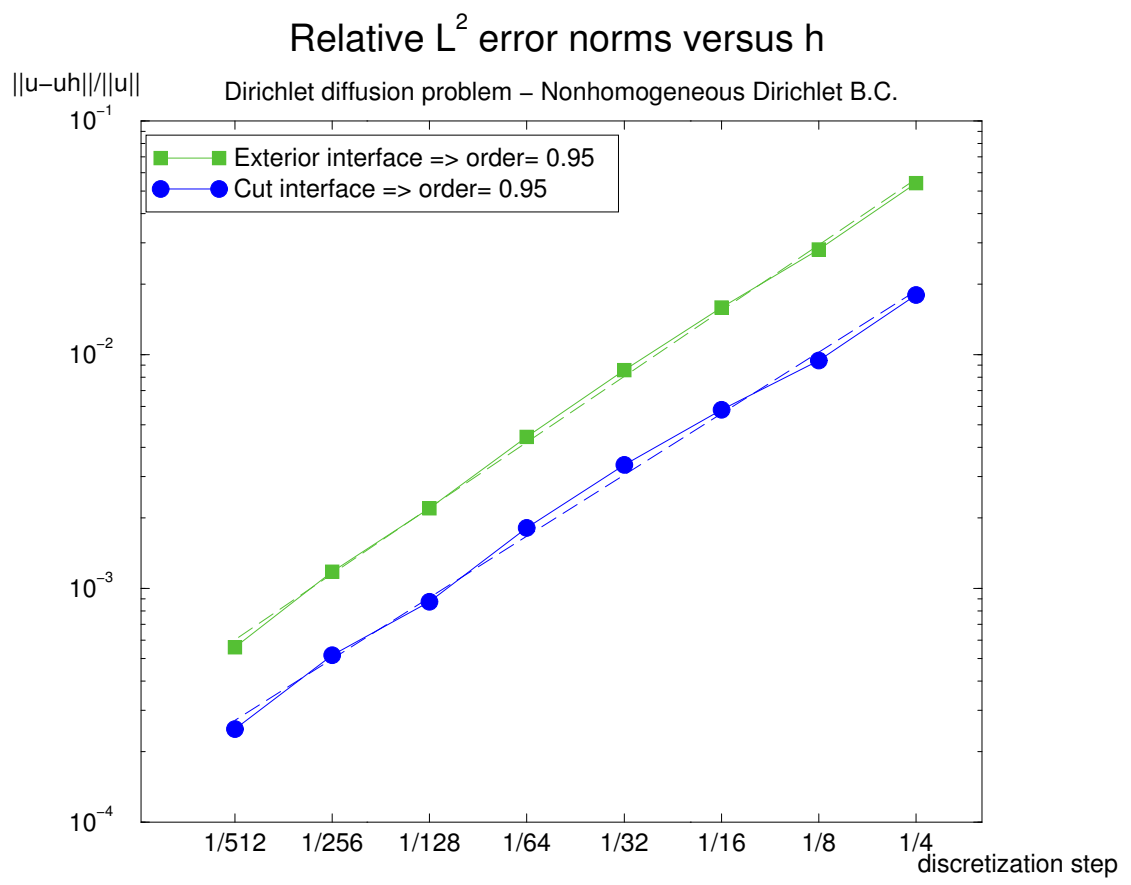

(a) (D1) E.B.C. method with $\Sigma_{h}^{e x t}$ and $\Sigma_{h}^{c u t}$.

(D1) E.B.C. method + FIC-EBC solver + DDM

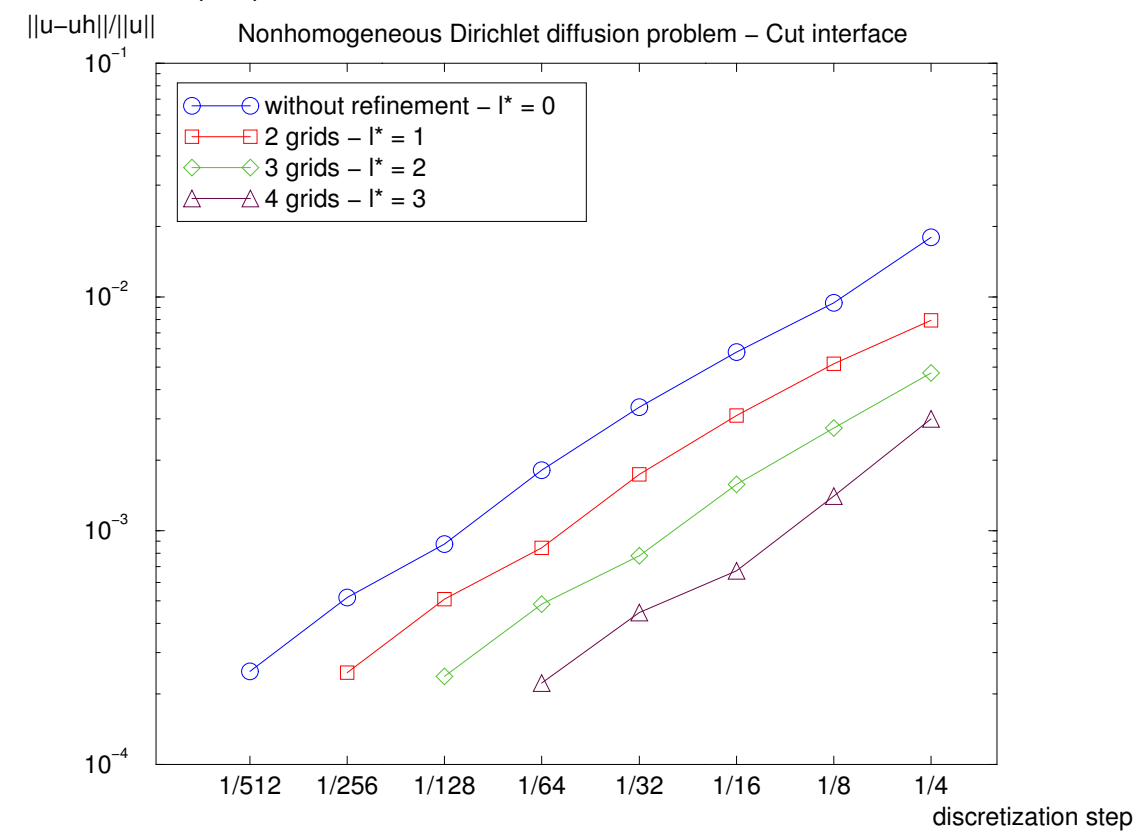

(b) Combination with the FIC-EBC solver for $\Sigma_{h}^{c u t}$.

Figure 16. Convergence for the $L^{2}$-norm of the discretization error with $h_{0}$ for the nonhomogeneous Dirichlet diffusion problem with $\eta=10^{-12}$. 


\subsubsection{Robin problem}

We now consider the Robin problem

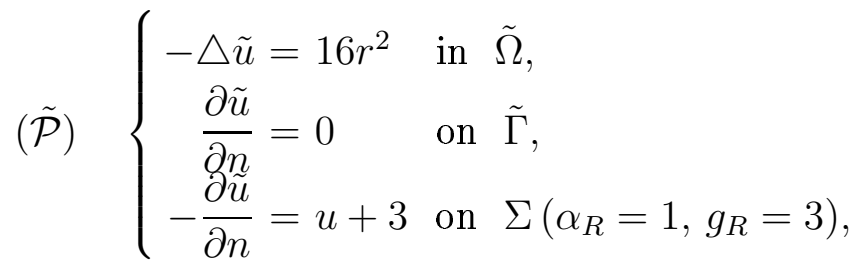

which has the analytic solution

$$
\tilde{u}=2-r^{4} \quad \text { in } \tilde{\Omega} \text { with } r=\sqrt{x^{2}+y^{2}} .
$$

The fictitious domain problem is solved in $\Omega$ with the E.B.C. method $(R)$ without exterior control, as described in the Table 1. We investigate in Fig. 17(a) the effect of the global correction for the approximate interface proposed in Section 3.3 where $\epsilon_{h}$ is computed by Eq. (28) in each finite volume crossed by $\Sigma$. An asymptotic stagnation of the error can be observed and the first-order precision is lost. For a local correction $\epsilon_{K}$, if a local formula (see Eq. (23)) is applied without taking into account the control volumes $K \subset \omega_{h, \Sigma}$ with $\hat{\mathcal{E}}_{K}=\emptyset$, there is no error convergence (see Fig. 17(b)).

When the accurate local correction $\epsilon_{K}$ (see Eq. (26)) taking account of all the local measures of $\Sigma_{l, K}$ for $K \subset \omega_{h, \Sigma}$ is computed, the first-order accuracy is then yielded for the $L^{2}$-norm error, see Fig. 18. Here again, the approximate interface $\Sigma_{h}^{c u t}$ gives a better precision than the approximate interface $\Sigma_{h}^{e x t}$.

The $(R)$ E.B.C. with the accurate local correction is then combined with the FIC-EBC solver. The results reported in Fig. 19 show a $L^{2}$-norm asymptotic accuracy of the method in $\mathcal{O}\left(h_{l^{\star}}\right), h_{l^{\star}}$ being the discretization step of the local finest grid.

As we can observe in Table 4 and Fig. 20, the combination of the E.B.C. method with the FIC-EBC solver improves the ratio of the obtained precision over the CPU time from a relative precision of $10^{-1}$. This improvement is better than for the Dirichlet case: the CPU ratio is around 15 between the error obtained with $h_{0}=\frac{1}{512}$ and $l^{\star}=0$ and the same error obtained with $h_{0}=\frac{1}{128}$ and $l^{\star}=2$.

As in the Dirichlet case, Fig. 21(a) enables us to conclude that DDM iterations are not required for the exterior interface $\Sigma_{h}^{e x t}$. The relative CPU time is more improved (see Table 5 and Fig. 21(b)). The CPU time ratio reaches 25 between the same error obtained either with $h_{0}=\frac{1}{512}$ and $l^{\star}=0$ or with $h_{0}=\frac{1}{128}$ and $l^{\star}=2$. However, even looking at the results in term of CPU 


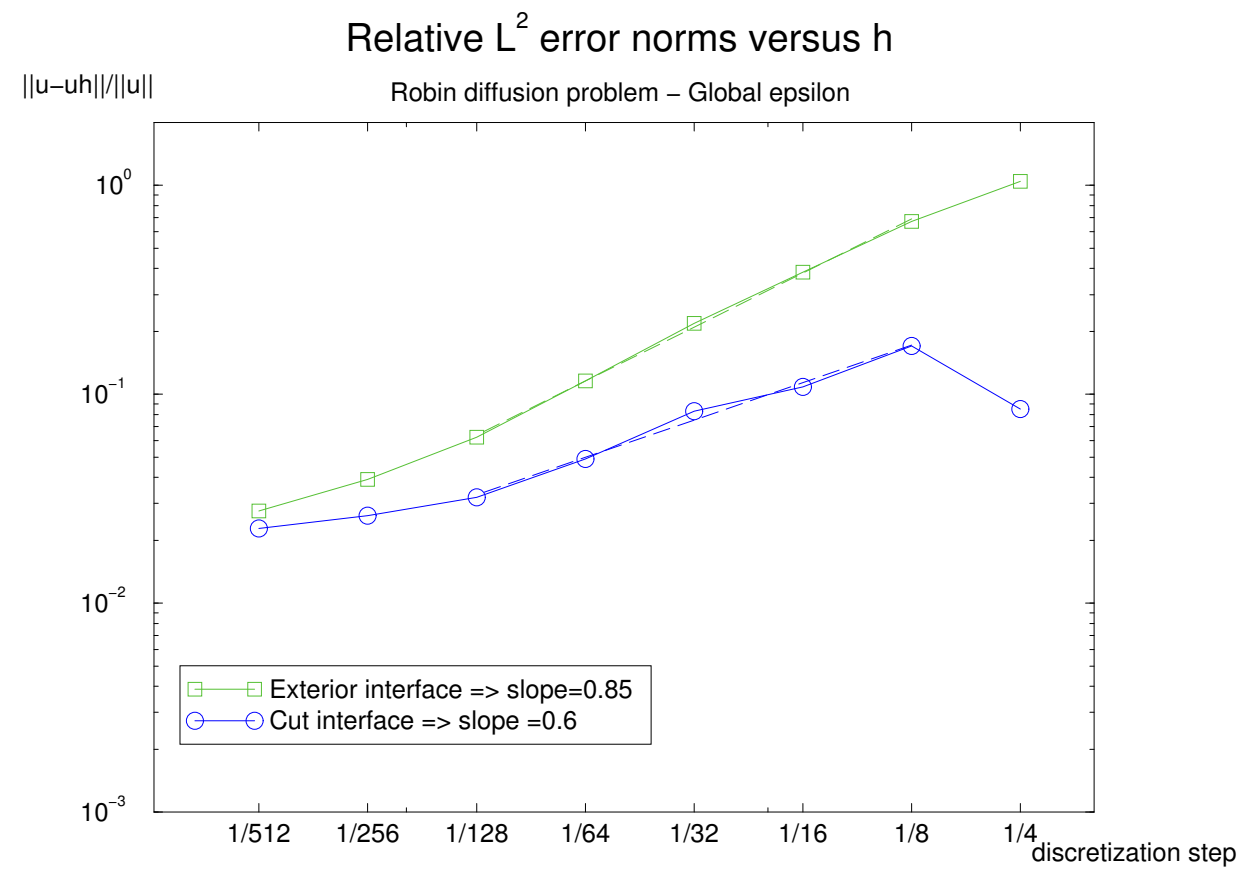

(a) Global correction with $\epsilon_{h}$.

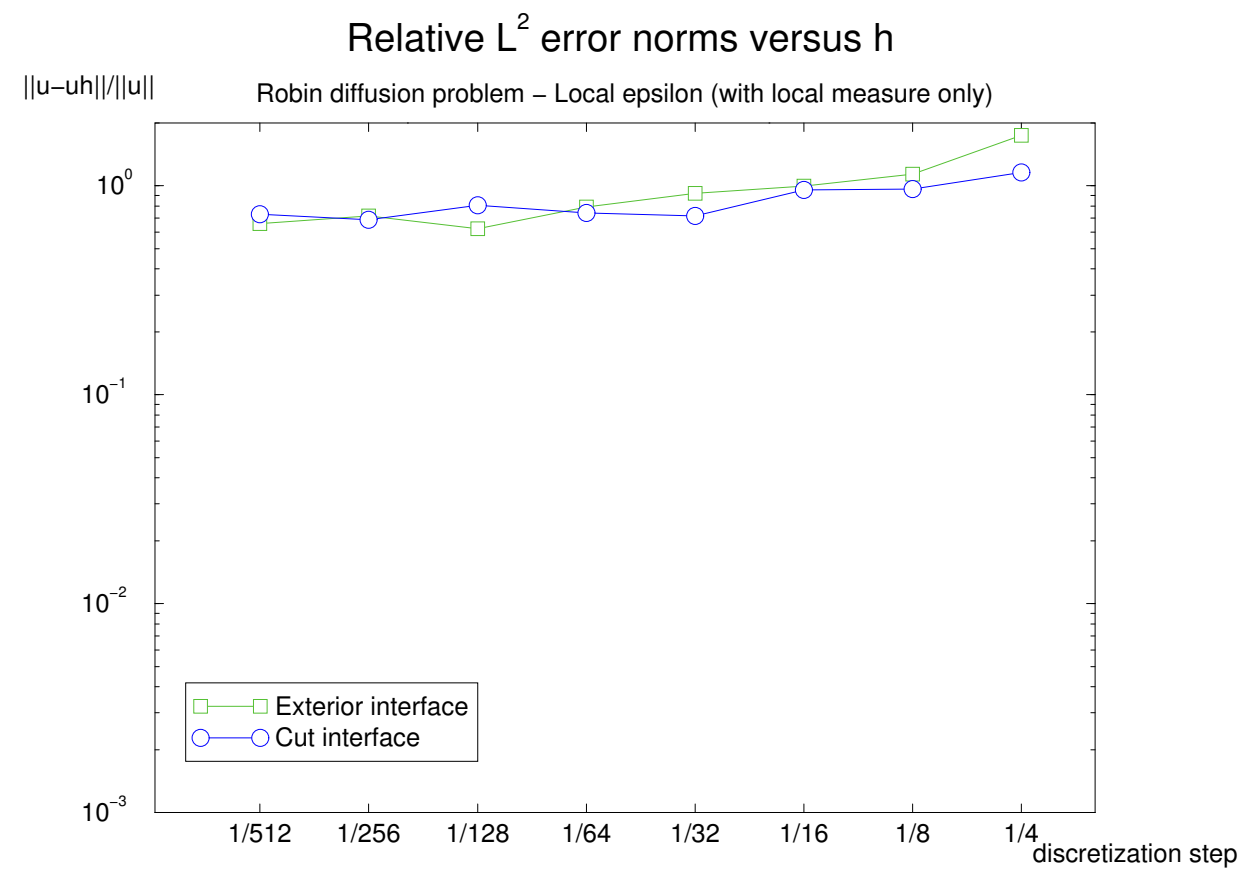

(b) Local correction $\epsilon_{K}$ without correction of $\Sigma_{l, K}$.

Figure 17. Convergence for the $L^{2}$-norm of the discretization error with $h$ of the $(R)$ E.B.C. method for the Robin problem with $\Sigma_{h}^{e x t}$ or $\Sigma_{h}^{c u t}$ : global correction and local correction without extension of the local surface $\Sigma_{l, K}$. 


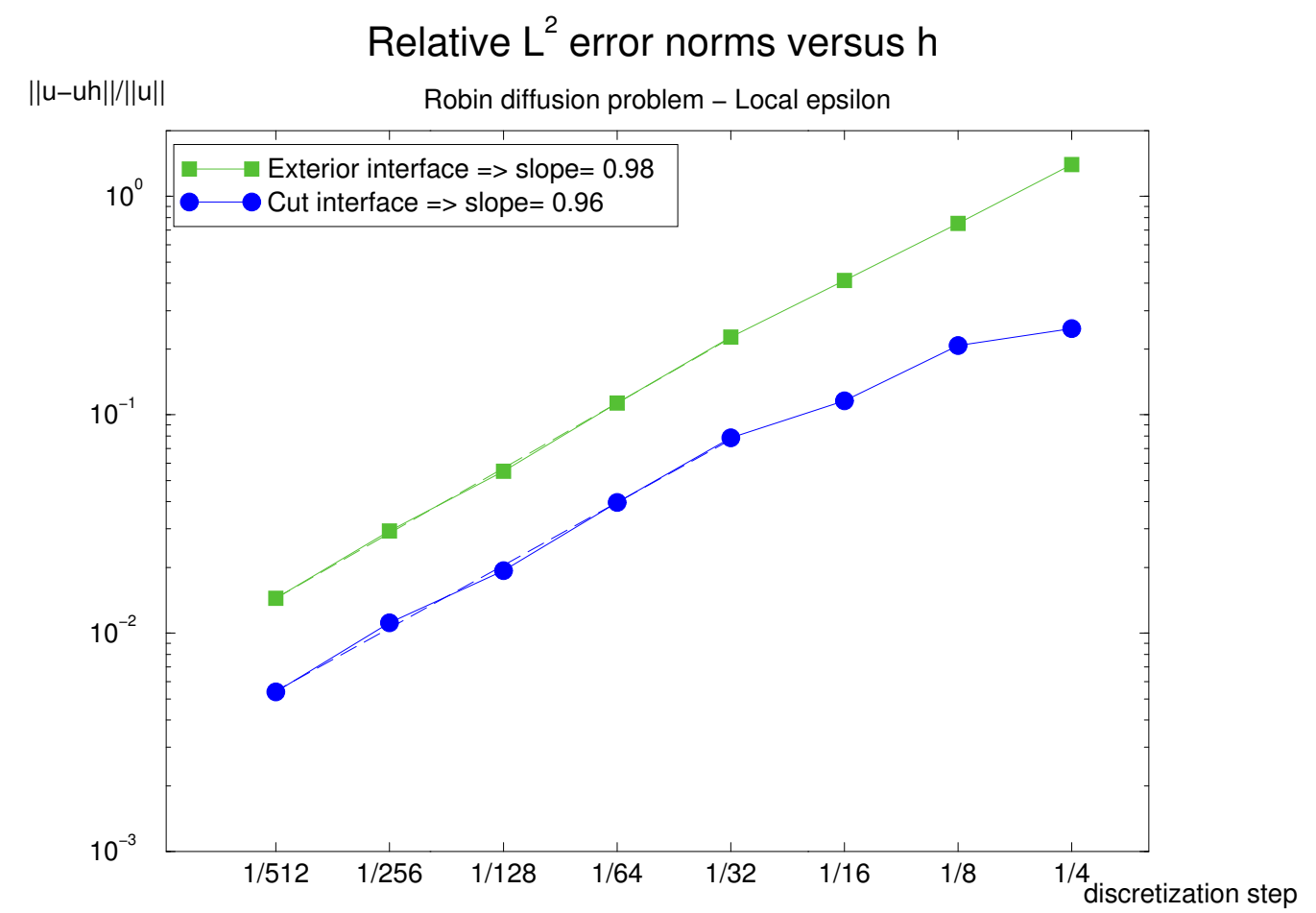

Figure 18. Convergence for the $L^{2}$-norm of the discretization error with $h$ of the $(R)$ E.B.C. method for the Robin problem with $\Sigma_{h}^{e x t}$ or $\Sigma_{h}^{c u t}$ : local correction with extension of the local surface $\Sigma_{l, K}$.

\begin{tabular}{|c|l|l|l|l|l|l|l|l|}
\hline \multicolumn{8}{|c|}{ Exterior interface $\Sigma_{h}^{e x t}$} \\
\hline$l^{\star} h_{0}$ & $1 / 4$ & $1 / 8$ & $1 / 16$ & $1 / 32$ & $1 / 64$ & $1 / 128$ & $1 / 256$ & $1 / 512$ \\
\hline 0 & $3.9010^{-3}$ & $3.9010^{-3}$ & $5.8610^{-3}$ & $3.7110^{-2}$ & $2.8110^{-1}$ & $7.4310^{0}$ & $8.0410^{1}$ & $5.8910^{2}$ \\
\hline 1 & $4.2910^{-2}$ & $9.7710^{-2}$ & $2.1310^{-1}$ & $6.7610^{-1}$ & $2.3310^{0}$ & $2.1710^{1}$ & $1.7710^{2}$ & \\
\hline 2 & $2.2210^{-1}$ & $4.9210^{-1}$ & $9.0410^{-1}$ & $3.4410^{0}$ & $9.8410^{0}$ & $5.1710^{1}$ & & \\
\hline 3 & $7.6610^{-1}$ & $1.5510^{0}$ & $3.0510^{0}$ & $1.1910^{1}$ & $4.0110^{1}$ & & & \\
\hline \hline \multicolumn{7}{|c|}{ Cut interface $\Sigma_{h}^{c u t}$} & & \\
\hline$l^{\star}$ & $1 / 4$ & $1 / 8$ & $1 / 16$ & $1 / 32$ & $1 / 64$ & $1 / 128$ & $1 / 256$ & $1 / 512$ \\
\hline 0 & $1.9510^{-3}$ & $1.9510^{-3}$ & $5.8610^{-3}$ & $3.7110^{-2}$ & $2.8910^{-1}$ & $7.2210^{0}$ & $7.7210^{1}$ & $6.1810^{2}$ \\
\hline 1 & $3.9010^{-2}$ & $9.7710^{-2}$ & $2.0710^{-1}$ & $6.4410^{-1}$ & $2.1710^{0}$ & $2.2010^{1}$ & $1.8310^{2}$ & \\
\hline 2 & $2.1310^{-1}$ & $4.0410^{-1}$ & $8.7710^{-1}$ & $2.8410^{0}$ & $9.6710^{0}$ & $4.0810^{1}$ & & \\
\hline 3 & $6.5610^{-1}$ & $1.5010^{0}$ & $3.7510^{0}$ & $9.8610^{0}$ & $4.0110^{1}$ & & & \\
\hline
\end{tabular}

Table 4

CPU time (in seconds) with respect to $h_{0}$ and $l^{\star}$ for the $(R)$ E.B.C. method combined with the FIC-EBC solver for the Robin diffusion problem. 
(R) E.B.C. method + FIC-EBC solver + DDM

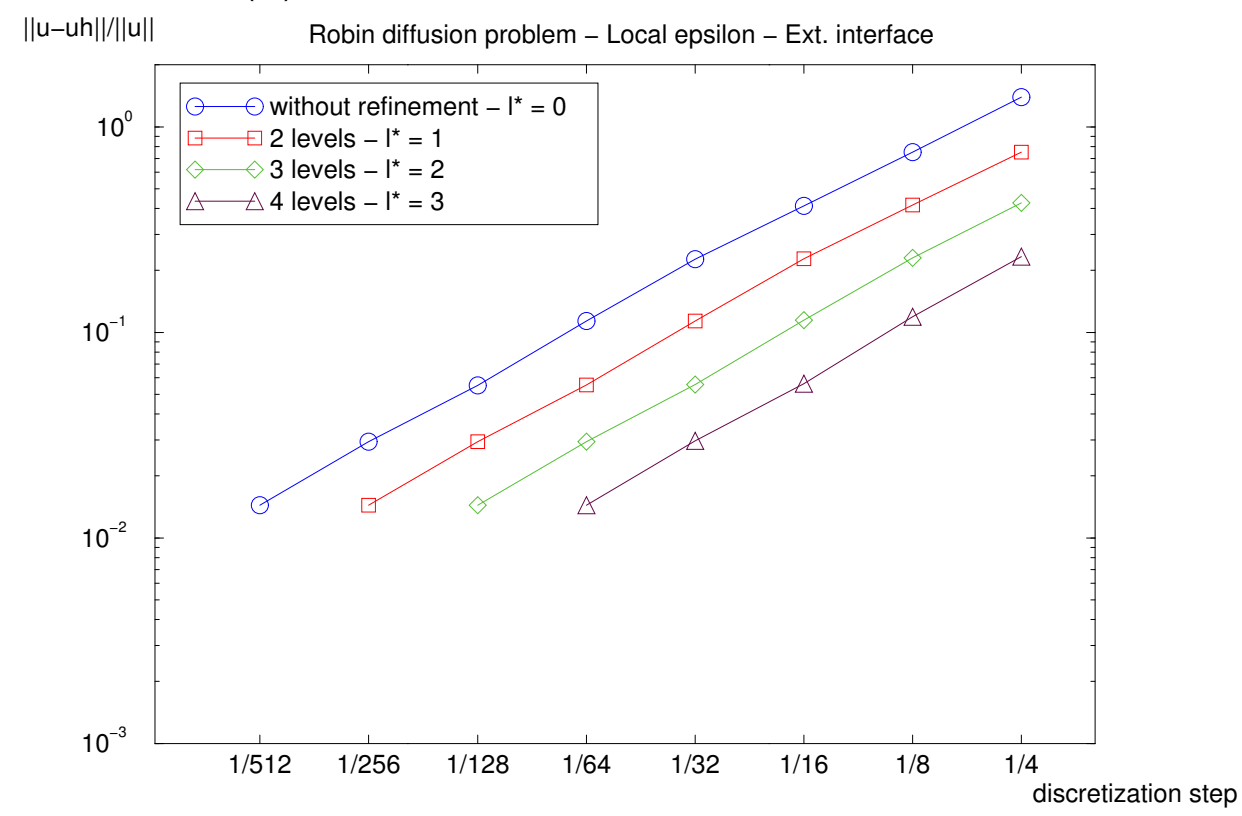

(a) Exterior interface $\Sigma_{h}^{e x t}$.

(R) E.B.C. method + FIC-EBC solver + DDM

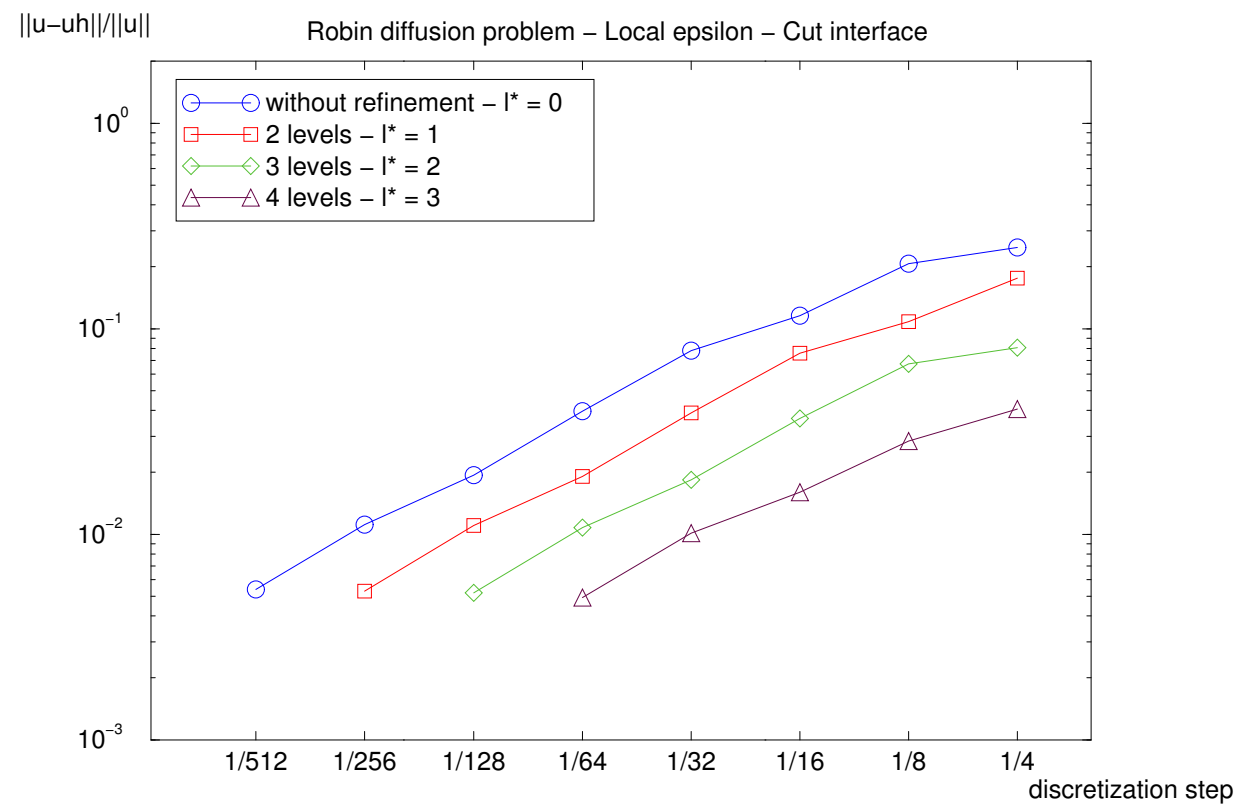

(b) Cut interface $\Sigma_{h}^{c u t}$.

Figure 19. Convergence for the $L^{2}$-norm of the discretization error with $h_{0}$ for the combination of the $(R)$ E.B.C. method with the FIC-EBC solver for the Robin diffusion problem with a local correction $\epsilon_{K}$. 
(R) E.B.C. method + FIC-EBC solver + DDM

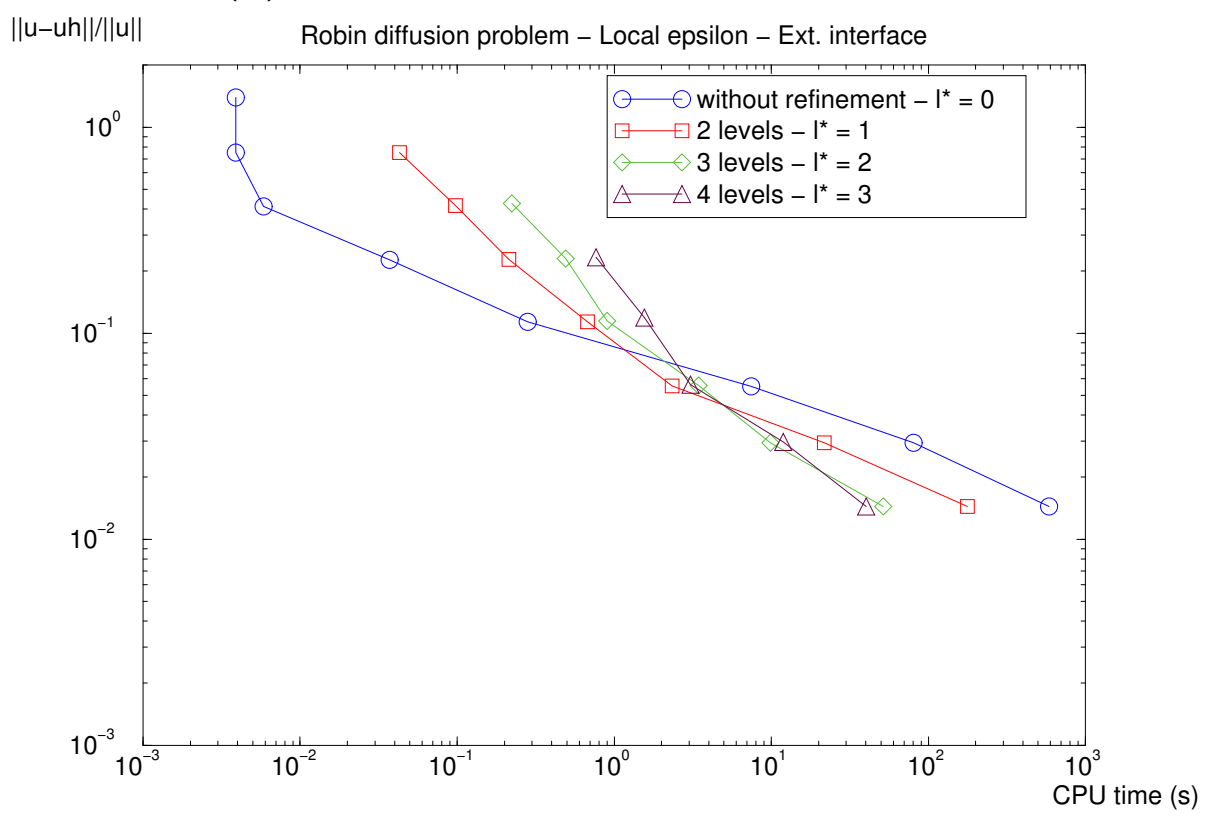

(a) Exterior interface $\Sigma_{h}^{e x t}$.

(R) E.B.C. method + FIC-EBC solver + DDM

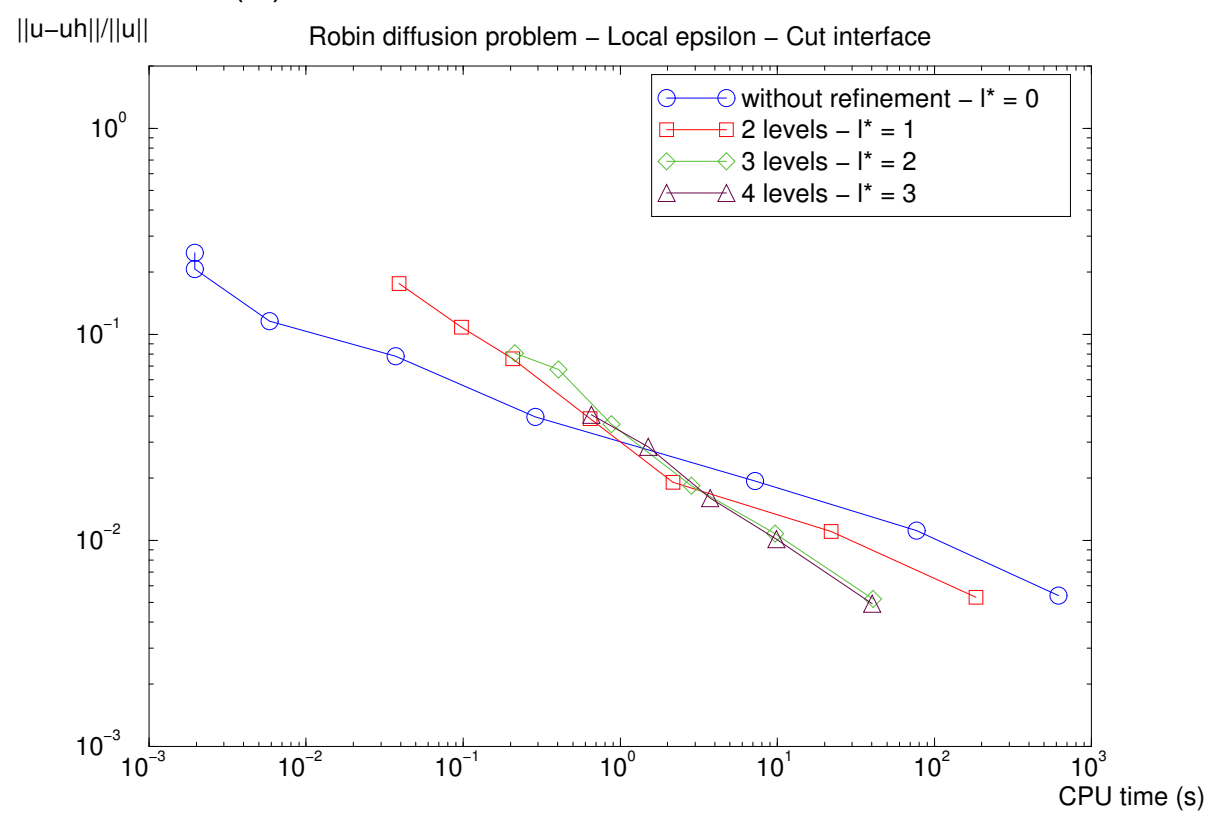

(b) Cut interface $\Sigma_{h}^{c u t}$.

Figure 20. Convergence for the $L^{2}$-norm of the discretization error with CPU time for the combination of the $(R)$ E.B.C. method with the FIC-EBC solver for the Robin diffusion problem. 
time, the method with the cut interface $\Sigma_{h}^{c u t}$ and DDM iterations is the most accurate in the Robin case.

\begin{tabular}{|c|l|l|l|l|l|l|l|l|}
\hline \multicolumn{7}{|c|}{ Exterior interface $\Sigma_{h}^{e x t}($ FIC-EBC solver without DDM) } \\
\hline$l^{\star} h_{0}$ & $1 / 4$ & $1 / 8$ & $1 / 16$ & $1 / 32$ & $1 / 64$ & $1 / 128$ & $1 / 256$ & $1 / 512$ \\
\hline 0 & $3.9010^{-3}$ & $3.9010^{-3}$ & $5.8610^{-3}$ & $3.7110^{-2}$ & $2.8110^{-1}$ & $7.4310^{0}$ & $8.0410^{1}$ & $5.8910^{2}$ \\
\hline 1 & $7.8110^{-3}$ & $2.3410^{-2}$ & $5.2710^{-2}$ & $2.3210^{-1}$ & $1.4010^{0}$ & $2.1110^{1}$ & $1.7310^{2}$ & \\
\hline 2 & $3.7110^{-2}$ & $7.4210^{-2}$ & $1.7210^{-1}$ & $5.2510^{-1}$ & $1.9110^{0}$ & $2.4010^{1}$ & & \\
\hline 3 & $9.5710^{-2}$ & $1.6210^{-1}$ & $5.2110^{-1}$ & $1.1710^{0}$ & $3.6010^{0}$ & & & \\
\hline
\end{tabular}

Table 5

CPU time (in seconds) with respect to $h_{0}$ and $l^{\star}$ for the $(R)$ E.B.C. method combined with the FIC-EBC solver without DDM iterations for the Robin diffusion problem with $\Sigma_{h}^{e x t}$.

REMARK. The convergence results for the $L^{\infty}$-norm are similar to those presented for the $L^{2}$-norm (see the curves plotted for convection-diffusion problems in the next section):

$$
\left\|\tilde{u}-u_{h}\right\|_{L^{\infty}\left(\tilde{\Omega}_{h}\right)}=\mathcal{O}\left(h_{l^{\star}}\right)
$$

and

$$
\left\|\tilde{u}-u_{h}\right\|_{L^{\infty}\left(\tilde{\Omega}_{h}\right)}=\mathcal{O}\left(h_{0}\right) \text { when the residual stagnation error is reached. }
$$

\subsubsection{Convection-diffusion problems}

\subsubsection{Dirichlet problem}

The convection-diffusion Dirichlet problem is considered as follows

$$
\left\{\begin{aligned}
-\Delta \tilde{u}+\operatorname{div}(\tilde{\mathbf{v}} \tilde{u}) & =4 \text { in } \tilde{\Omega}, \\
\frac{\partial \tilde{u}}{\partial n}=0 & \text { on } \tilde{\Gamma} \\
\tilde{u}=u_{D}=0 & \text { on } \Sigma,
\end{aligned}\right.
$$

with $\tilde{\mathbf{v}}=\frac{r}{2} \mathbf{e}_{\mathbf{r}}$ where $r=\sqrt{x^{2}+y^{2}}$ and $\mathbf{e}_{\mathbf{r}}$ is the radial unit vector.

The analytic solution of this problem is :

$$
\tilde{u}=4\left(1-\exp \left(\frac{r^{2}-1}{4}\right)\right) \quad \text { in } \tilde{\Omega} .
$$

The associated fictitious problem $(\mathcal{P})$ is solved using either the $(D 1)$ or $(D 2)$ E.B.C method (see Table 1). The following results have been performed with 
(R) E.B.C. method + FIC-EBC solver

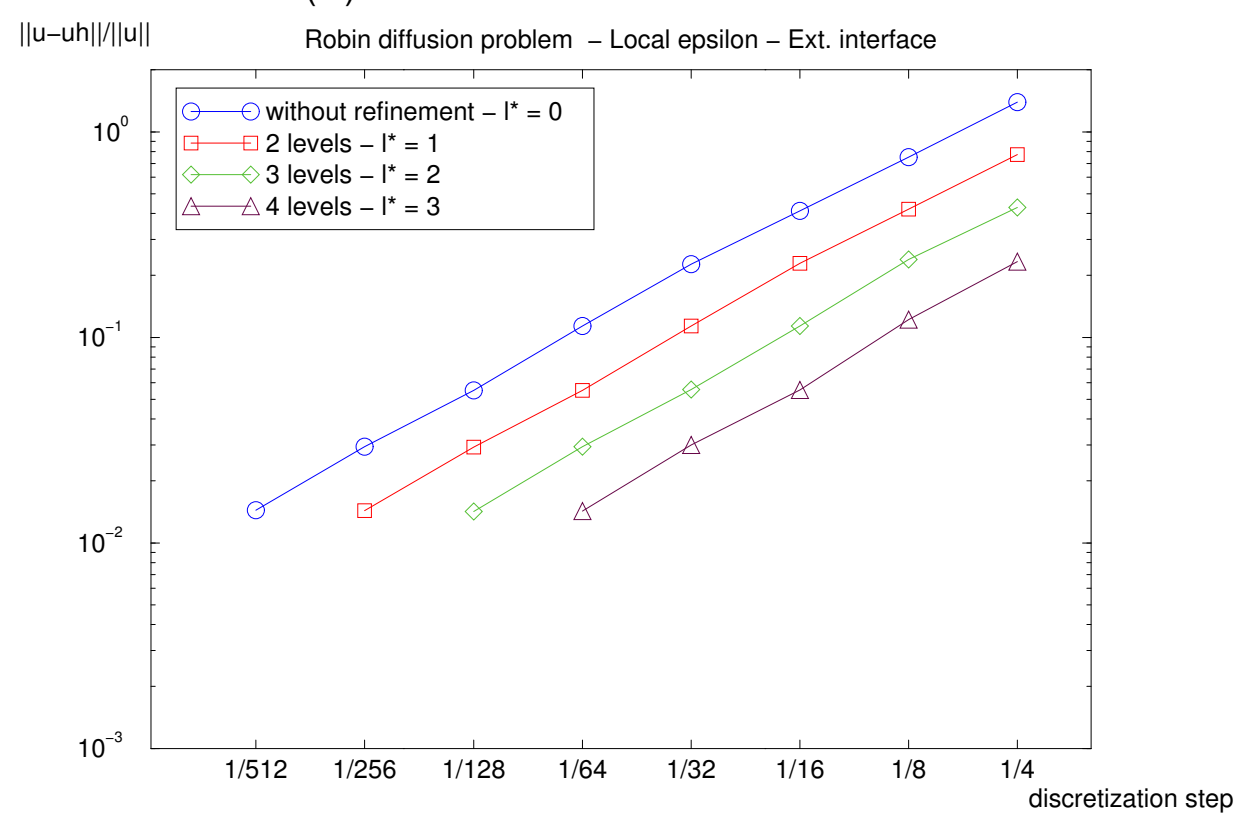

(a) Error versus $h_{0}$.

(R) E.B.C. method + FIC-EBC solver

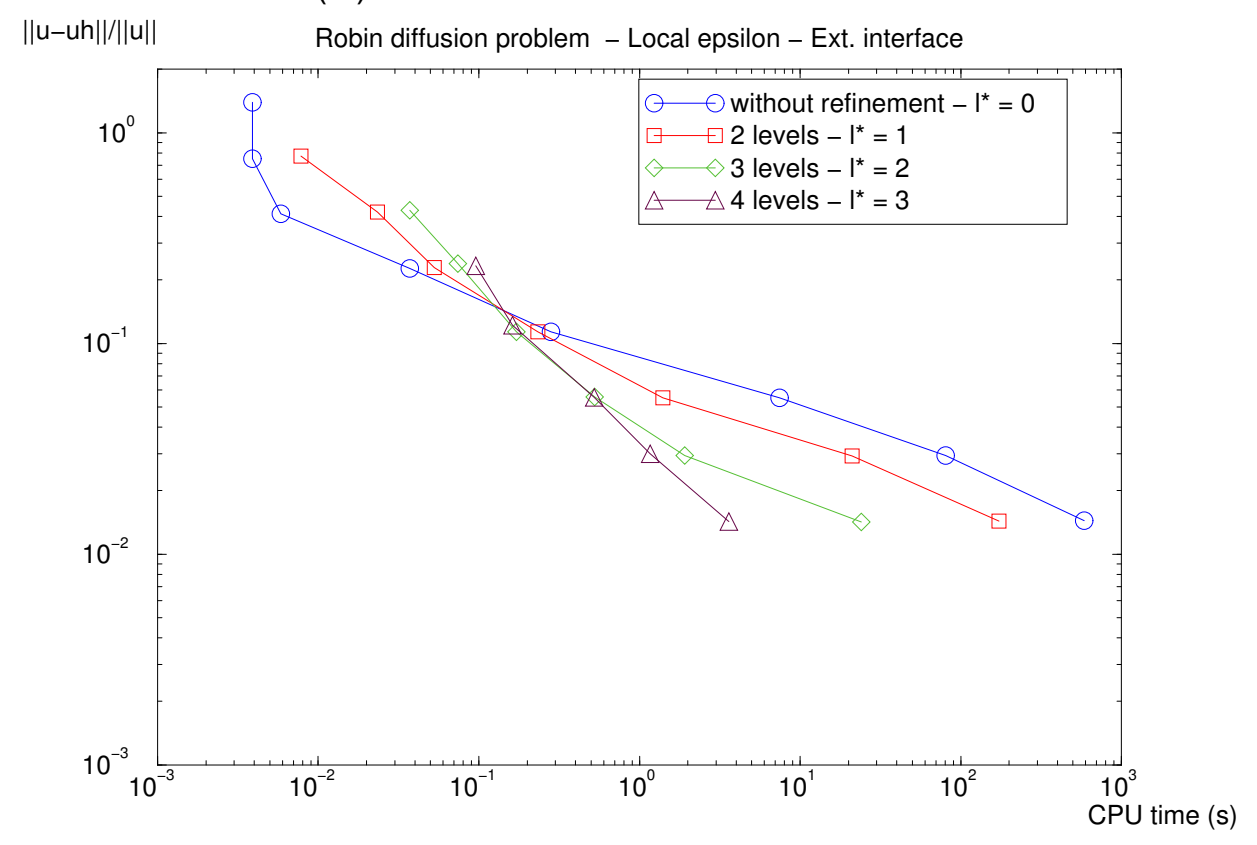

(b) Error versus CPU time.

Figure 21. Convergence for the $L^{2}$-norm of the discretization error for the combination of the $(R)$ E.B.C. method with the FIC-EBC solver without DDM iterations for the Robin diffusion problem with $\Sigma_{h}^{e x t}$. 
$\eta=10^{-12}$ to obtain a negligible modelling error compared to the discretization error. As in the diffusion case, the two Dirichlet E.B.C. methods are both first-order methods for the $L^{2}$-norm and lead to the same errors (see Fig. 22).

An adaptive mesh refinement is performed around the immersed interface for the $(D 1)$ E.B.C. method. As shown in Fig. 23, the (D1) E.B.C. method combined with the FIC-EBC algorithm is a first-order method for the $L^{2}$-norm. However for the cut interface with 4 levels $\left(l^{\star}=3\right)$, a stagnation appears: the error due to the non-refined zone of the original domain is reached. Hence, the combination of the E.B.C. method and the FIC-EBC solver varies like $\mathcal{O}\left(h_{l^{\star}}\right)$ until the discretization error of the non-refined zone is obtained.

If we look towards the error obtained for the $L^{\infty}$-norm, we can see in Fig. 24 that the behaviour of the max norm error is similar to the behaviour of the $L^{2}$-norm error. The E.B.C. method is of first-order for the $L^{\infty}$-norm, and the combination of the $(D 1)$ E.B.C. method with the FIC-EBC solver remains of first-order with respect to the finest local grid discretization step.

Moreover, the discretization of the convective flux makes the DDM iterations necessary even for the exterior interface $\Sigma_{h}^{e x t}$. The upstream scheme may require the unknown value of the neighbor control volume. If this control volume belongs to the enlargement zone of a patch and also belongs to the refinement zone of another patch, the values of the convective flux can then be really different. This may cause large differences on the next coarser grids.

\subsubsection{Robin problem}

We now consider the Robin problem

$$
\left\{\begin{aligned}
-\triangle \tilde{u}+\operatorname{div}(\tilde{\mathbf{v}} \tilde{u}) & =16 r^{2} & & \text { in } \tilde{\Omega} \\
\frac{\partial \tilde{u}}{\partial n} & =0 & & \text { on } \tilde{\Gamma} \\
-\frac{\partial \tilde{u}}{\partial n} & =\tilde{u}+3 & & \text { on } \Sigma\left(\alpha_{R}=1, g_{R}=3\right),
\end{aligned}\right.
$$

with $\tilde{\mathbf{v}}=2 r^{3} \mathbf{e}_{\mathbf{r}}$ where $r=\sqrt{x^{2}+y^{2}}$ and $\mathbf{e}_{\mathbf{r}}$ is the radial unit vector. The analytic solution of this problem is :

$$
\tilde{u}=2-\frac{5}{3} \exp \left(\frac{r^{4}-1}{2}\right) \quad \text { in } \tilde{\Omega} .
$$

Since $\left|\operatorname{meas}\left(\tilde{\Omega}_{h}\right)-\operatorname{meas}(\tilde{\Omega})\right|=\mathcal{O}(h)$, the expected accuracy is of first-order for the $L^{2}$-norm. In Fig. 25, the convergence of the $(R)$ E.B.C. method (see Table 1) for two kinds of surface correction is compared. A global approximation of the characteristic parameter $\epsilon_{h}$ (see Eq. (28)) leads to an asymptotic stagnation of the error and then the first-order precision is lost. With an accurate local correction (see Eq. (26)), the asymptotically first-order accuracy is then 


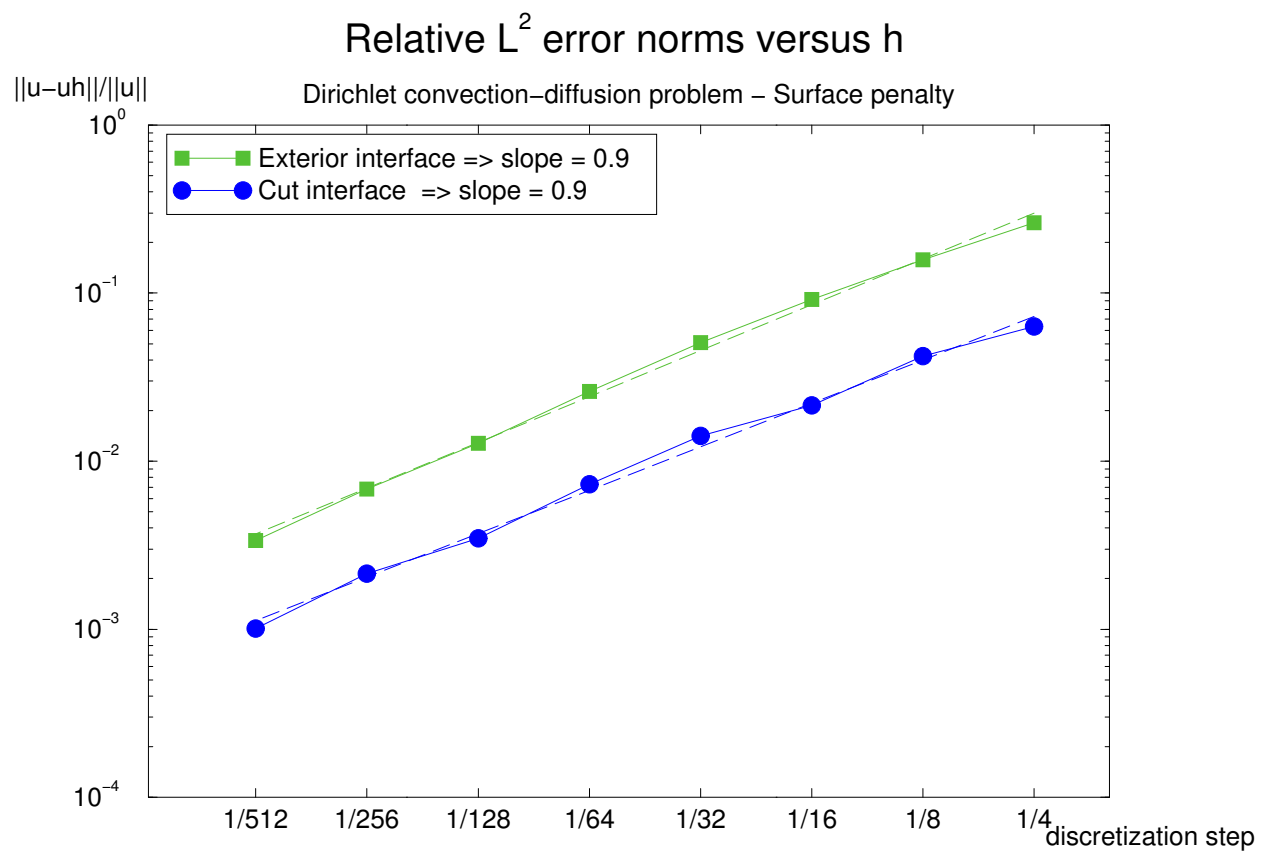

(a) (D1) E.B.C. method.

\section{Relative $L^{2}$ error norms versus $h$}

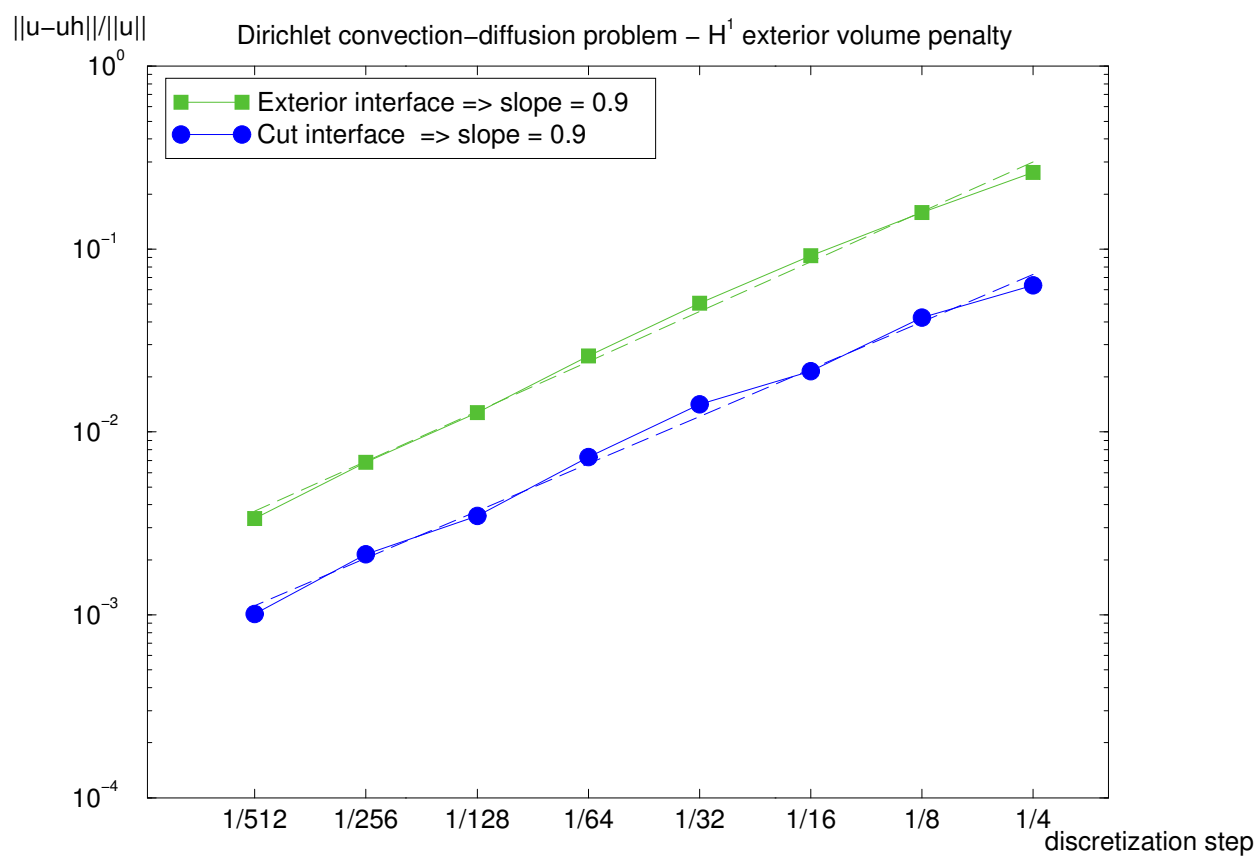

(b) (D2) E.B.C. method.

Figure 22. Convergence for the $L^{2}$-norm of the discretization error with $h$ for the Dirichlet convection-diffusion problem with $\Sigma_{h}^{e x t}$ or $\Sigma_{h}^{c u t}$. 
(D1) E.B.C. method + FIC-EBC solver + DDM

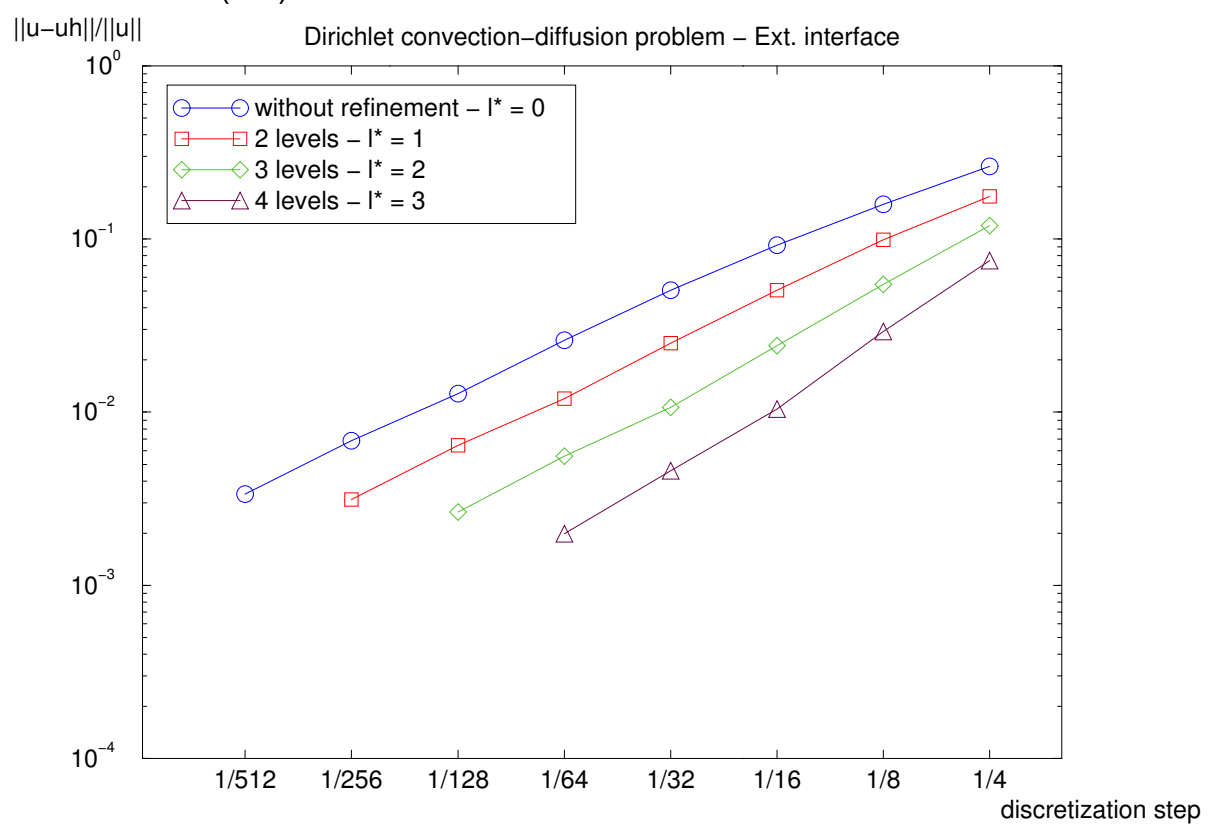

(a) Exterior interface $\Sigma_{h}^{e x t}$.

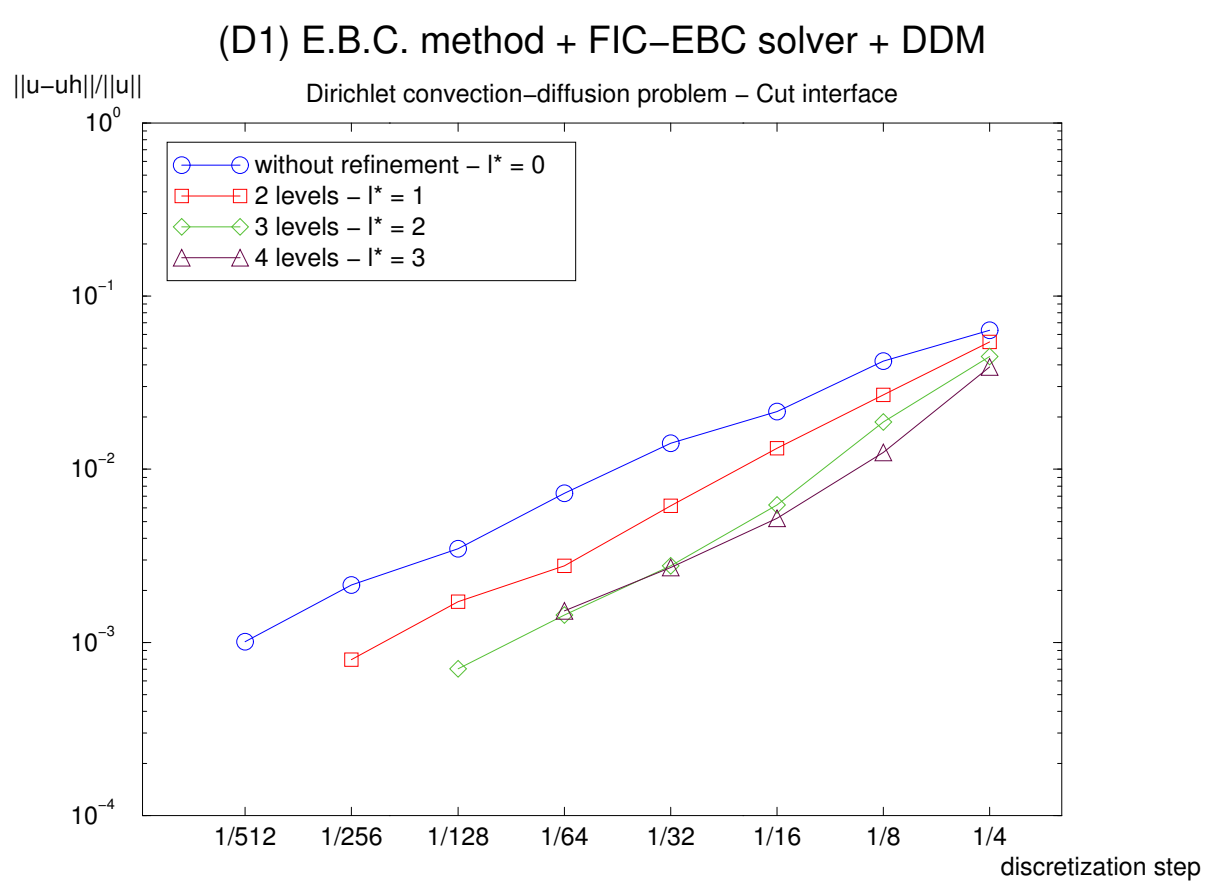

(b) Cut interface $\Sigma_{h}^{c u t}$.

Figure 23. Convergence for the $L^{2}$-norm of the discretization error with $h_{0}$ for the combination of the $(D 1)$ E.B.C. method with the FIC-EBC solver for the Dirichlet convection-diffusion problem. 
(D1) E.B.C. method + FIC-EBC solver + DDM

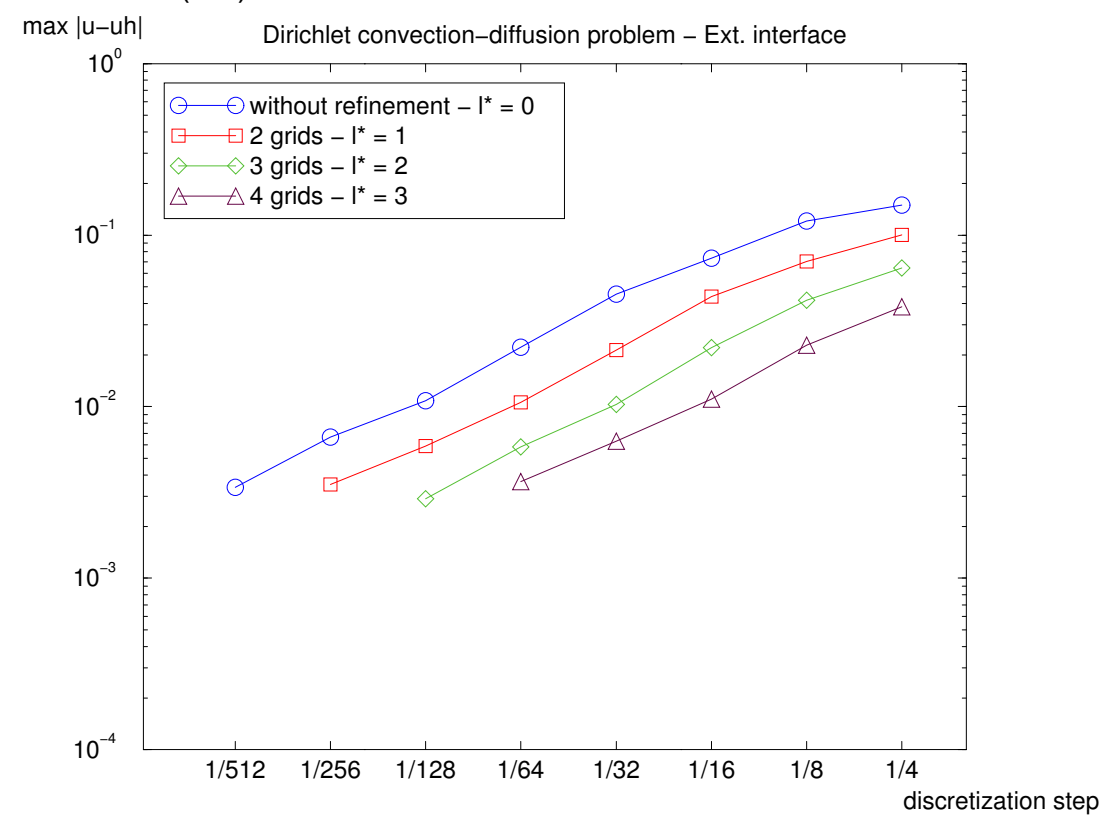

(a) Exterior interface $\Sigma_{h}^{e x t}$.

(D1) E.B.C. method + FIC-EBC solver + DDM

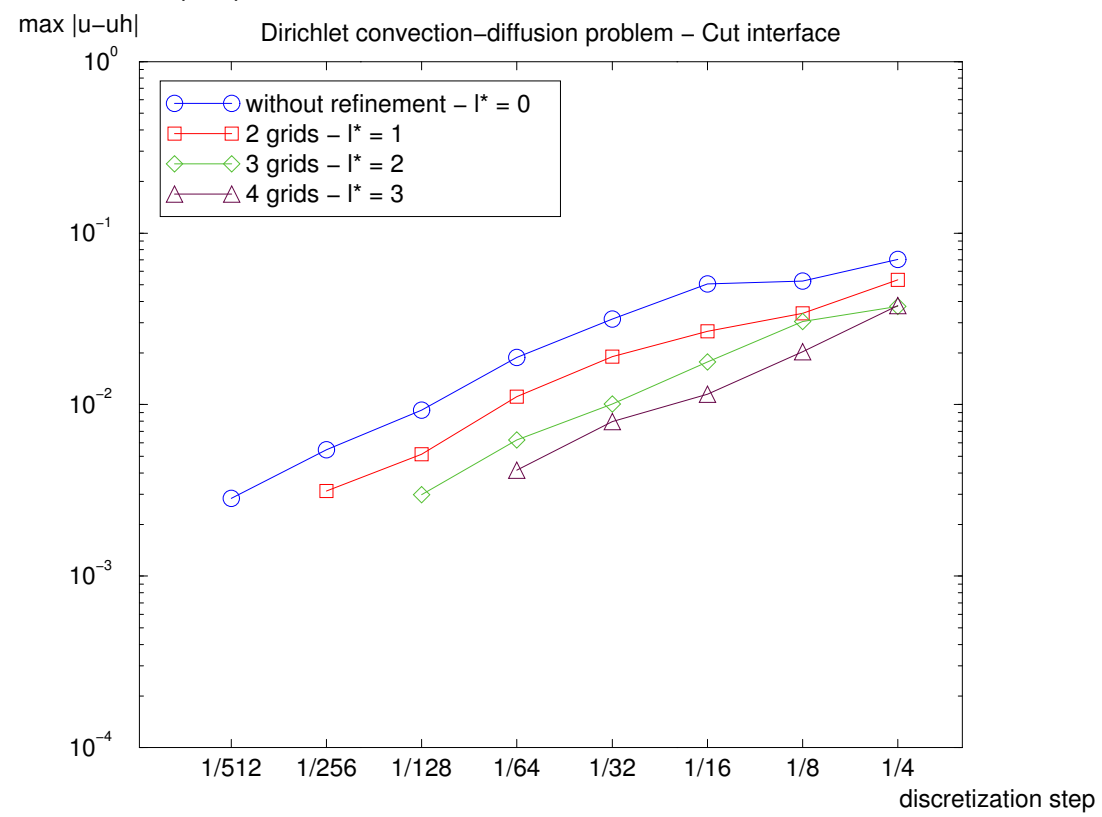

(b) Cut interface $\Sigma_{h}^{c u t}$.

Figure 24. Convergence for the $L^{\infty}$-norm of the discretization error with $h_{0}$ for the combination of the $(D 1)$ E.B.C. method with the FIC-EBC solver for the Dirichlet convection-diffusion problem. 
yielded for the $L^{2}$-norm error. Compared to diffusion problems, the asymptotic stagnation of the variant involving a global $\epsilon_{h}$ appears earlier, from the $8 \times 8$ mesh for the cut interface $\Sigma_{h}^{c u t}$. For Robin (or non homogeneous Neumann) problems, a local correction is thus required to keep the first-order method.

A multi-level algorithm with local nested patches around the immersed interface is performed with the method involving a local surface correction. As in the Dirichlet case, the FIC-EBC solver reduces the original coarse error until the stagnation due to the global error in the non-refined zone (see Fig. 26). Then, the combination of the E.B.C. method with the FIC-EBC solver leads to a convergence for the $L^{2}$-norm in $\mathcal{O}\left(h_{l^{\star}}\right)$ as long as the most important error remains around the immersed boundary.

The max norm convergence of the error obtained with the combination of $(R)$ E.B.C. method and the FIC-EBC solver is also of first order, see Fig. 27. For the cut approximate interface, the same stagnation as for the $L^{2}$-norm appears. The max norm error is then controlled be the max norm error of the non-refined zone.

\subsection{Second test problem: a corner domain}

We now consider an original polygonal domain $\tilde{\Omega}$ immersed in the unit square $\Omega=] 0,1[\times] 0,1\left[\right.$. That defines a corner immersed interface $\Sigma=\Sigma_{1} \cup \Sigma_{2}$, see Fig. 28(a). As in the quarter disk case (see section 5.3), the fictitious square domain is meshed with a grid step varying from $h=\frac{1}{4}$ to $h=\frac{1}{512}$. The approximate immersed interface $\Sigma_{h}$, lying on sides of the mesh, is chosen such that it crosses the physical immersed interface $\Sigma$, see Fig. 28(b). Then, the approximate physical domain verifies meas $\left(\left(\tilde{\Omega} \cup \tilde{\Omega}_{h}\right) \backslash\left(\tilde{\Omega} \cap \tilde{\Omega}_{h}\right)\right)=\mathcal{O}(h)$.

We want to solve the mixed problem

$$
(\tilde{\mathcal{P}}) \quad\left\{\begin{aligned}
-\triangle \tilde{u} & =4 & & \text { in } \tilde{\Omega}, \\
\frac{\partial \tilde{u}}{\partial n} & =0 & & \text { on } \tilde{\Gamma}, \\
\left.\tilde{u}\right|_{\Sigma_{1}} & =-\frac{4}{3} x^{2}+\frac{2}{\sqrt{3}} x+1 & & \text { on } \Sigma_{1}\left(u_{D}=-\frac{4}{3} x^{2}+\frac{2}{\sqrt{3}} x+1\right), \\
-\left.\frac{\partial \tilde{u}}{\partial n}\right|_{\Sigma_{2}} & =\left.\tilde{u}\right|_{\Sigma_{2}}+4 x^{2}-6 x+1+\sqrt{3} & & \text { on } \Sigma_{2}\left(\alpha_{R}=1, g_{R}=4 x^{2}-6 x+1+\sqrt{3}\right) .
\end{aligned}\right.
$$

with the analytic solution $\tilde{u}=2-\left(x^{2}+y^{2}\right)$ in $\tilde{\Omega}$.

This mixed problem is solved over the square domain $\Omega$ with the E.B.C. method $(D 1)$ on $\Sigma_{1, h}$ and the E.B.C. method $(R)$ on $\Sigma_{2, h}$ with a local correction parameter $\epsilon_{K}$. We combine these E.B.C. methods with the FIC-EBC 


\section{Relative $L^{2}$ error norms versus $h$}

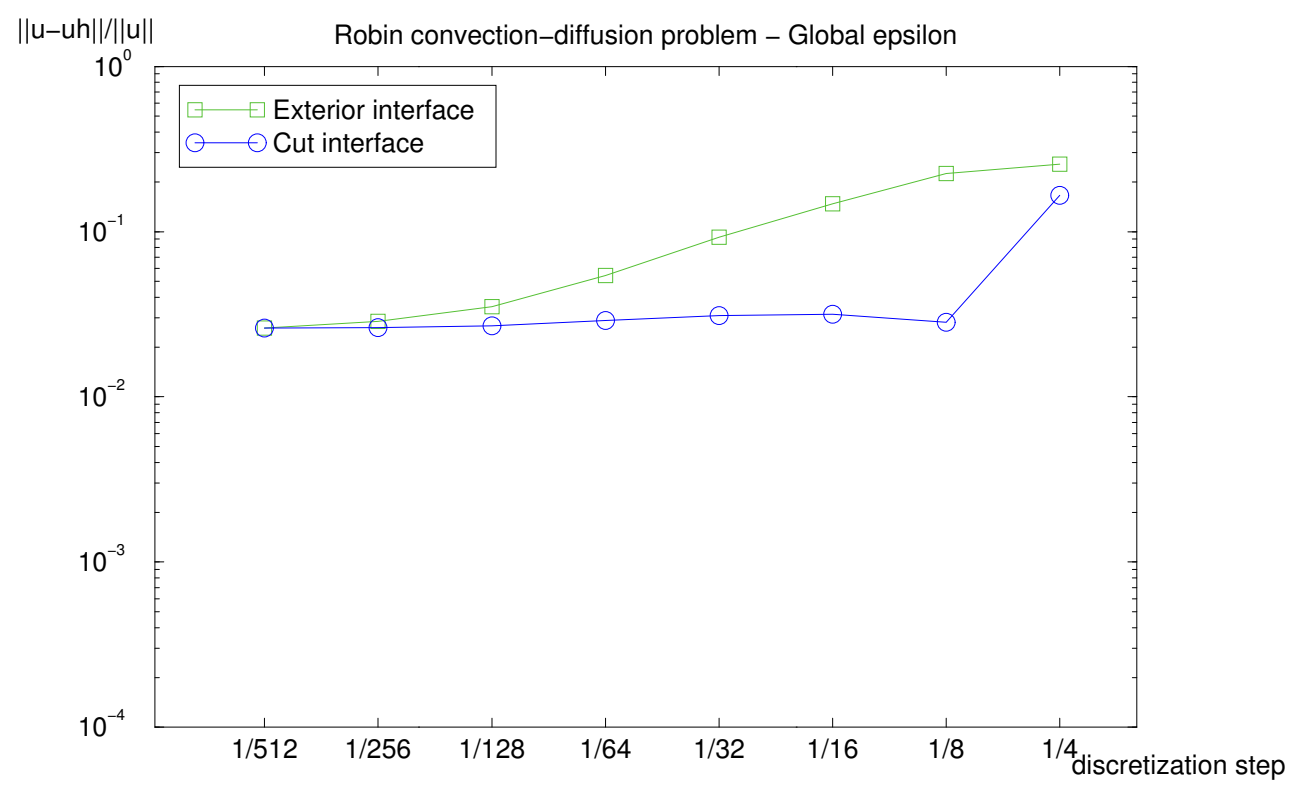

(a) Global correction with $\epsilon_{h}$.

\section{Relative $L^{2}$ error norms versus $h$}

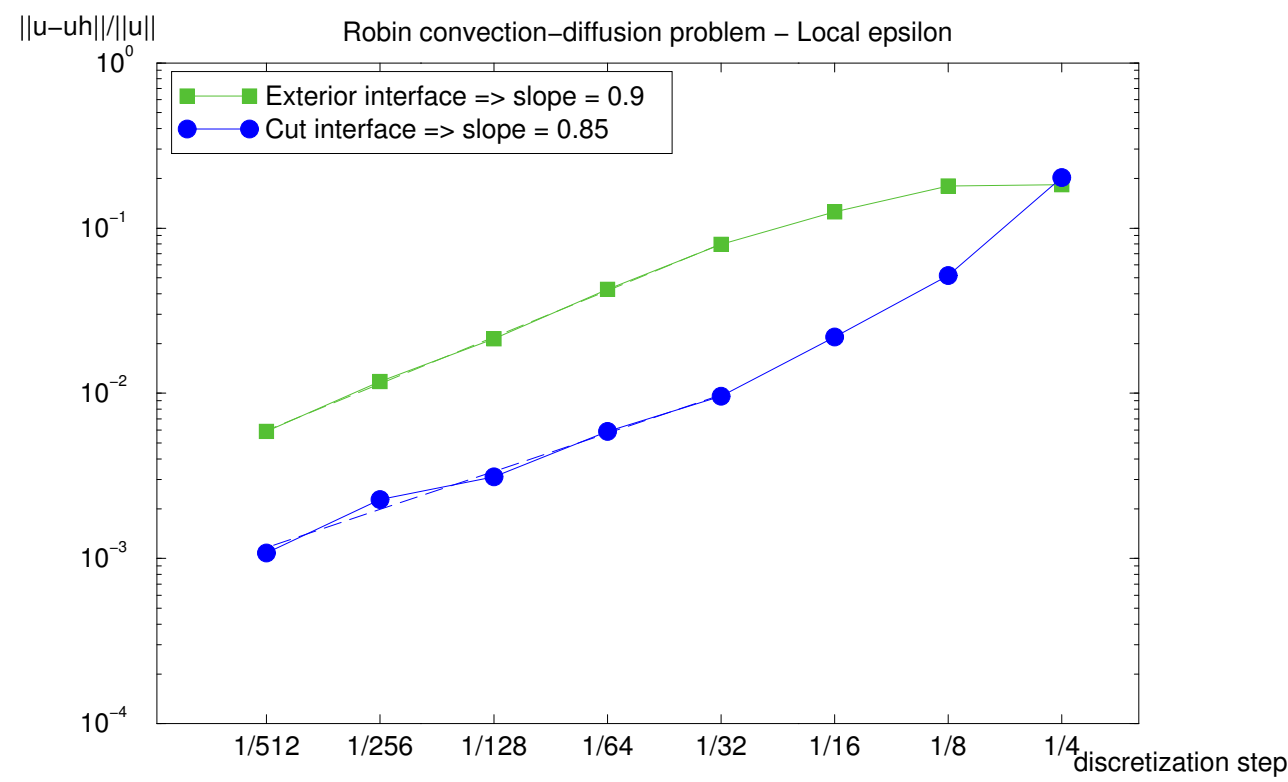

(b) Local correction $\epsilon_{K}$.

Figure 25. Convergence for the $L^{2}$-norm of the discretization error with $h$ of the $(R)$ E.B.C. method for the Robin convection-diffusion problem with $\Sigma_{h}^{e x t}$ or $\Sigma_{h}^{\text {cut }}$ : global correction and local correction with extension of the local surface $\Sigma_{l, K}$. 
(R) E.B.C. method + FIC-EBC solver + DDM

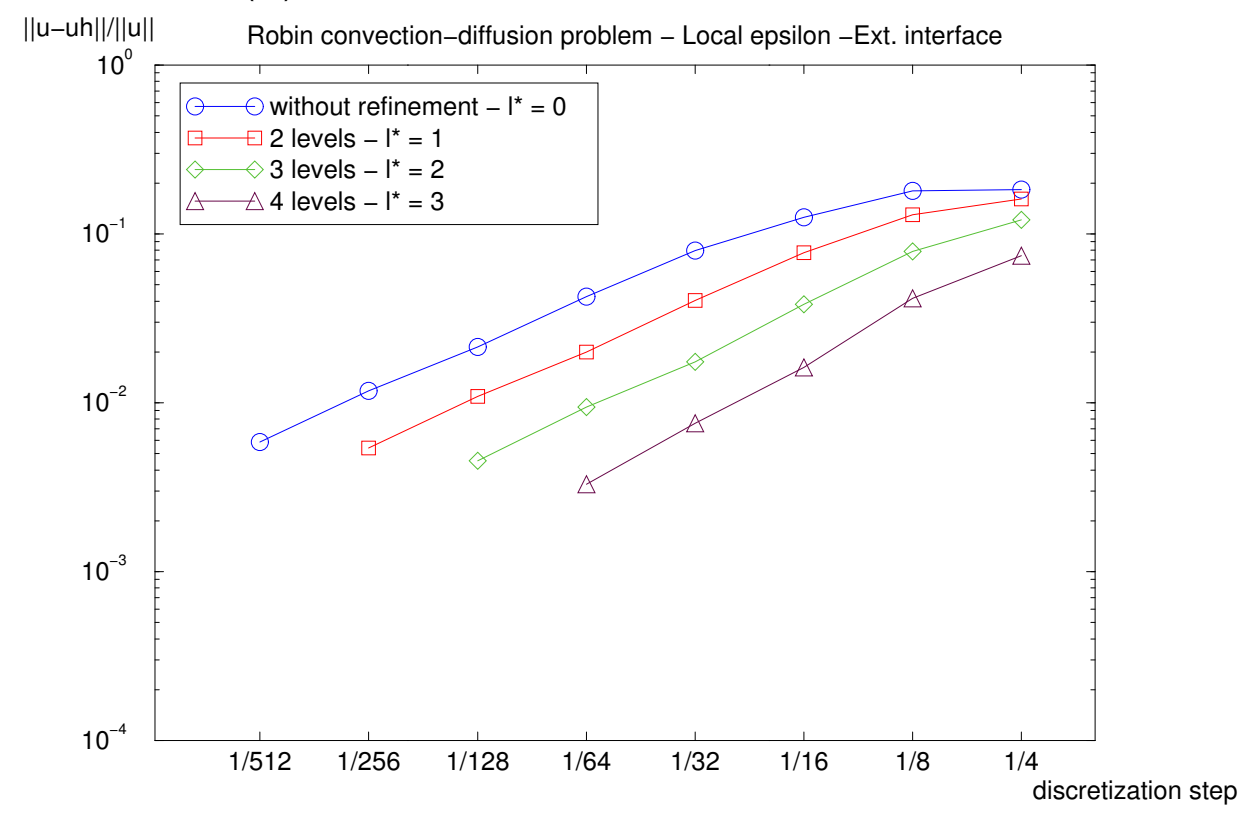

(a) Exterior interface $\Sigma_{h}^{e x t}$.

(R) E.B.C. method + FIC-EBC solver + DDM

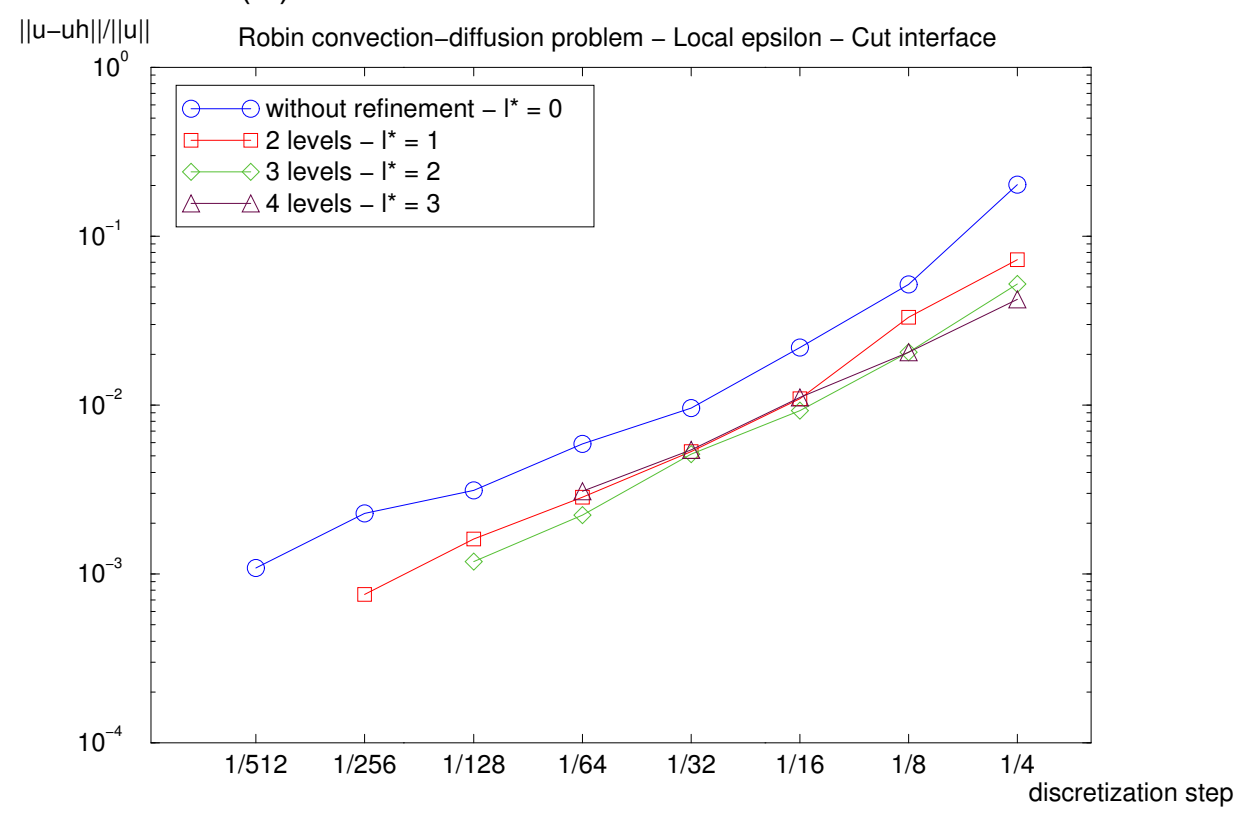

(b) Cut interface $\Sigma_{h}^{c u t}$.

Figure 26. Convergence for the $L^{2}$-norm of the discretization error with $h_{0}$ for the combination of the $(R)$ E.B.C. method with the FIC-EBC solver for the Robin convection-diffusion problem with a local correction $\epsilon_{K}$. 
(R) E.B.C. method + FIC-EBC solver + DDM

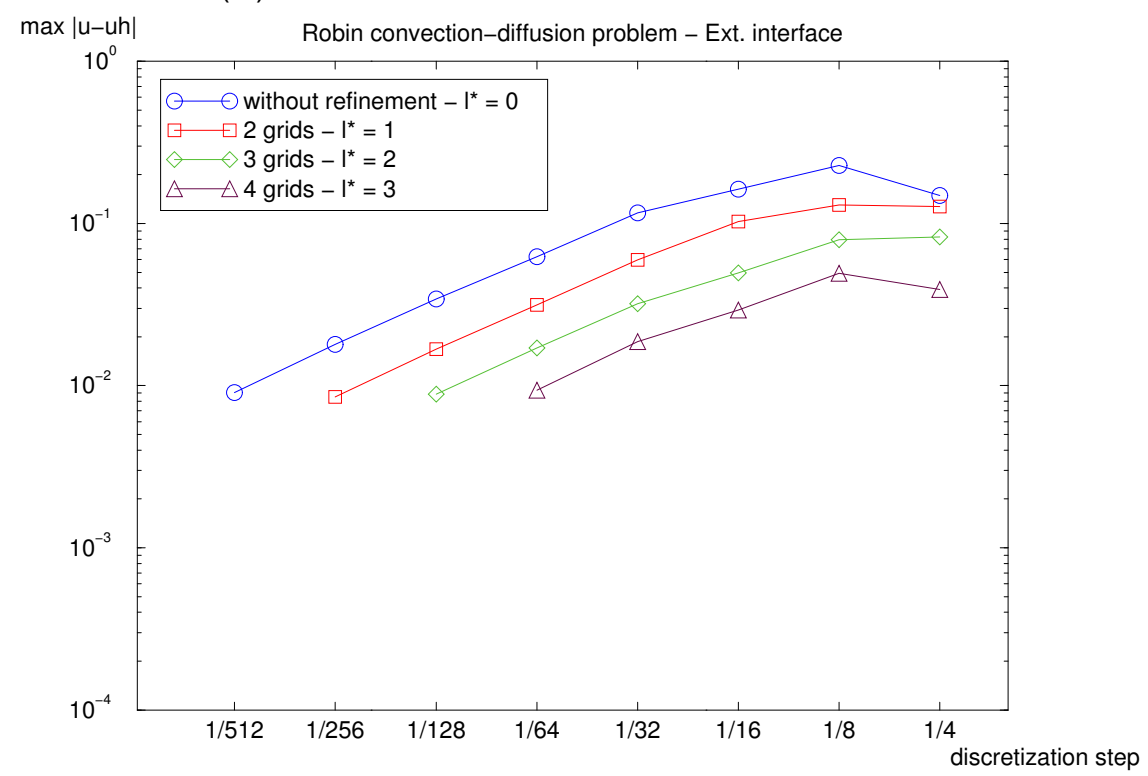

(a) Exterior interface $\Sigma_{h}^{e x t}$.

(R) E.B.C. method + FIC-EBC solver + DDM

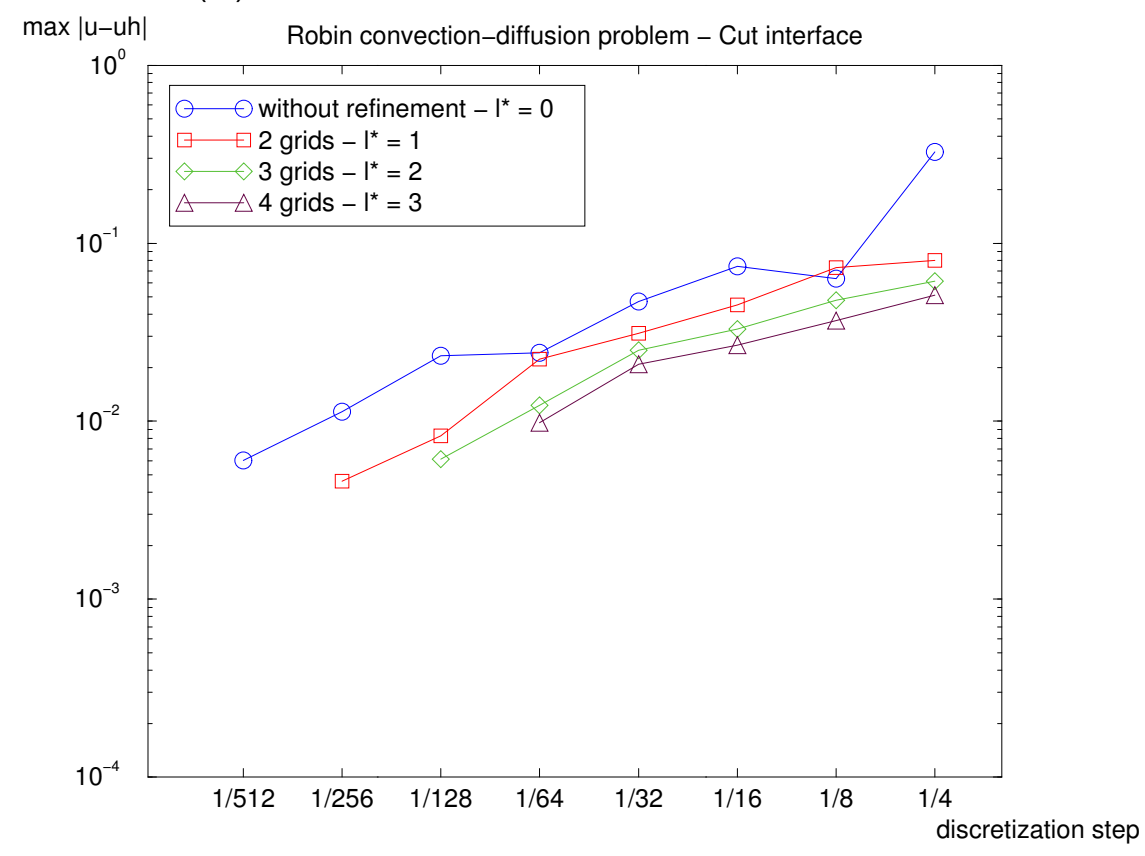

(b) Cut interface $\Sigma_{h}^{c u t}$.

Figure 27. Convergence for the $L^{\infty}$-norm of the discretization error with $h_{0}$ for the combination of the $(R)$ E.B.C. method with the FIC-EBC solver for the Robin convection-diffusion problem. 


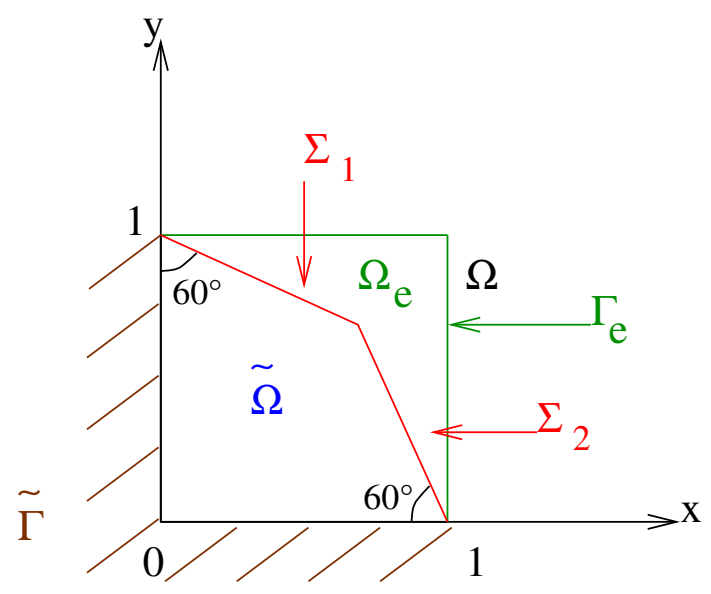

(a) Original corner domain and fictitious square domain

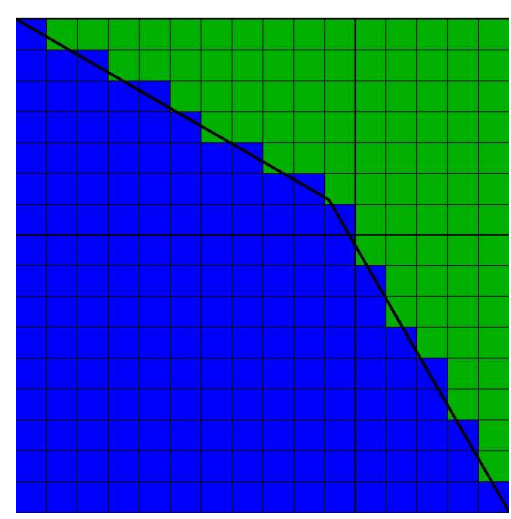

(b) Cut approximate immersed interface

Figure 28. Description of the corner domain, associated fictitious domain mesh and approximate immersed interface.

solver in order to improve the accuracy of the solution around the immersed interface. The results are reported in Fig. 29.

This test case enables us to conclude that even when the shape of the immersed interface is more singular (with corners for example), the E.B.C. method is in $\mathcal{O}(h)$ for the $L^{2}$-norm. Then, the combination with the FIC-EBC solver leads to a convergence in $\mathcal{O}\left(h_{l^{\star}}\right)$, with $l^{\star}$ the finest grid level, until the error of the non-refined zone is reached. Moreover, these results confirm that the fictitious domain method presented in this article can be used without difficulty for mixed boundary conditions. Indeed, for each general boundary condition, the same generic formulation with appropriate coefficients is used to evaluate the numerical fluxes.

\subsection{Analysis and comments on the numerical results}

In all the previous examples, the space discretization error is numerically measured by several manners. The $L^{2}$-norms (e.g. Fig. 19) or $L^{\infty}$-norms (e.g. Fig. 24) of the error inside the approximate original domain $\tilde{\Omega}_{h}$ are plotted with respect to the discretization step $h_{0}$ of the global coarse mesh. Typical distributions inside $\tilde{\Omega}_{h}$ of the absolute error calculated with or without local refinement are represented in Fig. 12. These numerical results enable us to draw the following conclusions for diffusion or convection-diffusion problems with Dirichlet, Robin or mixed embedded boundary conditions:

- for the $L^{2}$-norm and the $L^{\infty}$-norm, the mesh convergence of the E.B.C. methods asymptotically varies like $\mathcal{O}\left(h_{0}\right)$, where $h_{0}$ denotes the global mesh 


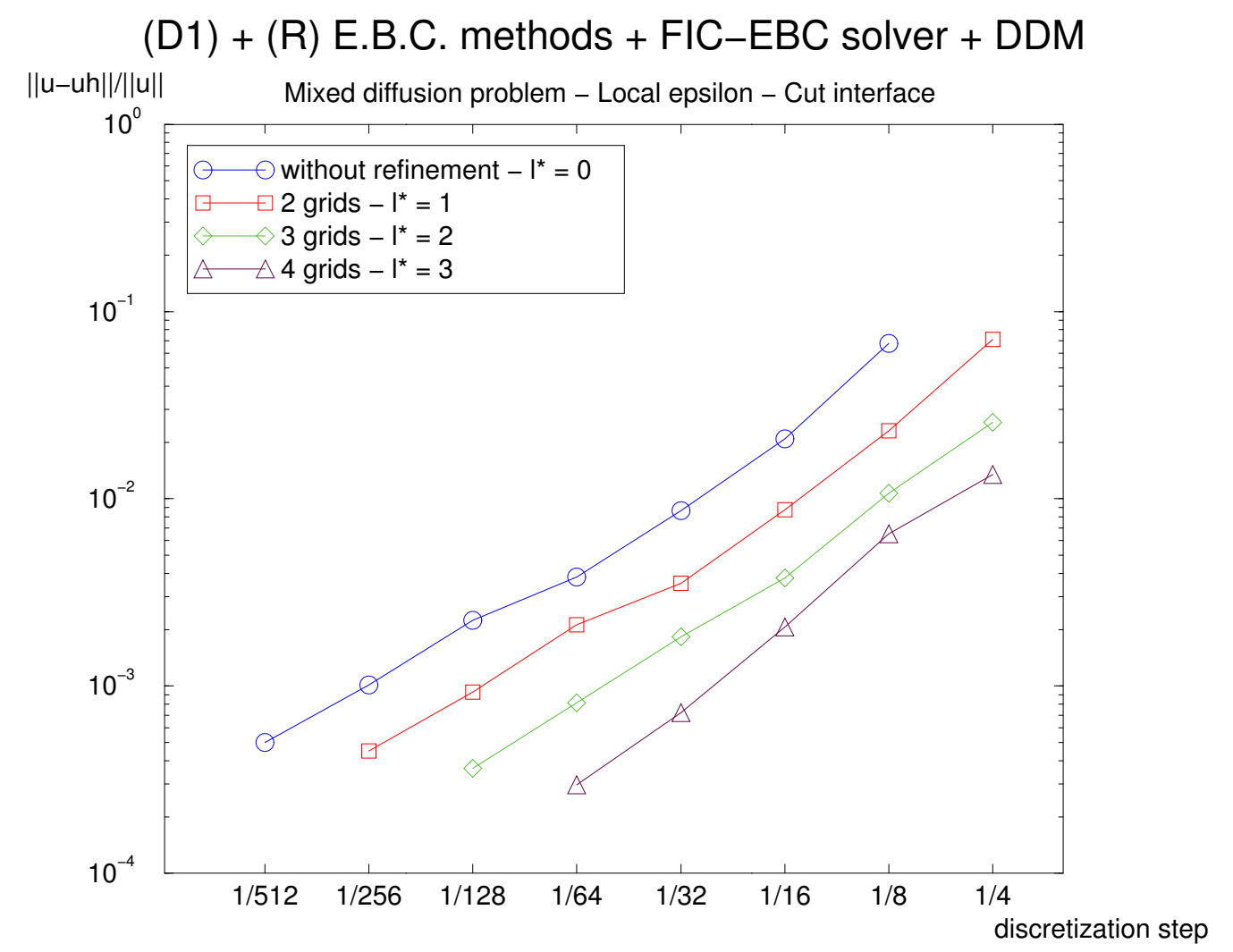

Figure 29. Convergence for the $L^{2}$-norm of the discretization error with $h_{0}$ for the combination of the $(D 1)$ and $(R)$ E.B.C. methods with the FIC-EBC solver for the mixed diffusion problem on the corner immersed domain.

step.

- when the multilevel FIC-EBC solver is used, the mesh convergence becomes in $\mathcal{O}\left(h_{l^{\star}}\right)$ where $h_{l^{\star}}$ denotes the discretization step of the local finest grid (last level of refinement). Indeed, the slope of all the plotted curves (in log$\log$ representation) is nearly one: the accuracy still varies as $\mathcal{O}\left(h_{0}\right)$. Moreover, the error obtained with $l^{\star}$ refinement levels from a given global mesh step $h_{0}$ is reduced to the error obtained without refinement (monogrid calculation) with a global mesh step equal to $h_{l^{\star}}$. However the $\mathcal{O}\left(h_{l^{\star}}\right)$ convergence holds until a maximum number of refinement levels is reached, over which no more improvement of the discretization error is observed (see Fig. 23 and Fig. 26). Then, only the $\mathcal{O}\left(h_{0}\right)$ convergence remains.

- the stagnation of the error, which can be observed when the number of refinement levels increases, is due to the residual discretization error in the non-refined zone of the global mesh. This residual error is also of firstorder. Indeed, as we solve elliptic problems, the influence of the boundary conditions is visible on the whole domain and consequently remains non negligible far away from the boundary. The error distribution inside the approximate original domain confirms this conclusion, see Fig. 12. Thus, the discretization error of non-refined zone (which does not contain the immersed boundary) is always spoiled by the poor discretization of the 
immersed interface by stair cases, even with a local mesh refinement around this immersed boundary. Whatever the number of refinement levels $l^{\star}$ is, the residual error converges like $\mathcal{O}\left(h_{0}\right)$ and never like $\mathcal{O}\left(h_{0}^{2}\right)$, which is clearly observed in the mesh convergence results.

These previous remarks suggest that the discretization error in the approximate original domain $\tilde{\Omega}_{h}$ converges for the $L^{2}$-norm and by the same way for the $L^{\infty}$ norm with the following law

$$
e_{r}\left(u_{h}\right) \simeq c_{0} h_{0}+C_{l^{\star}} h_{l^{\star}}, \quad 0<c_{0}<C_{0}<C_{1}<\cdots<C_{l^{\star}}, \quad h_{l^{\star}}=\frac{h_{0}}{2^{l^{\star}}},
$$

where the constants $c_{0}, C_{l}, 0 \leqslant l \leqslant l^{\star}$ do not depend on the discretization steps. Thus, the term $c_{0} h_{0}$ is the residual stagnation error in the non-refined zone. This numerical estimation summarizes the previous conclusions:

- without refinement $\left(l^{\star}=0\right)$, the first order accuracy with respect to $h_{0}$ is obtained

$$
e_{r}\left(u_{h}\right) \simeq\left(c_{0}+C_{0}\right) h_{0} .
$$

- while $l^{\star}$ is sufficiently small in order to have

$$
c_{0} h_{0} \ll C_{l^{\star}} h_{l^{\star}},
$$

the mesh convergence varies like $\mathcal{O}\left(h_{l^{\star}}\right)$.

- as soon as the number of refinement levels $l^{\star}$ is bigger than a "stagnation" number of refinement levels $l_{\text {stag }}^{\star}$, the residual stagnation error is reached. So we have

$$
C_{l^{\star}} h_{l^{\star}} \ll c_{0} h_{0}, \quad l^{\star} \geqslant l_{\text {stag }}^{\star},
$$

where

$$
C_{l_{\text {stag }}^{\star}} h_{l_{\text {stag }}^{\star}} \simeq \nu c_{0} h_{0}, \quad \nu \ll 1 .
$$

Then, for $l^{\star}$ bigger than $l_{\text {stag }}^{\star}=\frac{\ln \left(\frac{C_{l_{\text {stag }}^{\star}}}{\nu c_{0}}\right)}{\ln 2}$, which is a bounded number of refinement levels independent of $h_{0}$, the global error $e_{r}\left(u_{h}\right)$ is equal to the residual error $c_{0} h_{0}$. The correction of the solution around the immersed interface is then negligible compared to the residual error of the non-refined part.

The figures of the discretization error versus the CPU time (Figs. 14 and 20) and corresponding tables with respect to $h_{0}$ and $l^{\star}$ (Tables 2 and 4 ) show the performances of the multilevel solver.

In view of all the previous comments, the optimal strategy to obtain a given error is first to increase the number of refinement levels $l^{\star}$ before to decrease $h_{0}$ if the residual stagnation error is reached.

The resolution of 3-D problems with the previous E.B.C. methods combined 
with the FIC-EBC solver should follow the same strategy. In particular, the mesh convergence will be of first-order: $\mathcal{O}\left(h_{l^{\star}}\right)$ or $\mathcal{O}\left(h_{0}\right)$ as in 2-D. There still exists a maximum number of refinement levels independent of $h_{0}$ over which no improvement of the solution is observed. However, we may expect that the multilevel solver would be relatively less efficient than in 2-D. In this case, the improvement of the ratio between the solution precision and the CPU time will be surely a little bit less important.

\section{Conclusion and perspectives}

An accurate fictitious domain approach to solve elliptic problems with general boundary conditions has been introduced. This method is based on the introduction of embedded transmission conditions linking jumps of flux and solution through the immersed interface. This method is low cost since a unique Cartesian mesh of the fictitious domain is required. As this method yields a first-order convergence for the $L^{2}$ and $L^{\infty}$-error norm, an adaptive refinement algorithm is implemented to improve the accuracy of the solution. This algorithm takes account of the properties of the fictitious domain method introduced here and then an asymptotic convergence in $\mathcal{O}\left(h_{l^{\star}}\right), h_{l^{\star}}$ being the discretization step of the finest local grid, can be observed until the discretization error of the non-refined zone is reached. The numerical results obtained for Dirichlet, Robin or mixed problems are very satisfactory.

Such a fictitious domain method is full of promise, especially to simulate moving and deformable boundaries with a cheap computational cost since no boundary-fitted remeshing is required. Only the local patches of refinement have to be adjusted around the moving immersed boundary. The generalization of this embedded boundary conditions method to the corresponding parabolic evolution problems is also straightforward. The next step will consist in extending this fictitious domain method to the resolution of Navier-Stokes equations (with a projection step for example) with moving boundaries problems like two phase flow simulations or fluid/structure interactions (see [45]).

\section{Acknowledgments}

The authors would like to thank $\mathrm{H}$. Lomenède for his contribution to the development of the FV code. The authors acknowledge the support from the French CEA/EDF Neptune Project. 


\section{References}

[1] V.K. Saul'ev. On the solution of some boundary value problems on high performance computers by fictitious domain method. Siberian Math. Journal, 4(4):912-925 (in Russian), 1963.

[2] G.I. Marchuk. Methods of Numerical Mathematics. Application of Math. 2, Springer-Verlag New York (1rst ed. 1975), 1982.

[3] Ph. Angot. A model of fracture for elliptic problems with flux and solution jumps. C.R. Acad. Sci. Paris, Ser I Math., 337 (6) 425-430, 2003.

[4] Ph. Angot. A unified fictitious domain model for general embedded boundary conditions. C.R. Acad. Sci. Paris, Ser. Math., Vol. 341(11), pp 683-688, 2005.

[5] W. Hackbusch. Multigrid Methods and applications. Series in computer mathematics, Springer-Verlag, 1985.

[6] K. Khadra, Ph. Angot, J.-P. Caltagirone, and P. Morel. Concept de zoom adaptatif en architecture multigrille locale; étude comparative des méthodes L.D.C., F.A.C. et F.I.C. Mathematical Modelling and Numerical Analysis, 30(1):39-82, 1996.

[7] X. Coré, Ph. Angot, and J.-C. Latché. A multilevel local mesh refinement projection method for low mach number flows. Mathematics and Computers in Simulation - IMACS Int. J., Vol. 61, no 3-6, pp. 477-488, 2003.

[8] K. Khadra, Ph. Angot, S. Parneix, and J.-P. Caltagirone. Fictitious domain approach for numerical modelling of Navier-Stokes equations. Int. J. Numer. Meth. in Fluids, Vol. 34(8): 651-684, 2000.

[9] R. Glowinski, T.-W. Pan, and J. Périaux. A fictitious domain method for Dirichlet problem and applications. Comp. Meth. in Appl. Mech. and Eng., 111 (3-4) : 283-303, 1994.

[10] R.J. Leveque and Z. Li. The immersed interface method for elliptic equations with discontinuous coefficients and singular sources. SIAM J. Numer. Anal., 31:1019-1044, 1994.

[11] F. Collino, P. Joly, and F. Millot. Fictitious domain method for unsteady problems. J. of Comp. Physics, Volume 138, Issue 2, pp. 907-938, 1997.

[12] Ph. Angot. Finite volume methods for non smooth solution of diffusion models; Application to imperfect contact problems. Recent Advances in Numerical Methods and Applications-Proceedings 4th Int. Conf. NMA'98, Sofia (Bulgaria) 19-23 August 1998, pp.621-629, World Scientific Publishing, 1999.

[13] Ph. Angot. Analysis of singular perturbations on the Brinkman problem for fictitious domain models of viscous flows. $M^{2} A S$ Math. Meth. in the Appl. Sci., Vol. 22(16), pp. 1395-1412, 1999. 
[14] P. McCorquodale, P. Colella, and H. Johansen. A Cartesian grid embedded boundary method for the heat equation on irregular domains. J. Comput. Phys., 173:620-635, 2001.

[15] P.W. Hemker, W. Hoffmann, and M.H. van Raalte. Discontinuous Galerkin discretization with embedded boundary conditions. Comput. Meth. Appl. Math., Vol. 3(1), pp. 135-158, 2003.

[16] R. Glowinski, T.-W. Pan, Jr.R. Wells, and X. Zhou. Wavelet and finite element solutions for the Neumann problem using fictitious domains. Journal Comput. Phys., Vol. 126(1),pp.40-51, 1996.

[17] P. Joly and L. Rhaouti. Fictitious domains, H(div) finite elements and Neumann condition: the inf-sup condition. C. R. Acad. Sci. Paris, Ser. I Math., Vol. 328(12), pp. 1225-1230, 1999.

[18] A.L. Fogelson and J.P. Keener. Immersed interface methods for Neumann and related problems in two and three dimensions. SIAM J. Sci. Comput., Vol 22, pp. 1630-1684, 2000.

[19] M.S. Mommer. A smoothness preserving fictitious domain method for elliptic boundary-value problems. IMA J. of Numer. Anal., doi:10.1093/imanum/dri045, 2005.

[20] P. Schwartz, M. Barad, Ph. Colella, and T. Ligocki. A Cartesian grid embedded boundary method for the heat equation and Poisson's equation in three dimensions. J. Comput. Phys., 211:531-550, 2006.

[21] V.D. Kopčenov. A method of fictitious domains for the second and third value problems. Trudy Mat. Inst. Steklov, Vol 131, pp.119-127 (in Russian), 1974.

[22] S. Del Pino and O. Pironneau. A fictitious domain based on general PDE's solvers. Proc. ECCOMAS 2001, Sept Swansea. K. Morgan ed. Wiley., 2002.

[23] Ph. Angot, H. Lomenède, and I. Ramière. A general fictitious domain method with non-conforming structured meshes. Finite Volume for Complex Applications IV, Problems and Perspectives, Marrakech, Ed. F. Benkhaldoun, D. Ouazar, S. Raghay - Hermes Science, pp. 261-272, 2005.

[24] I. Ramière, Ph. Angot, and M. Belliard. Fictitious domain methods to solve convection-diffusion problems with general boundary conditions. AIAA 2005-4709. 17th Computational Fluid Dynamics Conference - AIAA. Toronto, Canada, 6-9 June, 2005.

[25] R. Glowinski and Y. Kuznetsov. On the solution of the Dirichlet problem for linear elliptic operators by a distributed Lagrange multiplier method. C.R. Acad. Sci. Paris, t.327, Serie I, pp. 693-698, 1998.

[26] J.-F. Maitre and L. Tomas. A fictitious domain method for Dirichlet problems using mixed finite elements. Appl. Math. Let., Vol. 12(4), pp. 117-120, 1999.

[27] H. Johansen and Ph. Colella. A Cartesian grid embedded boundary method for Poisson's Equation on irregular domains. J. Comput. Phys., 14\%:60-85, 1998. 
[28] P. McCorquodale, Ph. Colella, D.P. Grote, and J.-L. Vay. A node-centered local refinement algorithm for Poisson's equation in complex geometries. J. Comput. Phys., 201:34-60, 2004.

[29] Z. Li. An overview of the immersed interface method and its applications. Taïwan, J. Math., 7:1-49, 2003.

[30] C.S. Peskin. The immersed boundary method. Acta Numerica, Vol 11, pp 479-517, 2002.

[31] S. Bertoluzza, M. Ismail, and B. Maury. The FBM method: Semi-discrete scheme and some numerical experiments. Lecture Notes in Comp. Sc. and Eng., 2004.

[32] I. Ramière, Ph. Angot, and M. Belliard. A fictitious domain approach with spread interface for elliptic problems with general boundary conditions. Comp. Meth. in Appl. Mech. and Eng., vol. 196 (4-6), pp.766-781, 2007.

[33] L.A. Rukhovets. A remark on the method of fictive domains. Differential Equations,3,4 (in Russian), 1967.

[34] S. Turek, D.C. Wan, and L.S. Rivkind. The Fictitious Boundary Method for the implicit treatment of Dirichlet boundary conditions with applications to incompressible flow simulations. Lecture Notes in Computational Science and Engineering, Volume 35, Springer Verlag, 2003.

[35] D. Wan and S. Turek. Direct numerical simulation of particulate flow via multigrid FEM techniques and the fictitious boundary method. Int. J. Numer. Meth. Fluids, Vol. 51, pp. 531-566, 2006.

[36] Ph. Angot, Ch.-H. Bruneau, and P. Fabrie. A penalization method to take into account obstacles in incompressible viscous flows. Nümerische Mathematik, 81(4):497-520, 1999.

[37] R. Eymard, Th. Gallouët, and R. Herbin. Finite Volume methods. P.G. Ciarlet, J.-L. Lions (Eds), Handbook of Numerical Analysis, vol. VII, North-Holland, pp.713-1020, 2000.

[38] Ph. Angot and I. Ramière. Convergence analysis of the $Q_{1}$-finite element method for elliptic problems with non-boundary fitted meshes. IMA J. of Numer. Analysis, submitted, 2006.

[39] V. Girault and R. Glowinski. Error analysis of a fictitious domain method applied to a Dirichlet problem. Japan Journal of Industrial and Applied Mathematics, vol. 12(3), 487-514, 1995.

[40] Ph. Angot, J.P. Caltagirone, and K. Khadra. Une méthode adaptative de raffinement local : la Correction du Flux à l'Interface. C.R. Acad. Sci. Paris, 315 (1), pp. 739-745, 1992.

[41] Ph. Angot and M. Laugier. La méthode FIC de raccordement conservatif de sous-domaines emboîtés pour un modèle de circulation océanique. C.R. Acad. Sci. Paris, 319 (2), pp. 993-1000, 1994. 
[42] P. Le Tallec. Domain decomposition methods in computational mechanics. Computational Mechanics advances, Vol. 1, North Holland, Elsevier Science, pp. 121-220, 1994.

[43] M.R. Hestenes and E.L. Stiefel. Methods of conjugate gradients for solving linear systems. J. Res. Nat. Bur. Standards Sect. 5, vol. 49, pp. 409-436, 1952.

[44] V.A. Van Der Vost. Bi-CGSTAB: A fast and smoothly converging variant of Bi-CG for the solution of nonsymmetric linear systems. SIAM J. Sci. Stat. Comput. 13, 631-644, 1992.

[45] Ph. Angot. A fictitious domain model for the Stokes/Brinkman problem. C.R. Acad. Sci. Paris, Ser. Math., accepted, 2006. 